\title{
Investigation of Mechanical Differentials as Continuously Variable Transmissions
}

Dax B. Wells

Brigham Young University - Provo

Follow this and additional works at: https://scholarsarchive.byu.edu/etd

Part of the Mechanical Engineering Commons

\section{BYU ScholarsArchive Citation}

Wells, Dax B., "Investigation of Mechanical Differentials as Continuously Variable Transmissions" (2010). Theses and Dissertations. 2904.

https://scholarsarchive.byu.edu/etd/2904

This Thesis is brought to you for free and open access by BYU ScholarsArchive. It has been accepted for inclusion in Theses and Dissertations by an authorized administrator of BYU ScholarsArchive. For more information, please contact scholarsarchive@byu.edu, ellen_amatangelo@byu.edu. 
Investigation of Mechanical Differentials as

Continuously Variable Transmissions

Dax B. Wells

A thesis submitted to the faculty of

Brigham Young University

in partial fulfillment of the requirements for the degree of

Master of Science

\author{
Robert H. Todd, Chair \\ Perry W. Carter \\ Kenneth W. Chase
}

Department of Mechanical Engineering

Brigham Young University

December 2010

Copyright @ 2010 Dax B. Wells

All Rights Reserved 



\author{
ABSTRACT \\ Investigation of Mechanical Differentials as \\ Continuously Variable Transmissions
}

Dax B. Wells

Department of Mechanical Engineering

Master of Science

In recent years the increasing demand for fuel efficient and less pollutant vehicles has stimulated the development of hybrid and electric vehicles. These vehicle platforms often incorporate drivetrains which utilize multiple power sources for vehicle propulsion in an effort to increase fuel mileage and reduce emissions. Coupling multiple power sources, such as an internal combustion engine and electric motor(s), has new challenges in drivetrain design. Understanding the torque and rpm relationships within the power transmission device used to combine power sources is fundamental to overcoming the design challenges associated with hybrid and electric vehicle platforms.

Results from this research include the fundamental torque and rpm relationships that exist in a multiple-input, single-output power transmission device. These results were deduced from a test that incorporated two separate power inputs into a differential which combined to produce a single output. Testing displayed that a differential has the ability to function as an infinitely variable transmission (IVT). Additionally, the challenges associated with using a differential as a multiple-input, single-output device were identified. Recommendations for overcoming these challenges are also presented herein. This work provides the basis for future work in powertrain optimization for multiple-input, single-output transmission devices.

Keywords: Dax Wells, PECVT, PEIVT, continuously variable transmission, positively engaged continuously variable transmission, infinitely variable transmission, positively engaged infinitely variable transmission, differential 



\section{ACKNOWLEDGMENTS}

I wish to acknowledge the help and guidance of my Heavenly Father. I express my gratitude to Him for the insight He has imparted to myself and others, and for those He prepared and placed in my path to mentor, support, and strengthen me throughout the course of this research.

I thank Brigham Young University and the Department of Mechanical Engineering for the facilities and resources they have provided during this research.

I express my appreciation to my graduate advisor, Dr. Robert Todd of the Department of Mechanical Engineering for his kindness, guidance, and enthusiasm toward me as an individual. The opportunity to work closely with Dr. Todd has helped me become a better person and a more competent engineer.

I thank Dr. Kenneth Chase of the Department of Mechanical Engineering and Dr. Perry Carter from the School of Technology in the Manufacturing Engineering \& Technology Program for their mentorship and feedback on my work.

I acknowledge the help of Kevin Cole, Ken Forester, and Scott Daniels who have provided technical support for sourcing and implementing hardware and software.

I also express my appreciation for my fellow graduate students in the Compliant Mechanisms Research Lab, for their insight and thoughts throughout the course of my research. Specifically, I wish to express gratitude to my associates Benjamin Groen and John Wyall who continuously provided support, encouragement, and expertise throughout my research.

Lastly, I wish to express my gratitude and love for my dear wife Jennifer. She has been a source of encouragement and strength. 



\section{TABLE OF CONTENTS}

LIST OF TABLES $\ldots \ldots \ldots \ldots \ldots \ldots \ldots \ldots \ldots$ viii

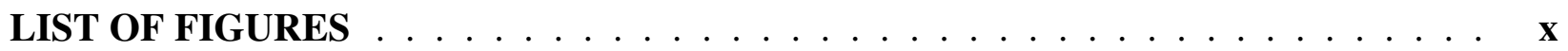

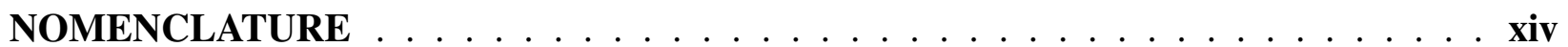

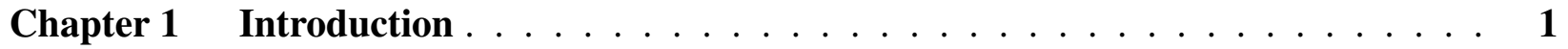

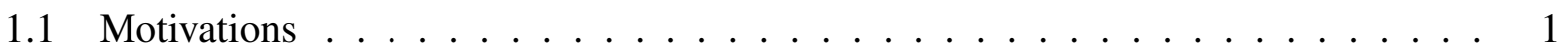

1.2 Research Objectives . . . . . . . . . . . . . . . . . . . 6

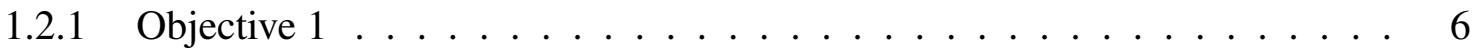

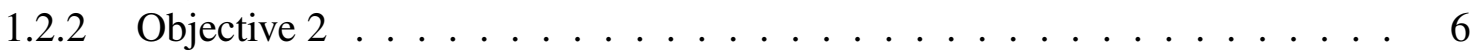

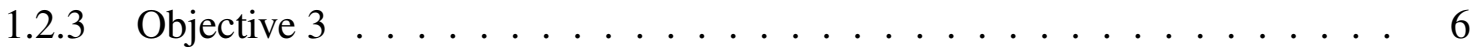

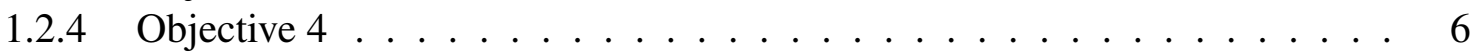

Chapter 2 Background . . . . . . . . . . . . . . . . . . . . . 9

2.1 Function Of A Mechanical Differential . . . . . . . . . . . . . . . . . . . . 9

2.2 Variable Transmissions . . . . . . . . . . . . . . . . . . . . . . . 11

2.3 Hybrid Powertrains . . . . . . . . . . . . . . . . . . . . . . . . . . 14

2.3.1 Toyota Prius . . . . . . . . . . . . . . . . . . . . . . . . . . 14

2.3.2 Chevrolet Volt . . . . . . . . . . . . . . . . . . . 17

Chapter 3 Differential as a Variable Transmission . . . . . . . . . . . . . . . . 21

3.1 Differential With Two Inputs And One Output . . . . . . . . . . . . . . . . . . 21

3.1.1 Known Differential RPM Relationships . . . . . . . . . . . . . . . 22

3.1.2 Theoretical Equations For A Non-backdrivable Differential . . . . . . . . . 23

3.2 Experimental Setup . . . . . . . . . . . . . . . . . . . 25

$3.2 .1 \quad$ Data Acquisition . . . . . . . . . . . . . . . . . 28

3.3 Test Procedure . . . . . . . . . . . . . . . . . . . . . . . . . . . . . . . 29

3.4 Test Procedure Limitations . . . . . . . . . . . . . . . . . . . . . . . . . . . . 29

Chapter 4 Test Results . . . . . . . . . . . . . . . . . . . . . . 31

4.1 Electric Motor Characterization . . . . . . . . . . . . . . . . . . . 31

4.2 RPM Verification . . . . . . . . . . . . . . . . . . . . . . . 31

4.3 Load Test - Inputs In The Same Direction $\ldots \ldots \ldots 33$

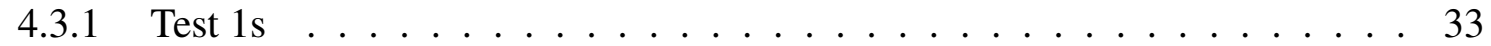

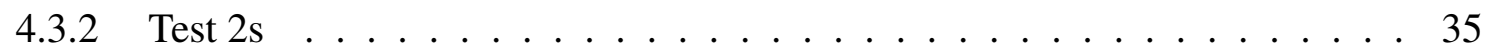

4.4 Load Test - Inputs In Opposite Directions ～. . . . . . . . . . . . . . . . 37

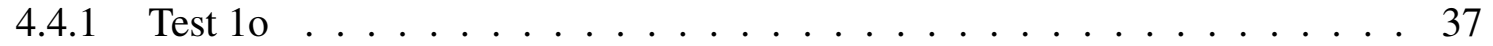

4.4 .2 Test $20 \ldots \ldots \ldots \ldots \ldots$

4.5 Load Test-Cross Over . . . . . . . . . . . . . . . . . . . . . . 41 
Chapter 5 Discussion of Results $\ldots \ldots \ldots \ldots \ldots \ldots$

5.1 RPM Verification . . . . . . . . . . . . . . . . . . . . 45

5.2 Load Test - Inputs In The Same Direction . . . . . . . . . . . . . . . . 46

5.2 .1 Load Test $1 \mathrm{~s} \ldots \ldots \ldots$. . . . . . . . . . . . . . 46

$5.2 .2 \quad$ Load Test $2 \mathrm{~s} \ldots \ldots \ldots$. . . . . . . . . . . . . . . . . . . . 47

5.3 Load Test - Inputs In Opposite Directions ～. . . . . . . . . . . . . . . . . . . 49

$5.3 .1 \quad$ Load Test $10 \ldots \ldots$. . . . . . . . . . . . . . . . . . . . . . . 49

$5.3 .2 \quad$ Load Test $20 \ldots \ldots \ldots$. . . . . . . . . . . . . . . . . 52

5.4 Load Test - Cross Over . . . . . . . . . . . . . . . . . . . . . . . . . 54

5.5 Minimizing Backdrive Between Inputs . . . . . . . . . . . . . . . . 56

5.5 .1 Incorporate A Worm Drive . . . . . . . . . . . . . . . . . 56

5.5.2 Integrate Spur Gear Reduction . . . . . . . . . . . . . . . . 57

5.6 Equations For Gear Reduction $\ldots \ldots \ldots$. . . . . . . . . . . . . . . 61

5.6 .1 Governing Parameters . . . . . . . . . . . . . . . 63

5.7 Planetary Differential . . . . . . . . . . . . . . . . . . . . . . . . . . . 64

5.8 Planetary Differential Advantages . . . . . . . . . . . . . . . . . 67

5.8.1 Advantages Over A Conventional Differential . . . . . . . . . . . . . 68

5.8.2 Advantages Over A Planetary Gear Set . . . . . . . . . . . . . . . . . 69

5.8.3 Practical Constraints Of A Planetary Differential . . . . . . . . . . . 70

5.8.4 Mechanical Differential Comparison . . . . . . . . . . . . . . . . 71

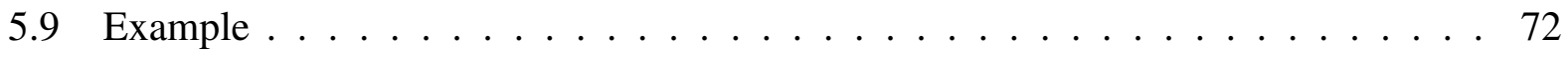

$5.9 .1 \quad$ RPM Implications $\ldots \ldots \ldots \ldots$

5.9 .2 Torque Implications . . . . . . . . . . . . . . . . . . . 76

5.9 .3 Visualization . . . . . . . . . . . . . . . . . . . . . 79

5.10 System Implications . . . . . . . . . . . . . . . . . . . . . . 83

5.10 .1 ICE Implications $\ldots \ldots \ldots$

5.10 .2 Electric Motor Implications ～. . . . . . . . . . . . . . . . . . . . 83

5.10 .3 Peak Torque Requirements . . . . . . . . . . . . . . . . . . . . . . . 84

5.11 Example Summary . . . . . . . . . . . . . . . . . . . . . . . . . . . . . 84

5.12 Results Summary . . . . . . . . . . . . . . . . . . . . 85

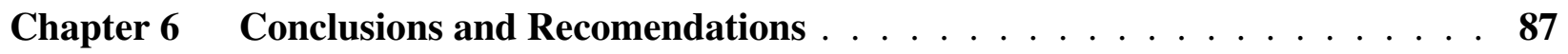

6.1 Restatement Of Research Objectives . . . . . . . . . . . . . . . . . . . . . 87

6.1 .1 Objective $1 \ldots \ldots \ldots \ldots \ldots \ldots \ldots$

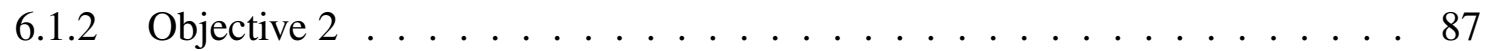

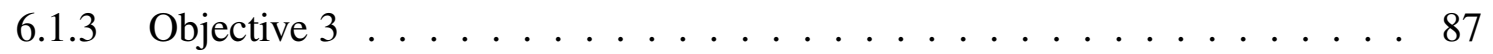

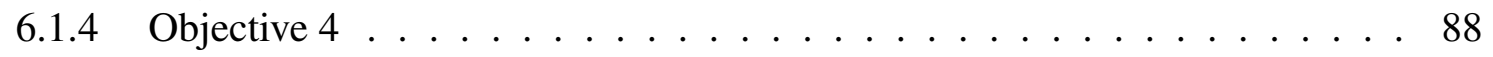

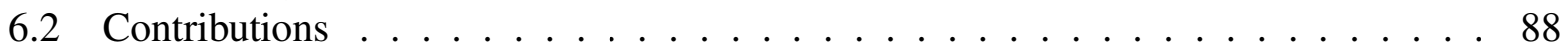

6.3 Additional Contributions . . . . . . . . . . . . . . . . . . . . . . . . 89

6.4 Conclusions . . . . . . . . . . . . . . . . . . . . . . . . . 89

6.5 Recommendations . . . . . . . . . . . . . . . . . . . . . . . 90

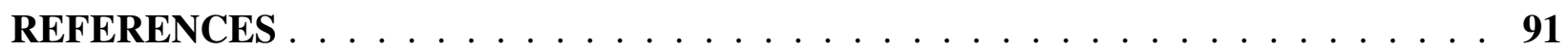

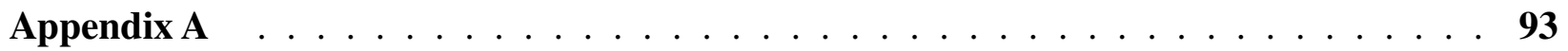


A.1 Experimental Setup . . . . . . . . . . . . . . . . . . . . . . 93

A.1.1 General Layout . . . . . . . . . . . . . . . . . . . . . . 993

A.1.2 Detailed Experimental Wiring Diagram . . . . . . . . . . . . . . . . . 94

A.2 Electric Motor Characterization . . . . . . . . . . . . . . . . . . . . . 95

A.2.1 No Load Speed Test - Varying Field Voltage . . . . . . . . . . . . . . . . 95

A.2.2 No Load Speed Test - Varying Armature Voltage . . . . . . . . . . . . . . 95

A.2.3 Load Test . . . . . . . . . . . . . . . . . . . . . . . 96

A.2.4 Load Test - Field And Armature Trends . . . . . . . . . . . . . . . . 96 


\section{LIST OF TABLES}

2.1 Consumer Economics . . . . . . . . . . . . . . . . . 16

3.1 Differential RPM Relationships . . . . . . . . . . . . . . . . . . . . 22

3.2 Motor Characterization Test - Run 1 . . . . . . . . . . . . . . . 26

3.3 Motor Characterization Test - Run 2 . . . . . . . . . . . . . . . 27

3.4 Motor Characterization Test - Run $3 \ldots \ldots$. . . . . . . . . . . 27

4.1 Test 1s - Motor A and B Armature Voltage Values . . . . . . . . . . . . . . . . . 34

4.2 Test 2s - Motor A and B Armature Voltage Values . . . . . . . . . . . . . 36

4.3 Test 1o - Motor A and B Armature Voltage Values . . . . . . . . . . . . . . . 38

4.4 Test 2o - Motor A and B Armature Voltage Values . . . . . . . . . . . . . . . . 40

4.5 Cross Over Armature Voltage Values . . . . . . . . . . . . . . . . . 42

5.1 Gear Reduction Optimization . . . . . . . . . . . . . . . . . . . 63

5.2 Mechanical Differential Comparison . . . . . . . . . . . . . 71 


\section{LIST OF FIGURES}

1.1 ICE Operating Range . . . . . . . . . . . . . . . . . . . . 3

1.2 Conventional Vehicle Power Flow . . . . . . . . . . . . . . . . 4

1.3 More Ideal ICE Operating Range . . . . . . . . . . . . . . . . . . . . 5

$2.1 \quad$ Vehicle Path . . . . . . . . . . . . . . . . . . . . . . 9

2.2 Differential - 1 Input, 2 Outputs . . . . . . . . . . . . . . . . . 10

2.3 Simplified Differential - Equal Loading _ . . . . . . . . . . . . . . . . . . 11

2.4 Simplified Differential - Unequal Loading . . . . . . . . . . . . . . . . . . . 11

2.5 Common Continuously Variable Transmission . . . . . . . . . . . . . . . . . 12

2.6 Spur Gears . . . . . . . . . . . . . . . . . . . . . . . . . . 13

2.7 Prius Hybrid Powertrain - Power Flow . . . . . . . . . . . . . . . . . 15

2.8 Prius Hybrid Powertrain - Diagram . . . . . . . . . . . . . . . . . . . . . . 16

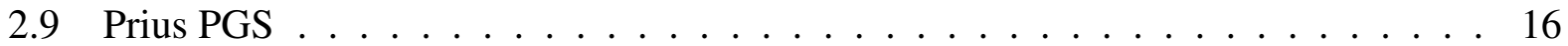

2.10 Series Powertrain . . . . . . . . . . . . . . . . . . . . 17

2.11 Volt PGS . . . . . . . . . . . . . . . . . . . . . 18

2.12 Volt And Prius PGS Comparison . . . . . . . . . . . . . . . . 18

3.1 Differential - 2 Inputs, 1 Output . . . . . . . . . . . . . . . . . 21

3.2 Simplified Differential - 2 Inputs, 1 Output . . . . . . . . . . . . . . . . 21

3.3 Inside A Differential . . . . . . . . . . . . . . . . . . . . 22

3.4 Sign Convention . . . . . . . . . . . . . . . . . . . . . . . . . . 23

3.5 Dual Input Non-backdrivable Differential - Visual . . . . . . . . . . . . . . . . . . 24

3.6 Dual Input Non-backdrivable Differential - Graphical . . . . . . . . . . . . . . 25

3.7 Experimental Setup . . . . . . . . . . . . . . . . . . . . . . 26

3.8 Motor Characterization Results . . . . . . . . . . . . . . . . . . . . . . . . 28

4.1 Experimental Setup - Reference: same as Figure $3.7 \ldots \ldots 32$

4.3 RPM Output Verification - Field Variation . . . . . . . . . . . . . . . 32

4.2 RPM Output Verification - Armature Variation . . . . . . . . . . . . . . . . 33

4.4 Test 1s RPM Output - Same Direction Inputs . . . . . . . . . . . . . . . . . . . . 34

4.5 Test 1s Torque Output - Same Direction Inputs . . . . . . . . . . . . . . . 35

4.6 Test 2s RPM Output - Same Direction Inputs . . . . . . . . . . . . . . . . 36

4.7 Test 2s Torque Output - Same Direction Inputs . . . . . . . . . . . . . . . 37

4.8 Test 1o RPM Output - Opposite Direction Inputs . . . . . . . . . . . . . . . . 38

4.9 Test 1o Torque Output - Opposite Direction Inputs . . . . . . . . . . . . . . . . 39

4.10 Test 2o RPM Output - Opposite Direction Inputs . . . . . . . . . . . . . . . . 40

4.11 Test 2o Torque Output - Opposite Direction Inputs . . . . . . . . . . . . . . 41

4.12 Cross Over RPM Output . . . . . . . . . . . . . . . . . . . . . . . . . 42

4.13 Cross Over Torque Output . . . . . . . . . . . . . . . . . . . . . 43

5.1 RPM Output Verification - Armature Variation: same as Figure 4.2 . . . . . . . . 45

5.2 Test 1s RPM Output - Same Direction Inputs: same as Figure 4.4 . . . . . . . . . . 46

5.3 Test 1s Torque Output - Same Direction Inputs: same as Figure 4.5 . . . . . . . . 47

5.4 Test 2s RPM Output - Same Direction Inputs: same as Figure 4.6 . . . . . . . . . 48 
5.5 Test 2s Torque Output - Same Direction Inputs: same as Figure 4.7 . . . . . . . . . 48

5.6 Non-backdrivable Differential - Visual: same as Figure 3.5 . . . . . . . . . . . . . 49

5.7 Backdrivable Differential - Visual . . . . . . . . . . . . . . . . 49

5.8 Dual Input Non-backdrivable Differential - Graphical: same as Figure 3.6 . . . . 50

5.9 Test 1o RPM Output - Opposite Direction Inputs: same as Figure 4.8 . . . . . . . 51

5.10 Test 1o Torque Output - Opposite Direction Inputs: same as Figure 4.9 . . . . . . 51

5.11 Test 2o RPM Output - Opposite Direction Inputs: same as Figure 4.10 . . . . . . 53

5.12 Test 2o Torque Output - Opposite Direction Inputs: same as Figure 4.11 . . . . . 53

5.13 Test Cross Over RPM Output: same as Figure 4.12 . . . . . . . . . . . . . . . 55

5.14 Test Cross Over Torque Output: same as Figure $4.13 \ldots \ldots 5$

5.15 Minimizing Backdrive - Worm Drive . . . . . . . . . . . . . . . . 57

5.16 Gear Comparison . . . . . . . . . . . . . . . . . . . . . . . . . . . . . . . . . . .

5.17 Gear Efficiency Comparison . . . . . . . . . . . . . . . . 58

5.18 Increasing Torque Flow to Output C . . . . . . . . . . . . . . . . 59

5.19 Backdrivable Differential - Visual: same as Figure 5.7 . . . . . . . . . . . . 59

5.20 Non-backdrivable Differential - Visual: same as Figure 3.5 . . . . . . . . . . . . 60

5.21 Minimizing Backdrive In A Differential - Visual . . . . . . . . . . . . . . . . . 60

5.22 Planetary Differential - Isometric View . . . . . . . . . . . . . . . . . 65

5.23 Planetary Differential - Front View . . . . . . . . . . . . . . . . 65

5.24 Planetary Differential - Side View . . . . . . . . . . . . . . . 66

5.25 Planetary Differential - Top View . . . . . . . . . . . . . . . . . . . 66

5.26 Planetary Differential - Power Transmission . . . . . . . . . . . . . . . 67

5.27 Conventional Differential . . . . . . . . . . . . . . . . . 68

5.28 Planetary Gear Set . . . . . . . . . . . . . . . . . . . . . . . 69

5.29 Planetary Differential - Ratio Optimization . . . . . . . . . . . . . . . . 70

5.30 Mechanical Differential Comparison . . . . . . . . . . . . . 71

5.31 Vehicle Layout Example . . . . . . . . . . . . . . . . . . . 72

5.32 Output RPM Dependencies - Vehicle Stopped . . . . . . . . . . . . . . 73

5.33 Output RPM Dependencies - Low . . . . . . . . . . . . . . . . . . . . . . . . . . . . . . 74

5.34 Output RPM Dependencies - Medium ． . . . . . . . . . . . . . . . . . . 74

5.35 Output RPM Dependencies - High . . . . . . . . . . . . . . . . . 75

5.36 Output RPM Dependencies - Ratio Change . . . . . . . . . . . . . . 76

5.37 Torque Dependencies - Vehicle Accelerating From A Stop . . . . . . . . . . . . 77

5.38 Torque Dependencies - Vehicle Accelerating . . . . . . . . . . . . . . . . . . 77

5.39 Torque Dependencies - Vehicle With High Velocity And Minimal Acceleration . 78

5.40 Torque Dependencies - Vehicle With High Velocity And Minimal Acceleration . 79

5.41 Visualization - Vehicle Accelerating From A Stop . . . . . . . . . . . . . . . . 80

5.42 Visualization - Vehicle Accelerating $1 \ldots \ldots$. . . . . . . . . . . 80

5.43 Visualization - Vehicle Accelerating $2 \ldots \ldots . \ldots . \ldots 81$

5.44 Visualization - Vehicle High Speed . . . . . . . . . . . . . . . . . . 81

5.45 Visualization - Vehicle Reverse . . . . . . . . . . . . . . . . . . 82

5.46 Visualization - Torque and RPM Requirements . . . . . . . . . . . . . . 82

A.1 Setup Diagram . . . . . . . . . . . . . . . . . . 93

A.2 Wiring Schematic . . . . . . . . . . . . . . . . . 94 
A.3 Field Variation $\ldots \ldots \ldots \ldots$

A.4 Armature Variation . . . . . . . . . . . . . . . . . . . . . . 95

A.5 Electic Motor RPM Characterization . . . . . . . . . . . . . . . . . 96

A.6 Electic Motor Torque Characterization . . . . . . . . . . . . . . . . . 96

A.7 Electic Motor Torque Characterization: Trend Line . . . . . . . . . . . . . . . 97 


\section{NOMENCLATURE}

$\mathrm{Ta} \quad$ Input torque from motor $\mathrm{A}$

$\mathrm{Tb} \quad$ Input torque from motor $\mathrm{B}$

Tc Output torque $\mathrm{C}$

rрmA Input rpm from motor A

$r p m B$ Input rpm from motor B

rpmC Output rpm C

$N_{\text {motorA }} \quad$ Number of teeth on Motor A output shaft gear

$N_{\text {inputA }} \quad$ Number of teeth on differential Input A shaft gear

$N_{\text {motorB }} \quad$ Number of teeth on Motor B output shaft gear

$N_{\text {input }} \quad$ Number of teeth on differential Input B shaft gear 


\section{CHAPTER 1. INTRODUCTION}

\subsection{Motivations}

Designing more environmentally friendly and yet affordable vehicles, while maintaining desired performance characteristics, entails many challenges. At the crux of these challenges is the powertrain design, including: power sourcing, controls, systems management, and power transmission. Improvements in any or several of these areas would contribute to the overarching goal of an affordable, environmentally friendly vehicle. Economic and environmental comparisons between conventional, hybrid, electric, and hydrogen fuel cell vehicles reveals that hybrid and electric vehicles have advantages over conventional and hydrogen fuel cell vehicles [1]. Improvements in powertrain design on hybrid and electric vehicles would therefore continue to improve already promising vehicle prospects for the future.

Power transmission from a power source to the traction wheels of a vehicle can be accomplished in various ways. Today's technologies commonly use gear systems integrated with clutches, electric motors, or hydraulics to accomplish the functional specifications required. Other technologies use belts and pulleys in an effort to optimize the power supply or system efficiency. An overview of power transmission systems reveals that the benefits of continuously variable transmissions (CVTs) are substantial in terms of efficiency benefits [2]. However, because many CVT embodiments contain belts and pulleys that rely on friction, there are wear and torque limitations associated with such transmission devices [2]. Torque and wear limitations make these types of CVTs more difficult to integrate into automotive applications.

Recent vehicle technologies have begun to incorporate high voltage power sources, complex monitoring and control systems, and other expensive components. While these efforts are dramatically improving fuel economy, the vehicles have not yet reached a price point that consumers readily accept. In addition to the initial cost of the vehicle, subsequent expensive battery pack replacement is required because of the finite life associated with batteries when compared to 
the life of the vehicle. Future safety and environmental issues associated with such maintenance will be undoubtedly significant.

A transmission that has the ability to use multiple input sources increases the design space for the transmission system to allow a propulsion system design that is more environmentally friendly, while still maintaining desired performance characteristics. For example, a transmission which can obtain power from an internal combustion engine (ICE) and a battery pack can couple the two energy sources to provide the torque necessary to launch a vehicle from rest and allows the ICE to be equipped with less horsepower than otherwise required. Decreasing the ICE horsepower can lead to a decrease in fuel consumption and emissions. Accommodating multiple power transmission inputs also allows the parameters of the battery pack to be optimized, possibly reducing the pack size and thereby the cost of future maintenance and replacement. A multiple-input, singleoutput vehicle transmission is clearly a means of providing a more environmentally friendly and possibly consumer-economic vehicle. Understanding the capabilities of a multiple-input, singleoutput transmission is fundamental to determining what each of the power source inputs must be able to provide.

If hybrid and electric powertrains are to be successful in the future with respect to the environment, consumer economics, performance, and safety, improvements to all subsequent subsystem components and controls are essential. One such subsystem is the transmission, the device used to translate mechanical energy from the power source(s) to the drive wheels.

The prominent challenges associated with current transmissions entail the need for shifting and reliance on friction. The need for shifting is derived from the ICE's limited torque and rpm operating range. An ICE for vehicle applications usually operates between 500 and 4,000 revolutions per minute (rpm), with better torque and efficiency capabilities usually between 1,000-3,000 rpm. Figure 1.1 shows the more efficient operating range for a vehicle ICE.

Along the horizontal axis of Figure 1.1 the engine rpm is referenced. The curves associated with the horizontal rpm values indicate the available torque, power, and fuel consumption at the indicated rpm operating point. An efficient operating point is where high values of available torque and power correlate with low fuel consumption, such as at the 1,800 rpm operating point.

Common road speeds of 60 miles per hour $(\mathrm{mph})$ require the drive wheels to rotate at about $850 \mathrm{rpm}$ (on a 24 inch diameter drive wheel). An engine speed of 1,800 rpm would therefore 


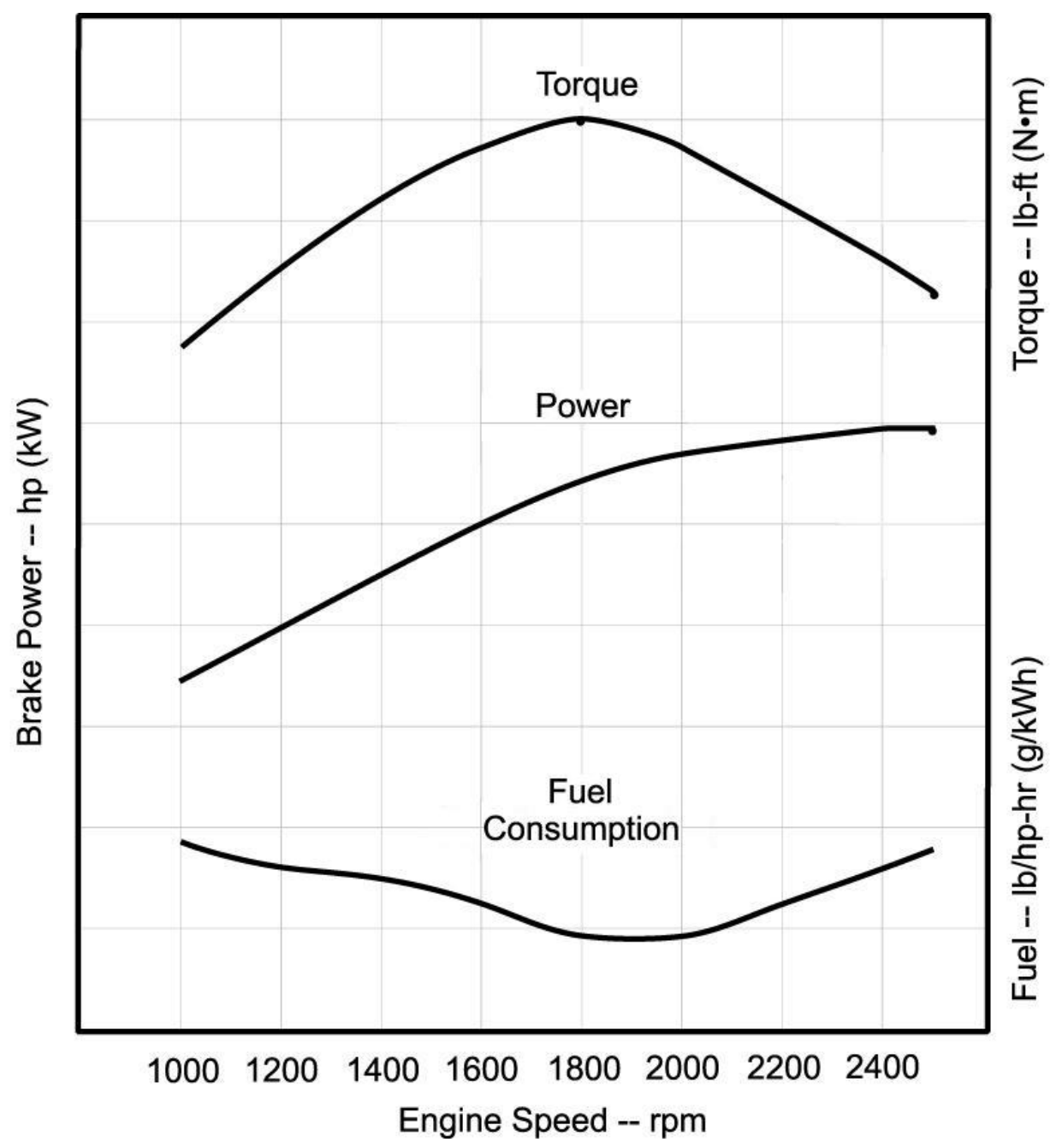

Figure 1.1: ICE Operating Range

require a gear reduction of approximately 2.1:1 gear reduction. Lower traffic speeds of 15-35 mph require drive wheels to operate in the range of 200-500 rpm with a 4:1 reduction. In order for the ICE to operate in a range that enables performance and improved fuel economy, a transmission links the ICE to the drive wheels and provides the necessary gear ratio reductions to better target the best operating range of the ICE at the given driving conditions. Figure 1.2 displays the powerflow from the ICE through the transmission to the drive wheels.

Transmissions typically used in automotive applications are manual or automatic. A manual transmission is a transmission where the driver changes gear ratios via a clutch system at his or her discretion. An automatic transmission requires no driver interaction for shifting during driving and gear ratio changes are governed by the vehicle's electronic engine control unit (ECU). Manual 


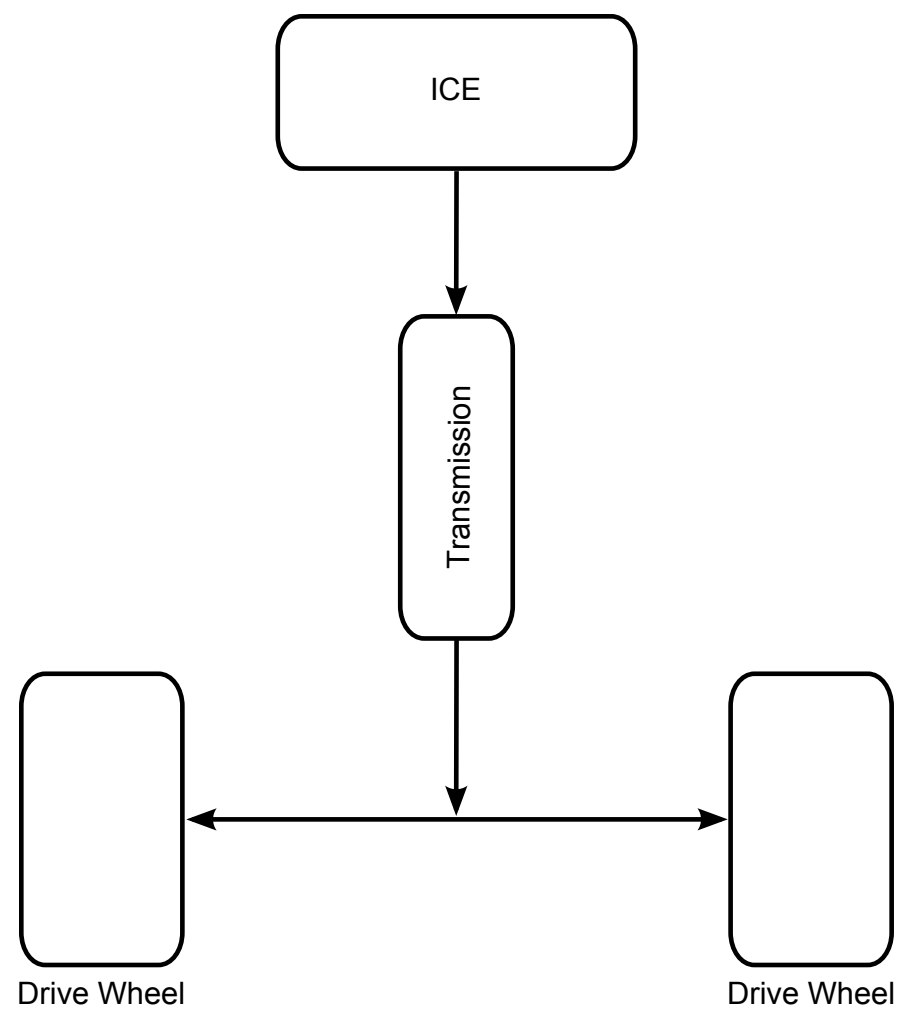

Figure 1.2: Conventional Vehicle Power Flow

and automatic transmissions enable the driving requirements to be met under the practical constraints of the ICE operating range. However, because of the shifting between gears that must take place in manual and automatic transmissions, the engine must still operate in a broader than ideal range. Figure 1.3 exhibits a more efficient operating range for an ICE, one that approaches an ideal operating point. As stated previously, the ideal operating point would be the point at which maximum torque and power are available with minimum fuel consumption. It should be noted that the ideal ICE operating range is dependent on individual engine parameters and would be derived for each specific engine model. Additionally, performance characteristics such as acceleration, hill climbing, and towing call for high torque and make minimal fuel consumption difficult.

The necessity of shifting gear ratios to facilitate overall powertrain performance creates inefficiencies and wasted energy that could otherwise be used for vehicle propulsion. Enabling the ICE to operate at its range of greatest efficiency continuously, instead of regularly varying away from that efficiency range, would improve powertrain efficiency. The elimination of shifting 




Figure 1.3: More Ideal ICE Operating Range

between gear ratios facilitates the operation of an ICE in a more efficient range and thus less fuel consumption is needed for vehicle propulsion.

Recent research which compares simulated efficiencies of a planetary gear hybrid powertrain (PGHP) to that of a conventional continuously variable transmission on the urban route Federal Test Procedure (FTP72) driving schedule revealed that the PGHP did in fact have better efficiency than the conventional CVT [3]. However, physical test data on a PGHP is scarce largely due to the fact that those who have interest in transmission improvements maintain proprietary rights to such data. Torque and rpm relationships in a multiple-input, single-output transmission device are not widely accessible. 
Significant benefits in fuel economy for hybrid vehicle propulsion systems will certainly be obtained if the elimination of shifting between fixed gear ratios can be achieved. For this purpose, the focus of this work will be to investigate the relationships that exist between torque and rpm in a multiple-input, single-output transmission device.

\subsection{Research Objectives}

This research refers often to the term 'non-backdrivable'. The term 'non-backdrivable' will be used in this work to describe a dual-input transmission system, where the input torque on one shaft cannot be transferred to the other input to cause the second input to become regenerative. In essence, if a dual-input, single-output device is 'not backdrivable' both torque inputs are independent of one another.

\subsubsection{Objective 1}

Create a simple graphical model for a dual-input, single-output non-backdrivable differential system.

\subsubsection{Objective 2}

Develop theoretical equations for a non-backdrivable differential gear set that relate multiple input torques and rpms to a single-output torque and rpm.

\subsubsection{Objective 3}

Develop a multiple-input, single-output experiment that can prove or disprove the theoretical equations. Reference the experimental data for the multiple-input sources at the applied loads for the performed tests.

\subsubsection{Objective 4}

Based upon test results, present recommendations for implementation of a differential as a continuously variable transmission (CVT) or infinitely variable transmission (IVT) in a hybrid 
vehicle powertrain. Recommendations will be described in the form of cause-and-effect relationships.

This thesis is developed as follows. Chapter 1 has outlined the inefficiencies associated with shifting between set gear ratios to achieve desired vehicle performance under the practical constraints of an ICE. Also, the first chapter has indicated there are significant advantages for hybrid vehicle propulsion systems that may be found through understanding torque and rpm relationships in a multiple-input, single-output transmission device.

Chapter 2 outlines the function of a differential as most commonly used in automotive applications to date. This overview is followed by a review of existing literature on state of the art hybrid powertrains currently in production.

Chapter 3 delineates the nature of the experimental setup. For experimental purposes a differential was used as the multiple-input, single-output device instead of a planetary gear set, because of the availability of differential gear sets. The similarities between a planetary gear set and a differential gear set regarding multiple-inputs and outputs facilitate the exchange of such apparatus in the experiment. The experimental setup includes a description of how a differential might be used in a powertrain application, known rpm relationships for a differential, theoretical equations for a non-backdrivable differential, and the physical experimental layout.

Chapter 4 includes experimental results and observations.

Chapter 5 discusses the experimental results and states inferences from those results.

Chapter 6 provides conclusions and recommendations for the implementation of a differential as a continuously variable transmission for powertrain applications and suggests future research. 


\section{CHAPTER 2. BACKGROUND}

\subsection{Function Of A Mechanical Differential}

Current vehicles use a differential primarily to allow drive wheels on the same axle to rotate at different rates (rpm). Differentials are designed to allow wheels to turn at different rates to avoid dragging one tire (the tire that travels the furthest circumferential distance) and/or spinning the other tire (the tire that travels the shorter circumferential distance). Figure 2.1 is a graphical representation of the circumferential dissimilar tire paths.

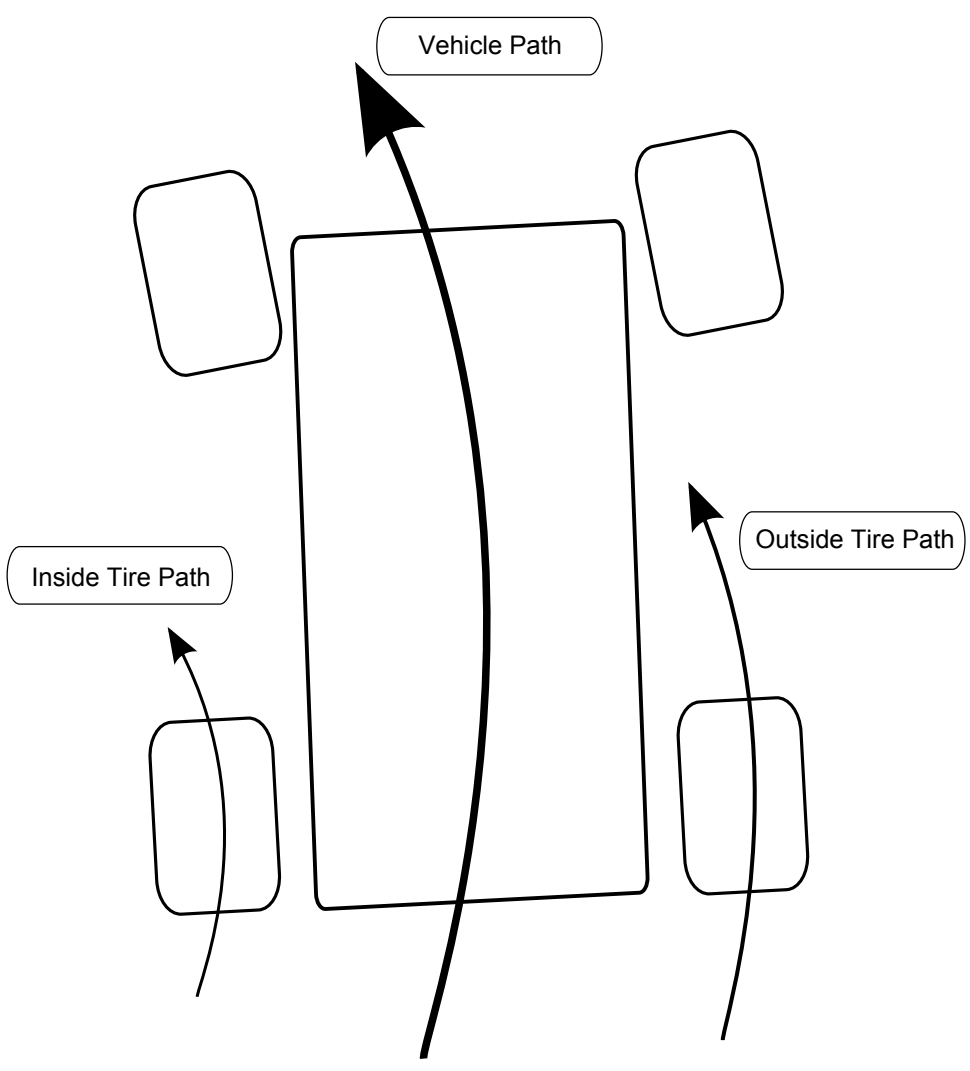

Figure 2.1: Vehicle Path 
Circumferential tire path dissimilarities are especially evident on such driving surfaces as asphalt and concrete, where without a differential to allow the drive wheels to turn at different rates, skid marks would occur as a result of a spinning or dragging tire. In many cases where differentials are used, differing rotational speeds can be accomplished while each of the drive wheels maintains equal torque [4]. Figure 2.2 shows a differential with one rotational input and two rotational outputs, as used commonly today.

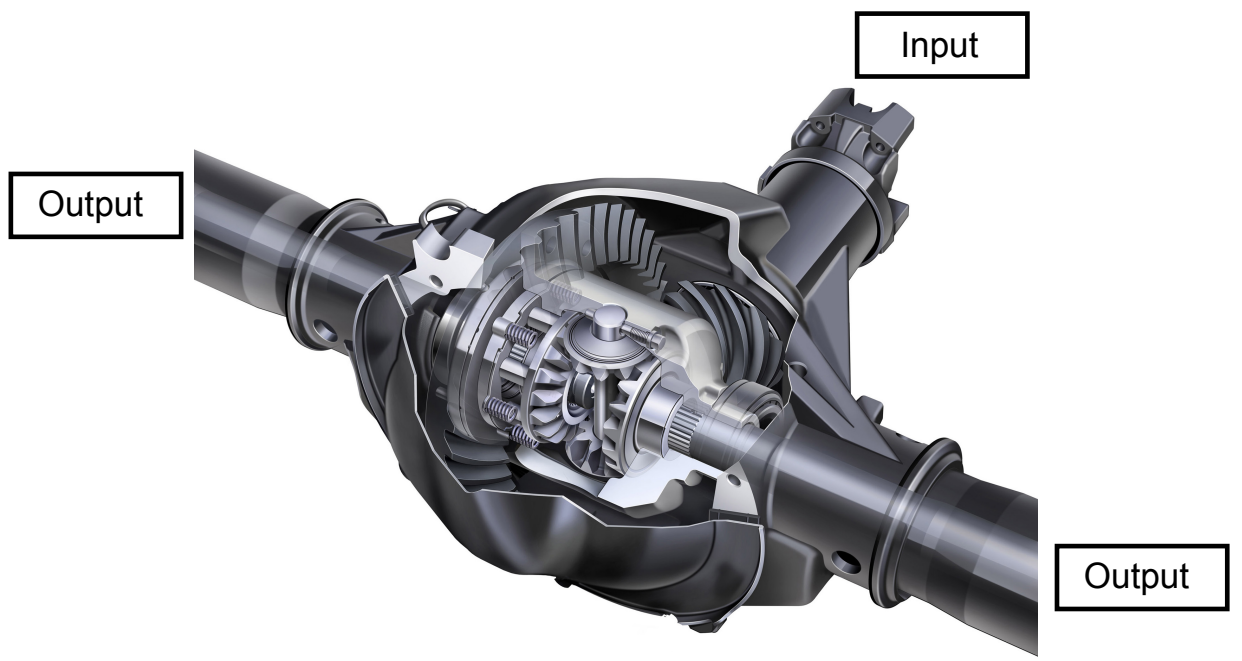

Figure 2.2: Differential - 1 Input, 2 Outputs [5]

Figure 2.3 gives a simplified view of the internal workings of a differential. As can be seen in this figure the large blue ring gear receives an input torque [6]. The input comes from the transmission drive shaft (not shown). The ring gear is fixed to the adjacent blue box carrier and provides torque to both side shafts via the red and yellow bevel gears (which provide power to the wheels). When the resistance load on the wheels is equally distributed on the green bevel gear, it does not rotate and the wheels turn at the same rate [6].

However, when the load to either wheel is increased independently (such as in turning a corner, see Figure 2.1) the green bevel gear begins to rotate and allows the two output shafts to rotate at different rates. Figure 2.4 shows graphically that when one output is stopped (the one on the left) the other output speeds up (the one on the right). A differential that allows the output 


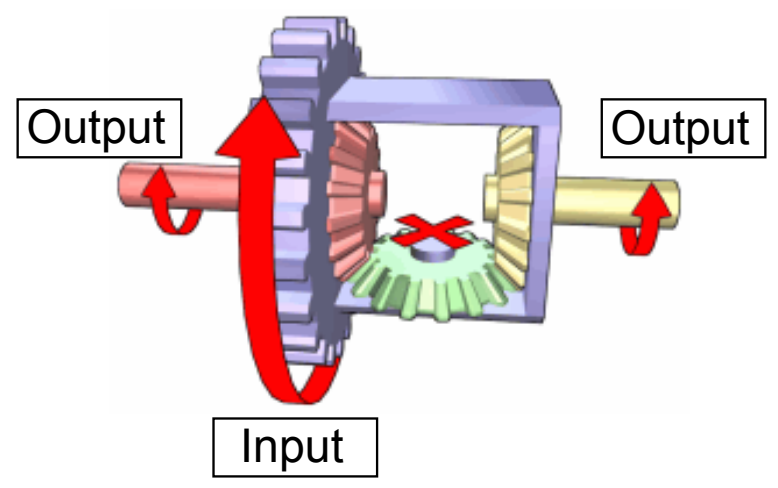

Figure 2.3: Simplified Differential - Equal Loading [6]

shafts to spin at different rates when there is one input infers that there may be potential advantages in changing the two outputs to two inputs and the traditional differential input to an output. The advantage may very well be that the traditional differential could become a transmission that would facilitate multiple inputs. This will be discussed further in Chapter 3.

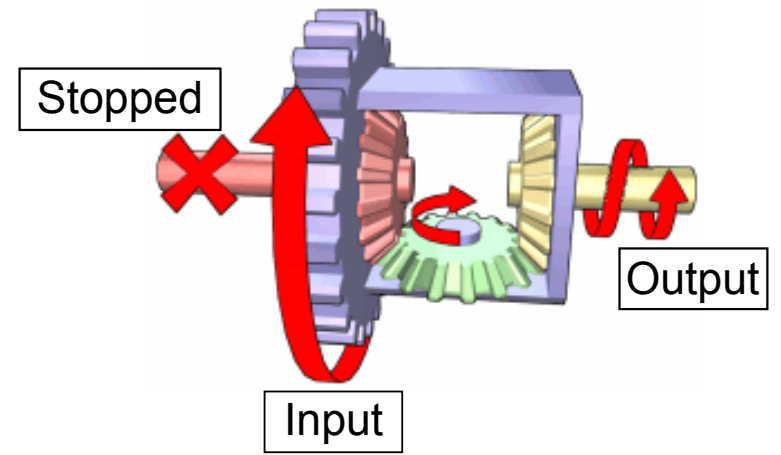

Figure 2.4: Simplified Differential - Unequal Loading [6]

\subsection{Variable Transmissions}

Variable transmissions have significant efficiency advantages over conventional gear shift transmissions in low torque applications $[2,7]$. The basic function of a variable transmission is to change 'gears' continuously to eliminate the need for shifting between gears. By eliminating the need to shift between gears, variable transmissions allow the power source to operate continuously to facilitate better overall efficiency. However, as explicitly outlined by Andersen, current variable 
transmissions which are belt reliant and therefore friction based, have severe disadvantages to conventional gearing in high torque applications. Vehicle propulsion is an application that requires high initial torque during acceleration and makes the direct use of belt type variable transmissions difficult. Figure 2.5 demonstrates a typical belt-driven, continuously variable transmission (CVT).
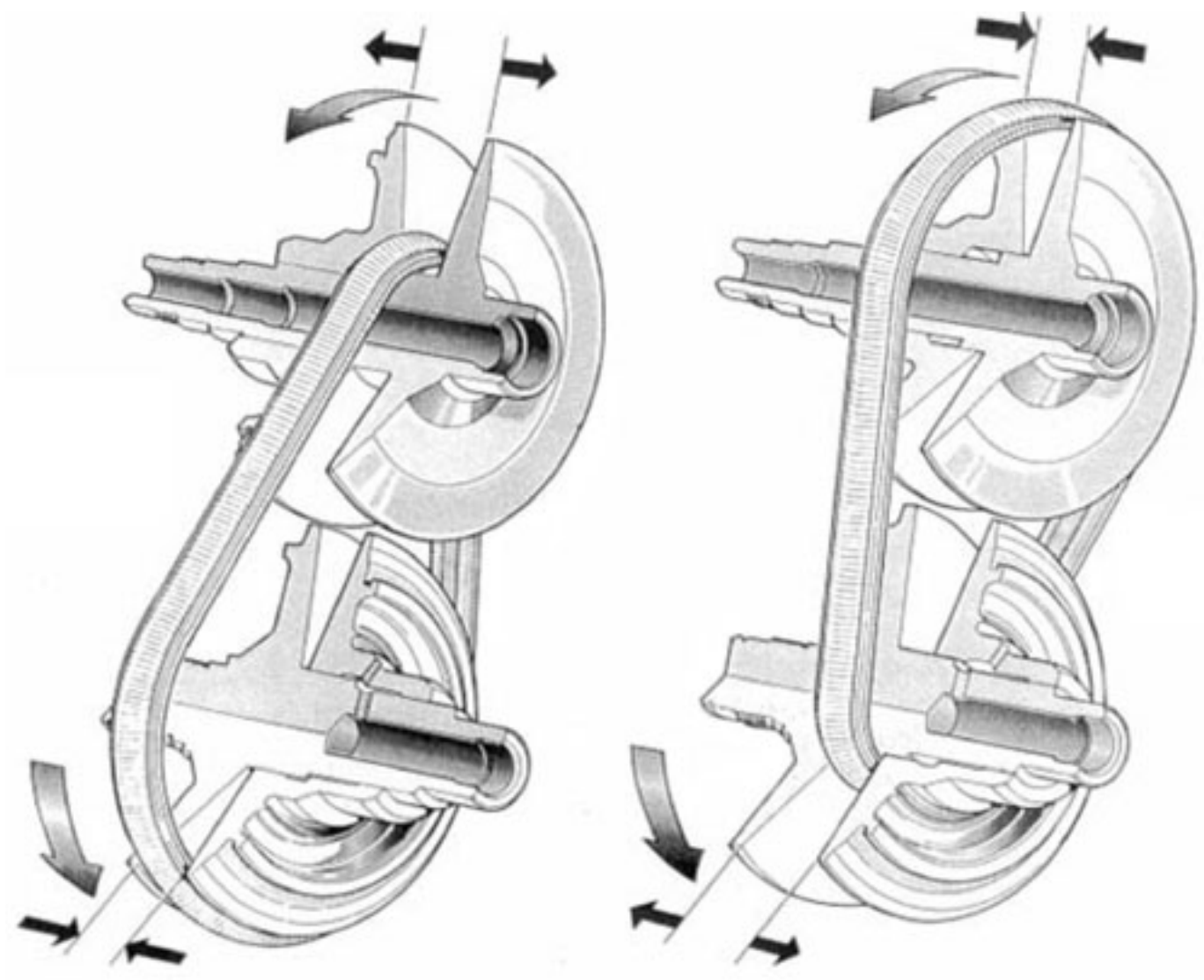

Figure 2.5: Common Continuously Variable Transmission [8]

The fundamental distinction between a continuously and infinitely variable transmission (CVT and IVT respectively) is that a CVT has the ability to change the input to output ratio continuously within a finite range without achieving a zero rotational output. An IVT has the capability to obtain a zero rotational output for nonzero rotational inputs as well as have infinite ratio change [2].

Figure 2.5 shows how a conventional friction dependent CVT works. Initially, at low rpm values, the pulley sheaves resemble the configuration on the left hand side of the figure, with the input pulley at the top and the output pulley at the bottom. Here the top pulley sheaves are spread 
apart creating a small effective diameter and the bottom pulley sheaves are close together making the effective diameter large. The starting configuration functions as a ratio reduction to enable higher torque during acceleration. As the input rpm increases to the top pulley the top pulley sheaves approach one another, increasing the effective diameter of the pulley. When the effective diameter of the top pulley begins to increase, the bottom pulley is loaded by the rotating belt and the sheaves on it begin to separate, producing a gradually smaller effective diameter. Because of this continuous change from one ratio to another ratio with no shifting, the power input is able to operate in a more narrow range. As discussed earlier, allowing the power input to operate in a more narrow range makes higher efficiencies possible, as in the case of the ICE.

The conventional belt driven CVT relies on friction for power transfer from one pulley to another. For low torque applications this is acceptable. For higher torque applications, as in larger vehicle propulsion, friction alone is not reliable because belt slip often occurs [2]. Generally, high torque applications use positively engaged gears, such as those shown in Figure 2.6, to avoid slip. However, using such gears produces a fixed ratio that cannot be varied.

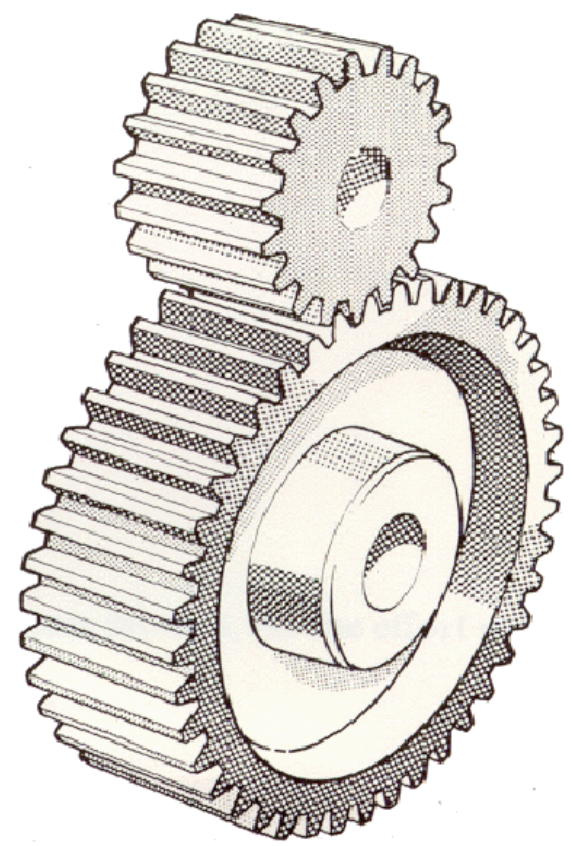

Figure 2.6: Spur Gears [9] 


\subsection{Hybrid Powertrains}

\subsubsection{Toyota Prius}

Recent developments in hybrid powertrains have provided significant fuel economy improvement $[1,3,10]$. Many of these powetrain developments have incorporated the use of planetary gear sets (PGS) in an effort to create a beltless CVT power transmission system. Inventors of such powetrain and subsequent systems go to great lengths to protect such intellectual property [11-16]. For the purpose of protecting such intellectual property much of what is known through testing is not easily accessible to the public. In the case of patents, the disclosure to the public is broad and does not usually contain test data. Writers of patents intentionally make patent applications as broad as possible in an effort to acquire as many rights as possible under that document. The lack of detail in various patents is a strategic move in order to cover as much intellectual property space as possible. Some of what is known about the hybrid powertrains revolves primarily around structural arrangement of components and energy flow. Figure 2.7 displays an energy flow for the Toyota Prius, a leader in hybrid powertrain development.

The Toyota Prius powertrain is based around a parallel or power split transmission device. Figure 2.8 deliniates Figure 2.7 visually, with the power split device in the center of the figure. This power split device is called a planetary gear set (PGS). It uses an electric motor (MG2) and an ICE (here labeled 'Engine') as power inputs and a generating motor (MG1) to control the output ring gear via the PGS (here labeled 'PG') [3]. It is called a power split powertrain because the majority of the power from the ICE goes directly to the wheels via the PGS, while the remainder can be directed to the generator for charging a battery pack. Figure 2.9 shows how the ICE, electric motor, and electric generator are integrated into the PGS.

Controlling the speed of the generator moves the ICE to its optimal operating point via the planetary gear set. The control of the system by the generator (MG1 in Figure 2.9) is made possible by the interconnection of the power/generator sources through the PGS. By using the generator to adjust the system output, this transmission device functions as a CVT [3]. This system configuration also allows the electric motors to function as either power suppliers to the system or energy recovery units, depending on the driving conditions. 


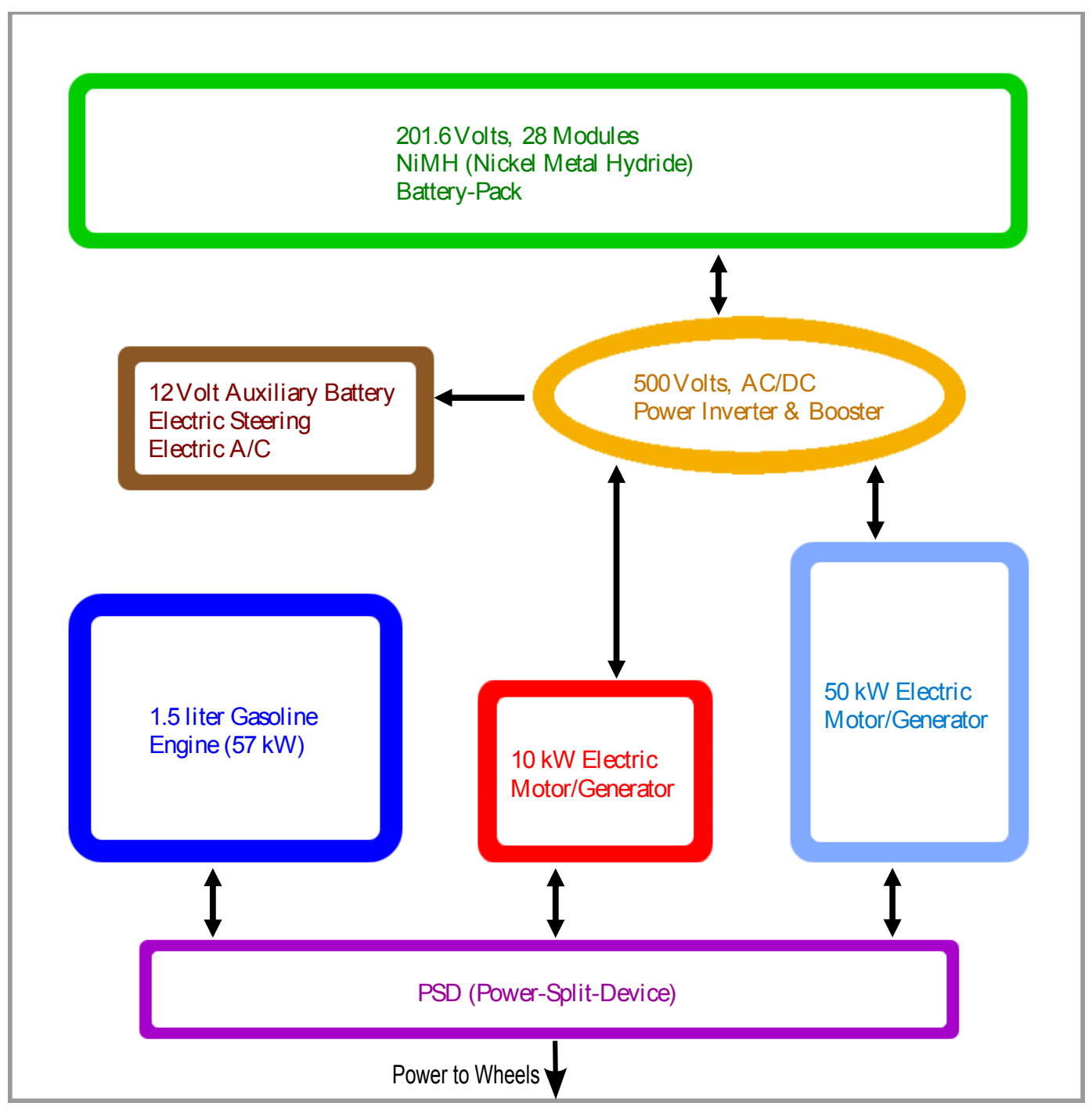

Figure 2.7: Power Flow For Prius Powertrain [17]

Advantages to the Prius powertrain design include outstanding fuel economy and reduced exhaust emissions. In fact, the Prius was certified as a super ultra low emission vehicle (SULEV) and achieved 55 miles per gallon (mpg) at the US EPA highway/city driving cycle [19]. However, the monitoring and control system for this powertrain is severely complex and expensive. In addition, the NHW20 model battery pack operates at 500 volts and will inherently present maintenance and replacement cost issues in the future [20]. Regardless of the much improved fuel economy and drastically reduced emissions, the Toyota Prius is still not consumer economic. A new Prius, including government subsidy, can be purchased at $\$ 22-28,000$. Near the time the purchaser is about to break even on fuel savings the expensive replacement of the Prius' battery pack will be 


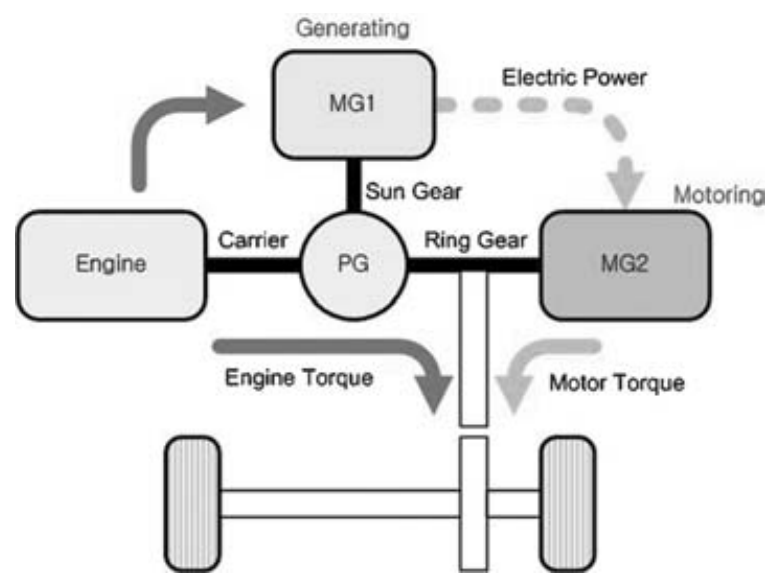

Figure 2.8: Prius Powertrain [3]

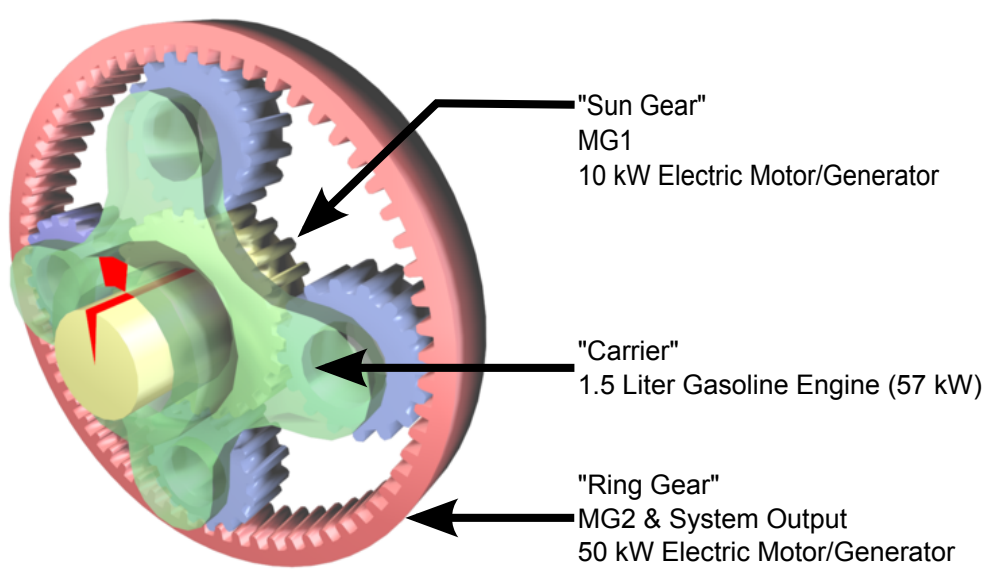

Figure 2.9: Prius PGS [18]

eminent [21]. Table 2.1 outlines consumer cost comparison between a Prius and a conventional four cylinder car for a ten year period, with an average of 15,000 miles per year, and $\$ 3.00$ per gallon.

Table 2.1: 10 Year Consumer Economics: Prius vs. Conventional Vehicle

\begin{tabular}{|c|c|c|c|c|c|c|c|}
\hline $\begin{array}{c}\text { Vehicle } \\
\text { Type }\end{array}$ & MPG & $\begin{array}{c}\text { Initial } \\
\text { Cost }\end{array}$ & $\begin{array}{c}\text { Annual } \\
\text { Fuel Cost }\end{array}$ & $\begin{array}{c}\text { 10 Year } \\
\text { Fuel Cost }\end{array}$ & $\begin{array}{c}\text { 10 Year } \\
\text { Cost }\end{array}$ & $\begin{array}{c}\text { Battery } \\
\text { Replacement }\end{array}$ & $\begin{array}{c}\text { Total } \\
\text { Cost }\end{array}$ \\
\hline Prius & 50 & $\$ 22,000$ & $\$ 900$ & $\$ 9,000$ & $\$ 31,000$ & $\$ 10,000$ & $\$ 41,000$ \\
\hline Conventional & 30 & $\$ 15,000$ & $\$ 1,500$ & $\$ 15,000$ & $\$ 30,000$ & NA & $\$ 30,000$ \\
\hline
\end{tabular}


Table 2.1 clearly outlines that the current Prius does not meet the economical needs of the consumer. For this reason it is essential that improvements be made in hybrid power transmission systems as soon as possible to simplify and enhance consumer appeal. The first step to uncovering the secrets to improvement is to thoroughly understand the torque and rpm relationships of a multiple-input, single-output differential system, which is the primary purpose of this research.

\subsubsection{Chevrolet Volt}

Another hybrid powertrain is the new Chevy Volt series powertrain. Chevrolet refers to the Volt as an extended range electric vehicle because when the battery is charged the Volt is capable of operating on electric power alone. This powertrain uses a plug-in $16 \mathrm{kWh}(8.8 \mathrm{kWh}$ usable) lithium-ion battery pack which supplies electricity to a $111 \mathrm{KW}$ (149 hp) electric motor which is stated as having a range of up to 40 miles $(64 \mathrm{~km})$ on a full charge with fully electric propulsion. In conjunction with the battery pack and the electric motor, a 1.4 liter 4-cylinder ICE runs a 53 KW (71 hp) generator for onboard electricity power generation and propulsion. Having the ICE onboard extends the vehicle travel distance from 40 miles to 300 miles (483 km) [22]. Figure 2.10 shows a power flow schematic of the Chevrolet Volt.

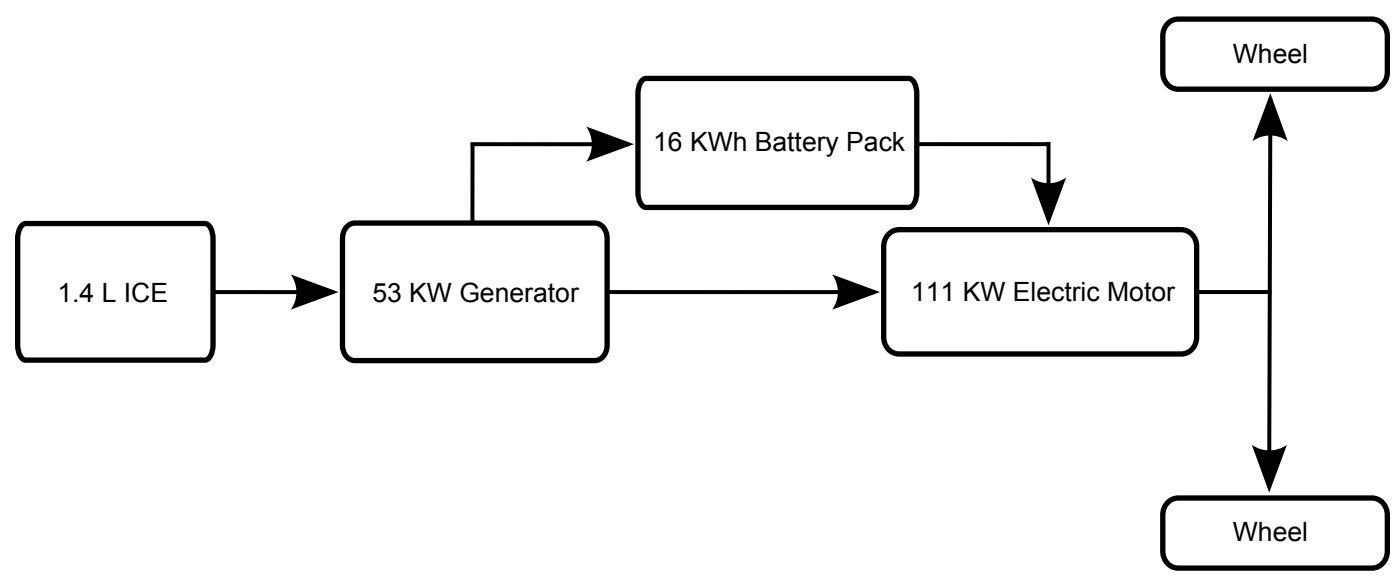

Figure 2.10: Series Powertrain - Volt Powertrain

Although most of the time the ICE does not power the wheels directly, the generator which it does run transfers most of its electric power directly into the electric motor for vehicle propulsion. 
Any excess power is directed to the batteries to maintain a $30 \%$ state of charge on the battery pack [23]. The only time the ICE will power the wheels directly is when a vehicle speed in excess of $70 \mathrm{mph}(113 \mathrm{~km})$ is achieved and the battery pack is depleted to $30 \%$. In this scenario the ICE will aid the electric motor via a PGS. Figure 2.11 displays how the Volt uses a PGS. Figure 2.12 compares how the Volt and Prius differ in their use of a PGS for vehicle propulsion.

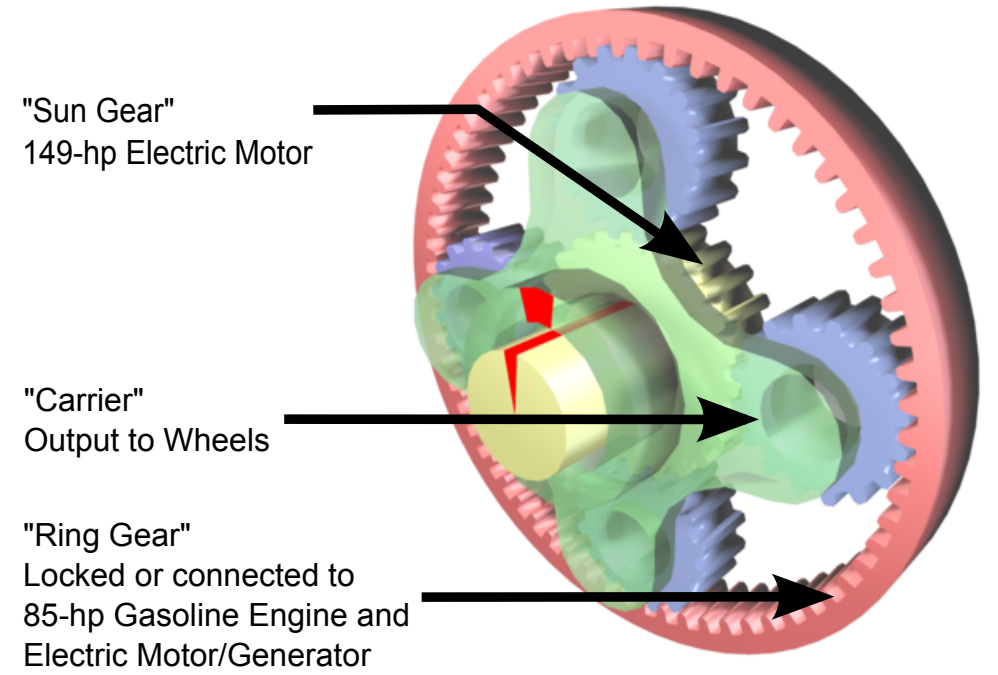

Figure 2.11: Volt PGS [24]

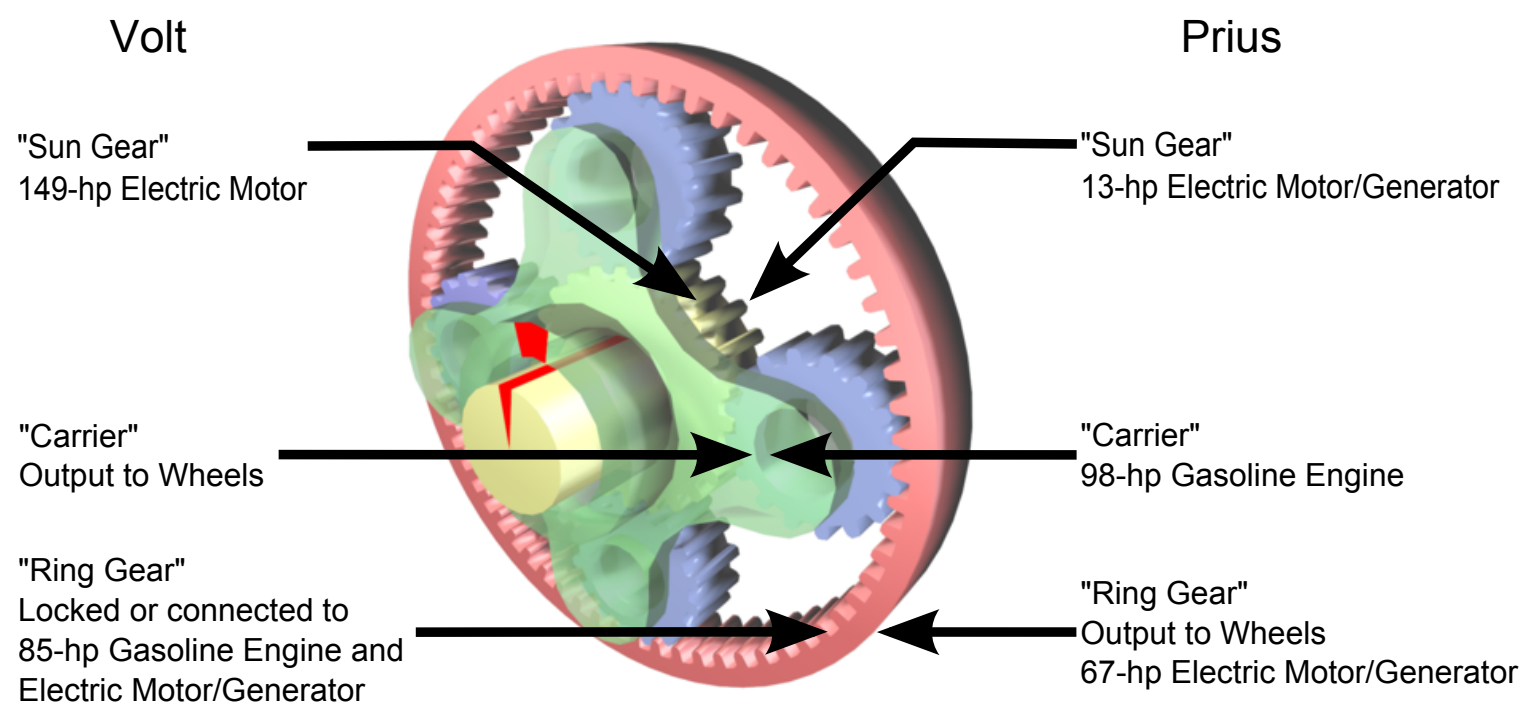

Figure 2.12: Volt And Prius PGS Comparison [24] 
One astute advantage of the Chevrolet Volt series powertrain is that the ICE does not need to run at all in order for the vehicle to be propelled when the battery charge is above $30 \%$. This is unlike the power split design discussed earlier, where the ICE must be running for the vehicle to be propelled faster than a speed of $15 \mathrm{mph}$. The Volt powertrain is especially nice for $75 \%$ of Americans who drive less than 33 miles $(53 \mathrm{~km})$ on their daily commute [22]. The potential reduction in emissions if consumers regularly fully charged their Chevrolet Volt at home would indeed have a large positive environmental impact.

Unfortunately, the projected November 2010 release price is projected to be $\$ 33,500$ after a $\$ 7,500$ government tax credit, making the list price $\$ 41,000$. This is more than a Mercedes Benz C300 Sport Sedan. Once again, it is not realistic that major portions of the consumer market will spend the capital required for initial high costs and impending expensive battery pack replacement. While the battery pack is certainly one of the major cost drivers in the Volt powertrain design, there are other drivers that add substantial portions to the overall cost. Larger than normal electric drive motors are needed in the series drivetrain design, because there is no power transmission device other than a reduction gearbox. Also, the speed of the electric motor is controlled by an expensive solid state controller. These large electric motors in conjunction with the large battery pack necessitate complicated control systems and again increase costs associated with the powertrain and ultimately the vehicle. Another important note to this series powertrain is that once the battery pack falls to a $30 \%$ state of charge the ICE will start and the generator powered by the ICE will only maintain the $30 \%$ state of charge in the battery pack, as the majority of the power is directed from the ICE through the generator to the drive wheels. Maintaining the $30 \%$ state of charge extends battery life, but decreases the extent of the driving range significantly. In comparison to a normal 4-cylinder vehicle with a conventional automatic transmission, the Chevrolet Volt - on a full charge and a full tank, will get about 100 miles less total travel distance. It should be noted however that the Chevy Volt's fuel tank capacity is less than 10 gallons (37.9 liters), while a traditional 4-cylinder vehicle typically has a fuel tank capacity of about 15 gallons (56.8 liters). If only gasoline fuel consumption is considered, not electricity, the Chevy Volt's miles per gallon of gasoline fuel consumption will be about the same as a conventional 4-cylinder gasoline vehicle.

Current hybrid vehicle powertrain technologies, although sophisticated, will not have a large positive impact on environmental issues until they are readily used by the public. The public 
will not freely endorse hybrid vehicles as long as purchase prices on these vehicles are higher than other traditional vehicles. If there is to be any improvement concerning environmental issues regarding personal vehicle transportation it must be economically persuasive to the public eye. For this reason it is essential that the fundamental parameters of torque and rpm relationships in a dual-input, single-output differential system be accessible to the public to encourage further development of a low-cost powertrain design. 


\section{CHAPTER 3. DIFFERENTIAL AS A VARIABLE TRANSMISSION}

\subsection{Differential With Two Inputs And One Output}

A differential can be used as a variable transmission. As described previously, a differential is normally used as shown in Figures 2.2, 2.3, and 2.4, with a single input and two outputs (one output to each wheel). However, when used in the manner shown in Figure 3.1, a differential can accommodate two inputs and a single output. Figure 3.2 can also be referred to for clarification concerning the internal workings of a differential when it is used in the same way as in Figure 3.1.

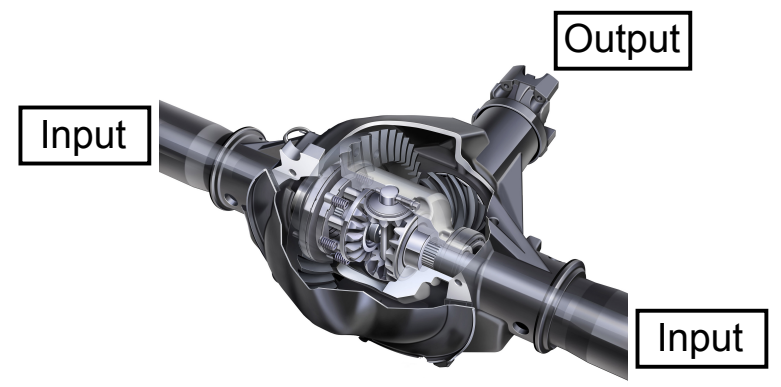

Figure 3.1: Differential - 2 Inputs, 1 Output [5]

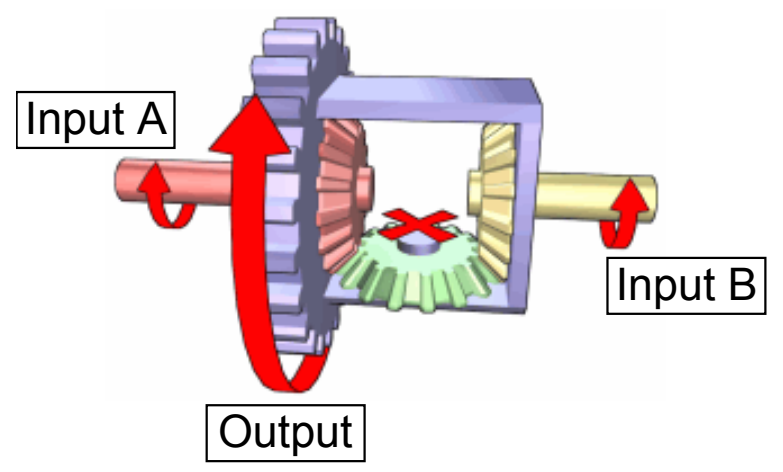

Figure 3.2: Simplified Differential - 2 Inputs, 1 Output [6] 
Because a differential is composed of gears, the input(s) and output(s) remain mechanically connected. Figure 3.3 displays a cutaway of a differential and shows the mechanical connections entailed.

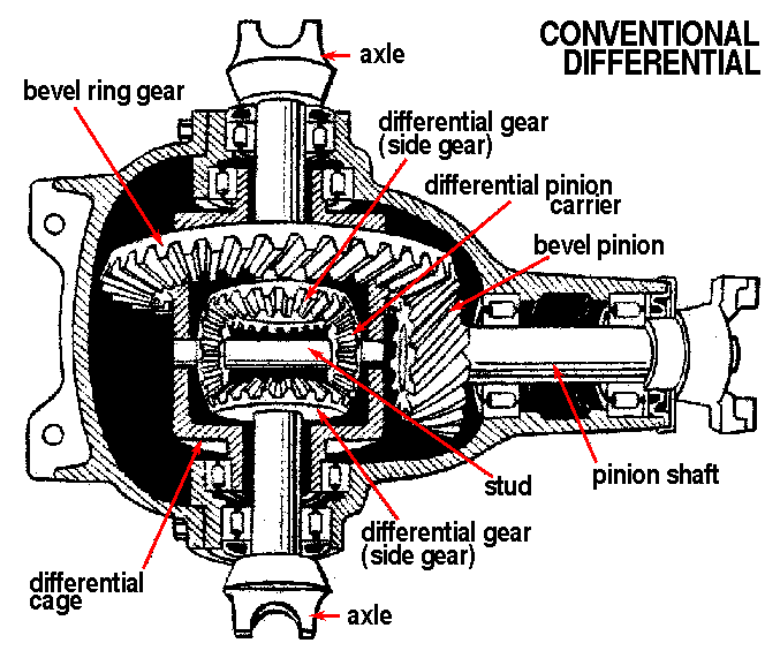

Figure 3.3: Differential Cutaway [25]

\subsubsection{Known Differential RPM Relationships}

Understanding that a differential is mechanically geared, rpm equations can be developed [4]. Table 3.1 presents cause and effect relationships when one differential input is held constant and the other is varied. The inputs and output can be referenced in Figure 3.1 or 3.2 for Table 3.1.

Table 3.1: Differential RPM Relationships

\begin{tabular}{|c|c|c|}
\hline Input A & Input B & Output \\
\hline High & High (Same Direction) & High \\
\hline High & Zero & Medium \\
\hline High & High (Opposite Direction) & Zero \\
\hline
\end{tabular}




\subsubsection{Theoretical Equations For A Non-backdrivable Differential}

The theoretical rpm equation for a dual input single output differential with no gear reduction is as follows:

$$
r p m C=\frac{r p m A+r p m B}{2}
$$

where $\operatorname{rpm} A$ and $\mathrm{rpmB}$ are the input rpm values of the respective shafts and $\mathrm{rpmC}$ is the rpm value of the output shaft or disc. It is important to note the sign convention that will be used throughout the remainder of this work. Positive torque and rpm values will be according to Figure 3.4, as this is the actual Gleason-Torsen differential used in testing.

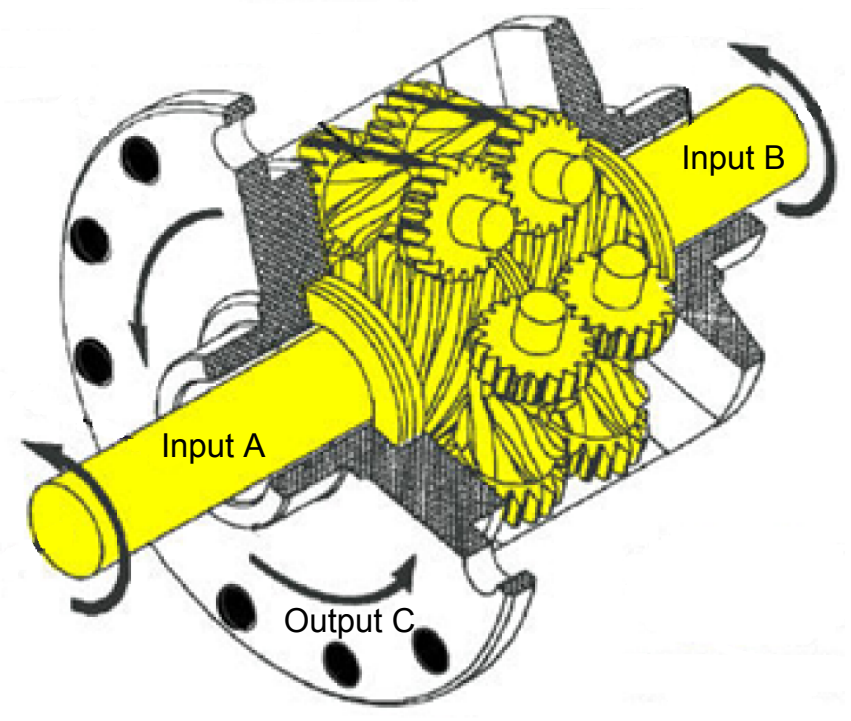

Figure 3.4: Positive Torque and RPM Values

The conservation of power equation may be expressed as the sum of their torques multiplied by the respective angular velocities, below (neglecting frictional losses)

$$
T a \cdot r p m A+T b \cdot r p m B+T c \cdot r p m C=0
$$

where $\mathrm{Ta}, \mathrm{Tb}$, and $\mathrm{Tc}$ are the shaft input and output torques as shown in Figure 3.4. Solving for $\mathrm{Tc}$ we get 


$$
T c=\frac{-T a \cdot r p m A-T b \cdot r p m B}{r p m C}
$$

Substituting equation (3.1) into (3.2) for rpmC, the theoretical non-backdrivable differential governing equation is obtained as:

$$
T c=-\frac{2(T a \cdot r p m A+T b \cdot r p m B)}{r p m A+r p m B}
$$

A theoretical graphical representation of torque flow through a dual-input non-backdrivable differential is helpful in understanding the definition of the term non-backdrivable. Figure 3.5 represents the theoretical torque flow through a dual-input non-backdrivable differential, illustrating visually the theoretical equation (3.4).

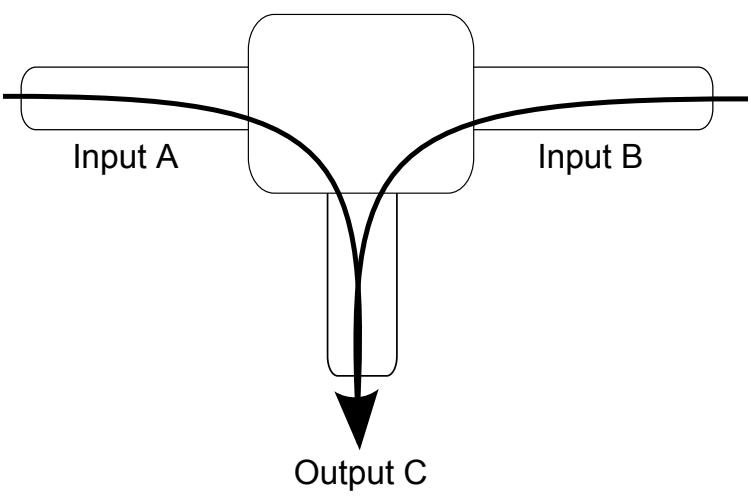

Figure 3.5: Power Flow of a Dual-input Non-backdrivable Differential

The theory behind the torque flow through a differential implies that both of the input shafts are independent of one another with respect to torque, but dependent with respect to rpm. Graphically, equation (3.4) indicates that the output torque, Tc, goes to infinity as the output rpm, rpmC, approaches zero, as in Figure 3.6.

While this is not reasonable in practice, it leads to the question of what the real limitations are to a differential in a power transmission application. In a conventional differential, where there are two outputs, the rpm can differ while the torque to each wheel can remain the same [4]. The torque relationships that occur when there are two inputs and one output is the primary focus of 


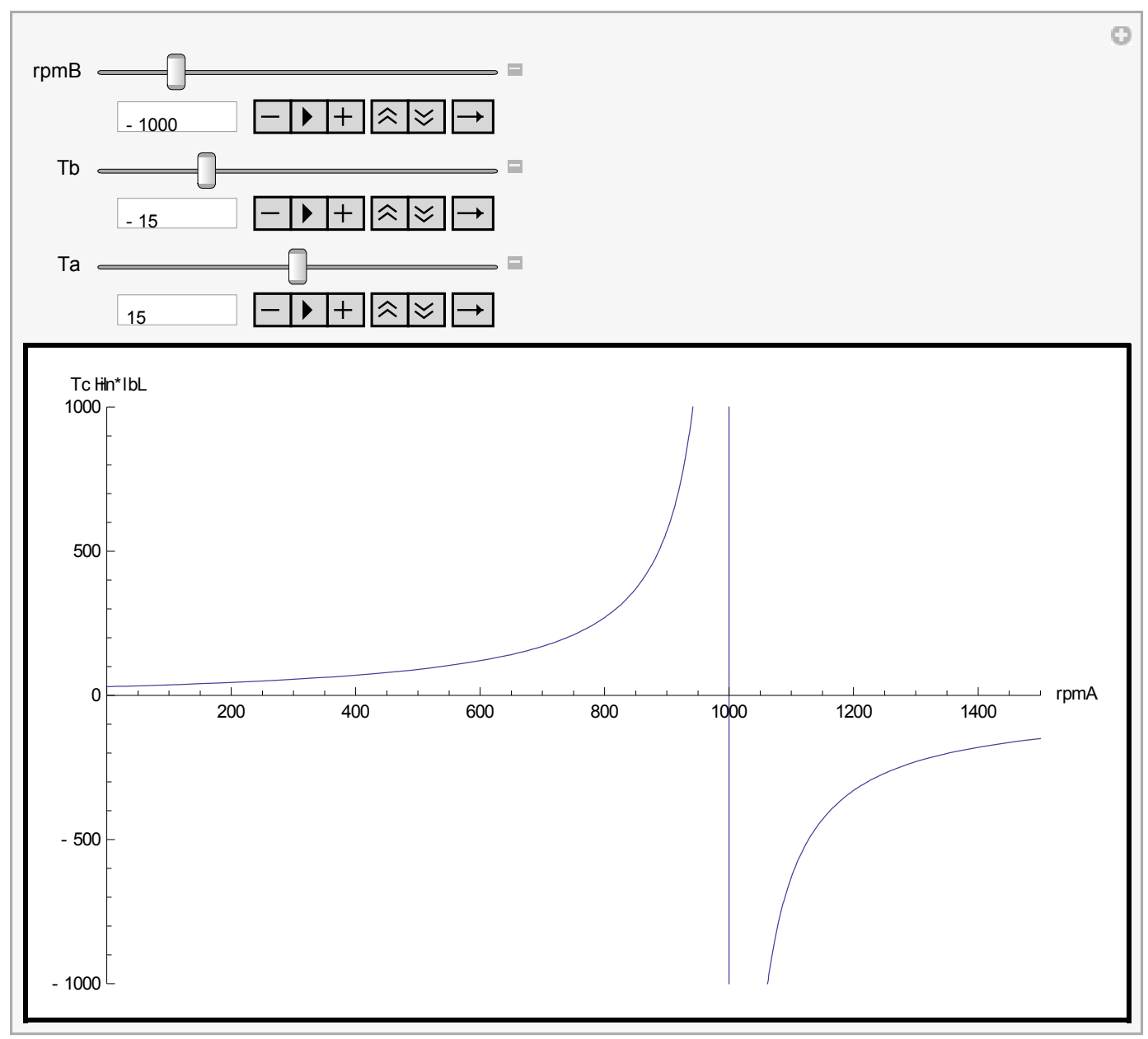

Figure 3.6: Graphical Representation of Equation (3.4)

this work. The results of this research will help determine if there are potential applications for the use of a differential in hybrid or fully electric power transmission systems.

\subsection{Experimental Setup}

To better understand the relationships that occur in a dual-input, single-output differential, an experimental setup has been constructed. This experimental setup includes four power supplies, two electric motors, a differential (as shown in Figure 3.4), and data acquisition equipment. Full experimental and wiring diagrams can be viewed in Appendix A. For visualization of the experimental setup refer to Figure 3.7. 


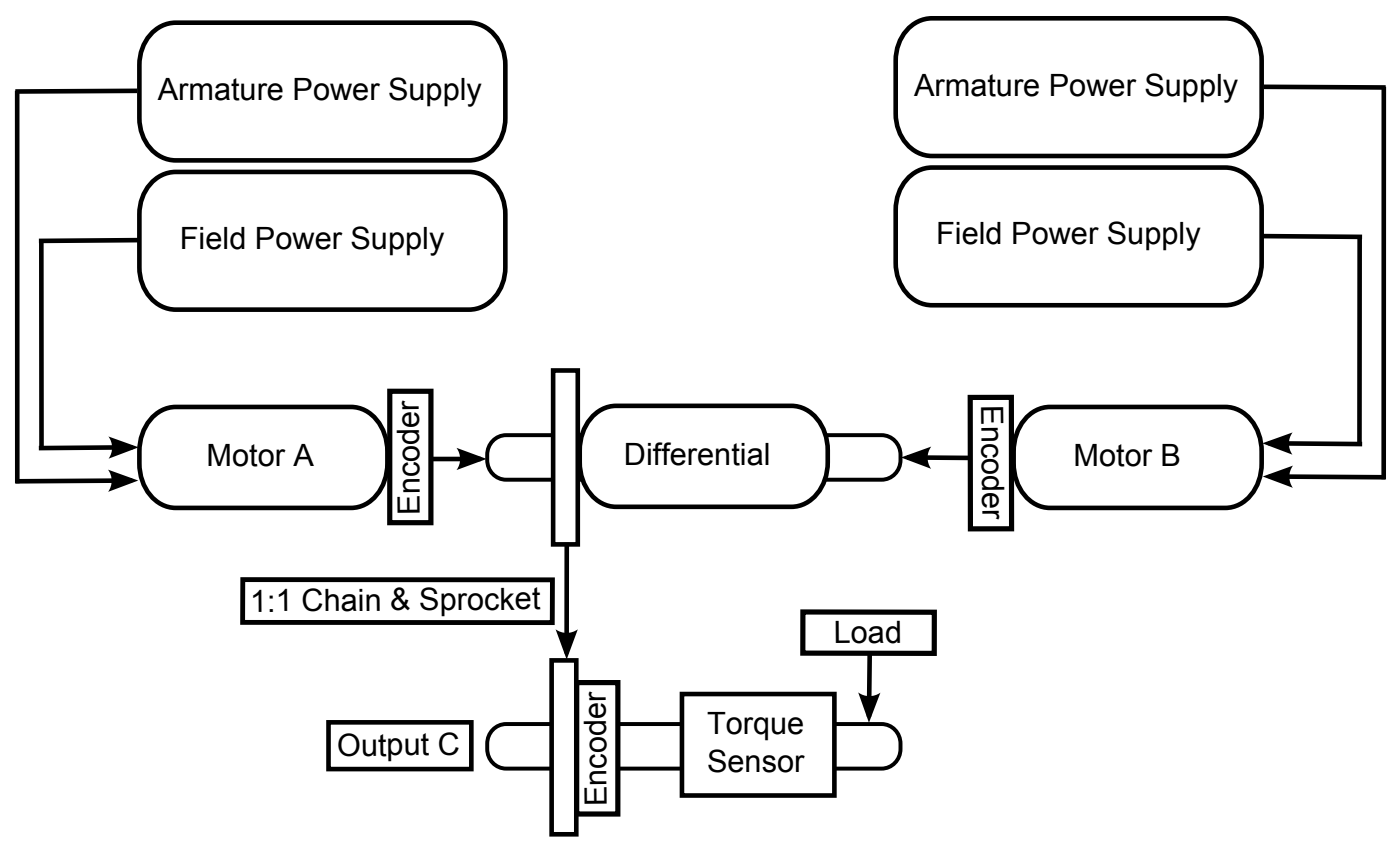

Figure 3.7: Experimental Setup

Before testing with the differential, preliminary motor characterization tests were carried out. These electric motor characterization tests allowed the armature current of each motor to be recorded and translated into input torque for the system. The reason this correlation could be made is that the load on an electric motor shaft determines the amount of current the armature pulls, independent of rpm. Tables 3.2-3.4 indicate the motor characterization test settings and resultant armature and field current values. The upper block in the table specifies the settings for the test and the lower block shows the current drawn for those settings.

Table 3.2: Motor Characterization Test - Run 1

\begin{tabular}{|c|c|c|c|}
\hline & \multicolumn{3}{|c|}{ Test Settings/Results } \\
\hline Motor Field Voltage & 50 & 50 & 50 \\
\hline Motor Armature Voltage & 50 & 50 & 50 \\
\hline Approximate Motor RPM & 1,000 & 1,000 & 1,000 \\
\hline Approximate Load (in $\cdot \mathrm{lbs})$ & 1 & 3 & 4.5 \\
\hline \hline Approximate Armature Current (Amps) & .5 & 1 & 1.5 \\
\hline Approximate Field Current (Amps) & .6 & .6 & .6 \\
\hline
\end{tabular}


Table 3.3: Motor Characterization Test - Run 2

\begin{tabular}{|c|c|c|c|}
\hline & \multicolumn{4}{|c|}{ Test Settings/Results } \\
\hline Motor Field Voltage & 50 & 50 & 50 \\
\hline Motor Armature Voltage & 30 & 30 & 30 \\
\hline Approximate Motor RPM & 600 & 600 & 600 \\
\hline Approximate Load (in $\cdot$ lbs $)$ & 1 & 3 & 4.5 \\
\hline \hline Approximate Armature Current (Amps) & .5 & 1 & 1.5 \\
\hline Approximate Field Current (Amps) & .6 & .6 & .6 \\
\hline
\end{tabular}

Table 3.4: Motor Characterization Test - Run 3

\begin{tabular}{|c|c|c|c|}
\hline & \multicolumn{4}{|c|}{ Test Settings/Results } \\
\hline Motor Field Voltage & 50 & 50 & 50 \\
\hline Motor Armature Voltage & 10 & 10 & 10 \\
\hline Approximate Motor RPM & 150 & 150 & 150 \\
\hline Approximate Load (in $\cdot l b s)$ & 1 & 3 & 4.5 \\
\hline \hline Approximate Armature Current (Amps) & .5 & 1 & 1.5 \\
\hline Approximate Field Current (Amps) & .6 & .6 & .6 \\
\hline
\end{tabular}

As can be seen in the tables, regardless of the motor rpm, the load dictates the amount of current the armature draws. It is also observed that regardless of the load the field current remains generally constant. The correlation between load and armature current facilitates understanding of motor torque input for later testing. Specifically, by recording the armature current during differential testing, a known value for motor torque provided to the system can be derived.

The data is displayed graphically in Figure 3.8. The correlation between torque output for the electric motor and current drawn by the armature is clearly evident. Observe again that although armature voltage values (and thus rpm values) vary between tests, the amount of torque provided due to loading yields extremely similar values of current drawn in the armature. Further characterization tests were done with respect to the armature and field voltage values and are available in Appendix A. For the testing done in the remainder of this work the characterization just described will be the fundamental basis for translating current drawn by the armature in to electric motor torque provided to the system. 


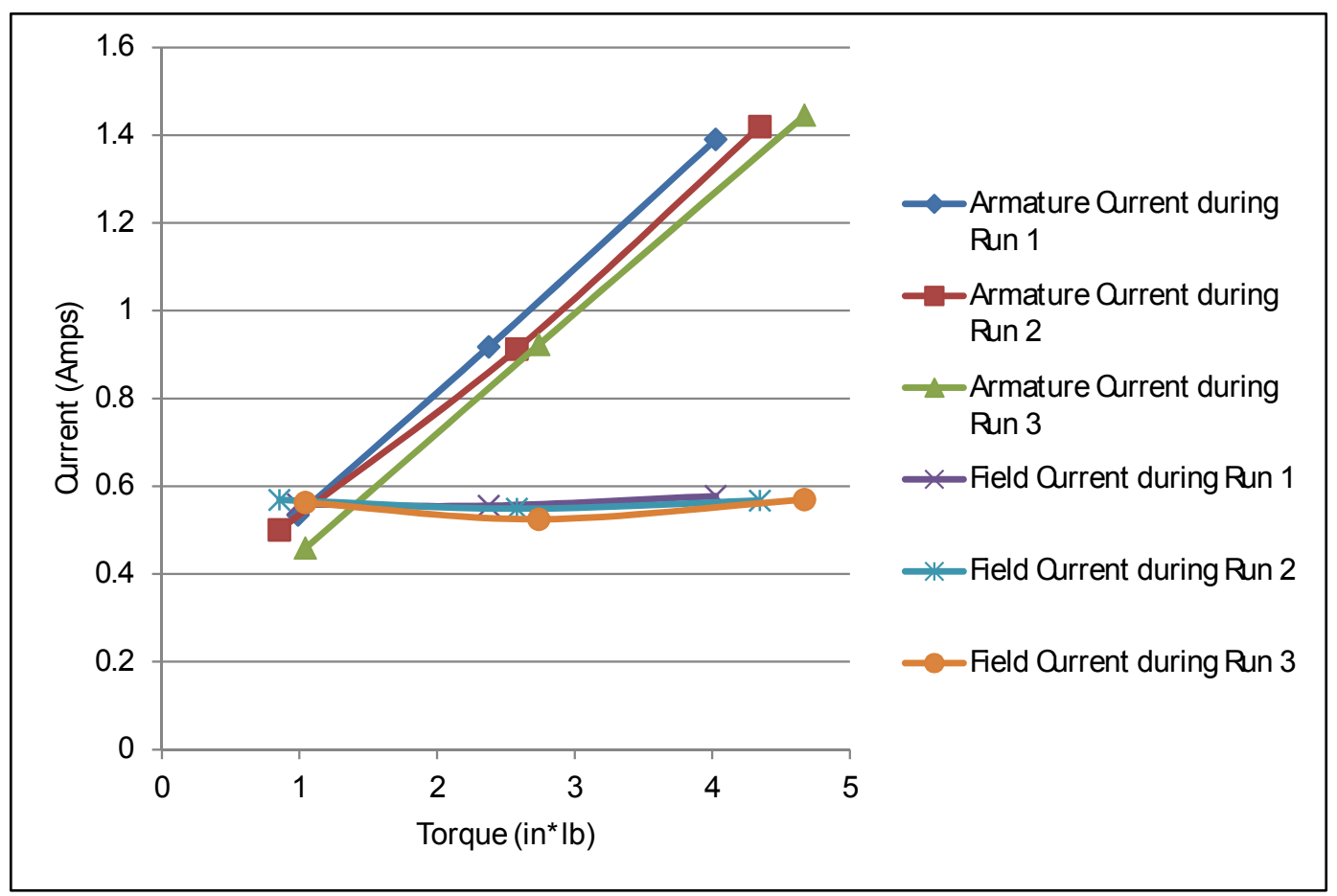

Figure 3.8: Motor Characterization Results

The equation determined for this experiment to translate the amount of current drawn by the armature to the value of torque provided to the system was derived by averaging the three data points at each load location. The equation of correlation used for this experiment was

$$
\text { Motor Torque }=(2.5692)(\text { Armature Current })-0.0701
$$

Equation (3.5) can be viewed graphically in Appendix A.

\subsubsection{Data Acquisition}

The data acquisition system allowed the inputs to, and the outputs from, the system to be monitored and recorded. Instrumentation used in this experiment included a compact real-time input output (cRIO) device, encoders, a torque sensor, and a LabVIEW program. The parameters measured, correlated, and recorded by this instrumentation during this experiment were:

1. The input torque from each motor into the system 
2. The input rpm from each motor into the system

3. The output torque of the system

4. The output rpm of the system

Monitoring these parameters facilitated understanding of overall system power input and output. Also, specific data sets could be tracked to better identify trends.

\subsection{Test Procedure}

The general test procedure used on this experimental setup consisted of the following:

1. An initial voltage setting on the armature and field of each motor

2. The acquisition of rpm, voltage, current and torque data at the initial setting

3. Changing of the armature and/or field voltage settings to one input, 'Motor B'

4. Acquiring data while loading the differential (see Figure 3.7)

5. Repeating steps 3 and 4 until armature and/or field settings on the Motor(s) could no longer be adjusted because of power supply or motor design constraints

Testing in this way allowed voltage, current, rpm, and torque data to be acquired for a range of settings.

\subsection{Test Procedure Limitations}

Physical design of the electric motors constrained this experiment to the following capabilities:

1. Maximum input torque from each motor of $10 \mathrm{in} \cdot \mathrm{lbs}$

2. Maximum output from each motor of $1,700 \mathrm{rpm}$ 


\section{CHAPTER 4. TEST RESULTS}

\subsection{Electric Motor Characterization}

Two separately-excited shunt wound motors were used for the inputs to a differential. These motors were chosen because of their unique capability of being able to 'excite', or supply, the field and the armature independently with voltage and current. The ability to control the electric motors by varying the voltage to the armature, field, or both, has system control implications which will not be discussed in depth in this work. For an in-depth study with regards to electric motors, refer to Benjamin Groen's thesis entitled 'Investigation of DC Motors for Electric and Hybrid Electric Motor Vehicle Applications with an Infinitely Variable Transmission' [26]. However, because this work refers frequently to electric motor constructs, a summary of the characterization tests for the separately excited shunt wound motors is as follows:

1. Voltage applied to the electric motor armature relates linearly to motor output rpm

2. Current drawn by the electric motor armature relates linearly to motor output torque

3. Voltage applied to the electric motor field relates nonlinearly to the motor output rpm

4. Current drawn by the electric motor field correlates negligibly with motor output torque

\subsection{RPM Verification}

The first set of differential test results confirmed Equation (3.1) as indicated in Figures 4.2 and 4.3. Equation (3.1) stated that the output rpm, rpmC, would be the average of the sum of the input rpm values, rpmA and rpmB. For reference, Equation (3.1) and Figure 3.7 are displayed below as Equation (4.1) and Figure 4.1.

$$
r p m C=\frac{r p m A+r p m B}{2}
$$




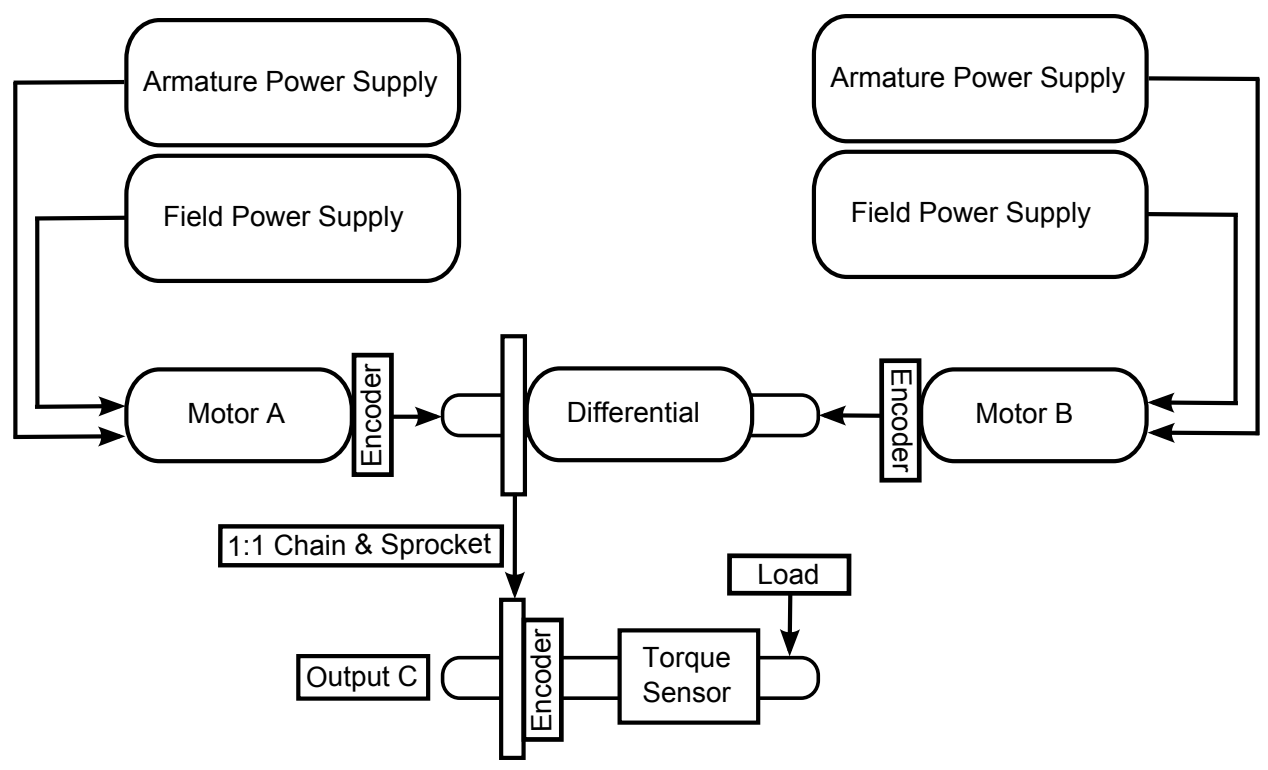

Figure 4.1: Experimental Setup: same as Figure 3.7

Figures 4.2 and 4.3 display the input rpm of each electric motor, the theoretical output rpm, and the actual output rpm (rpmC). It is observed in these figures that the theoretical output of rpmC coincides with the measured output of rpmC.

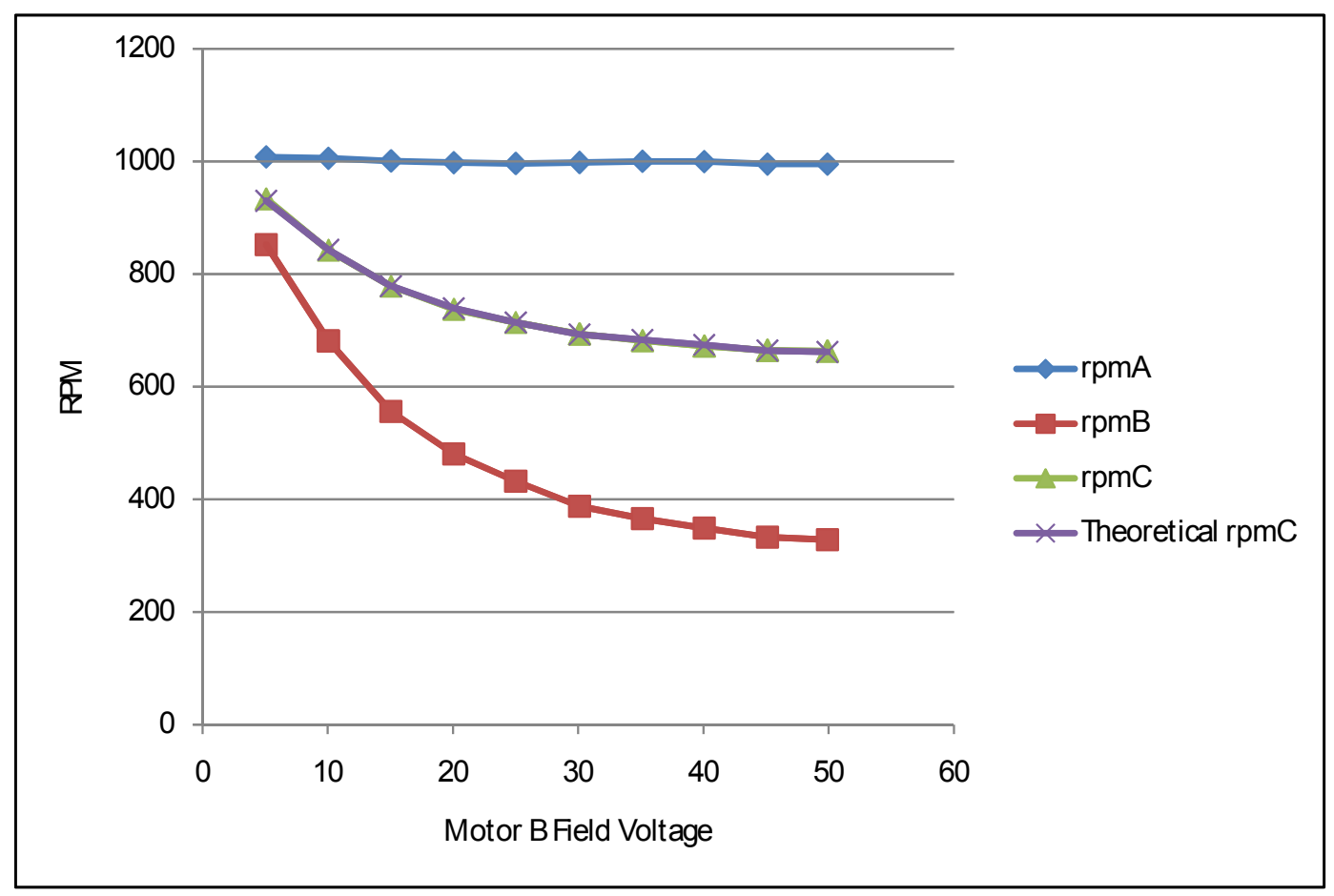

Figure 4.3: RPM Output Verification - Field Variation 


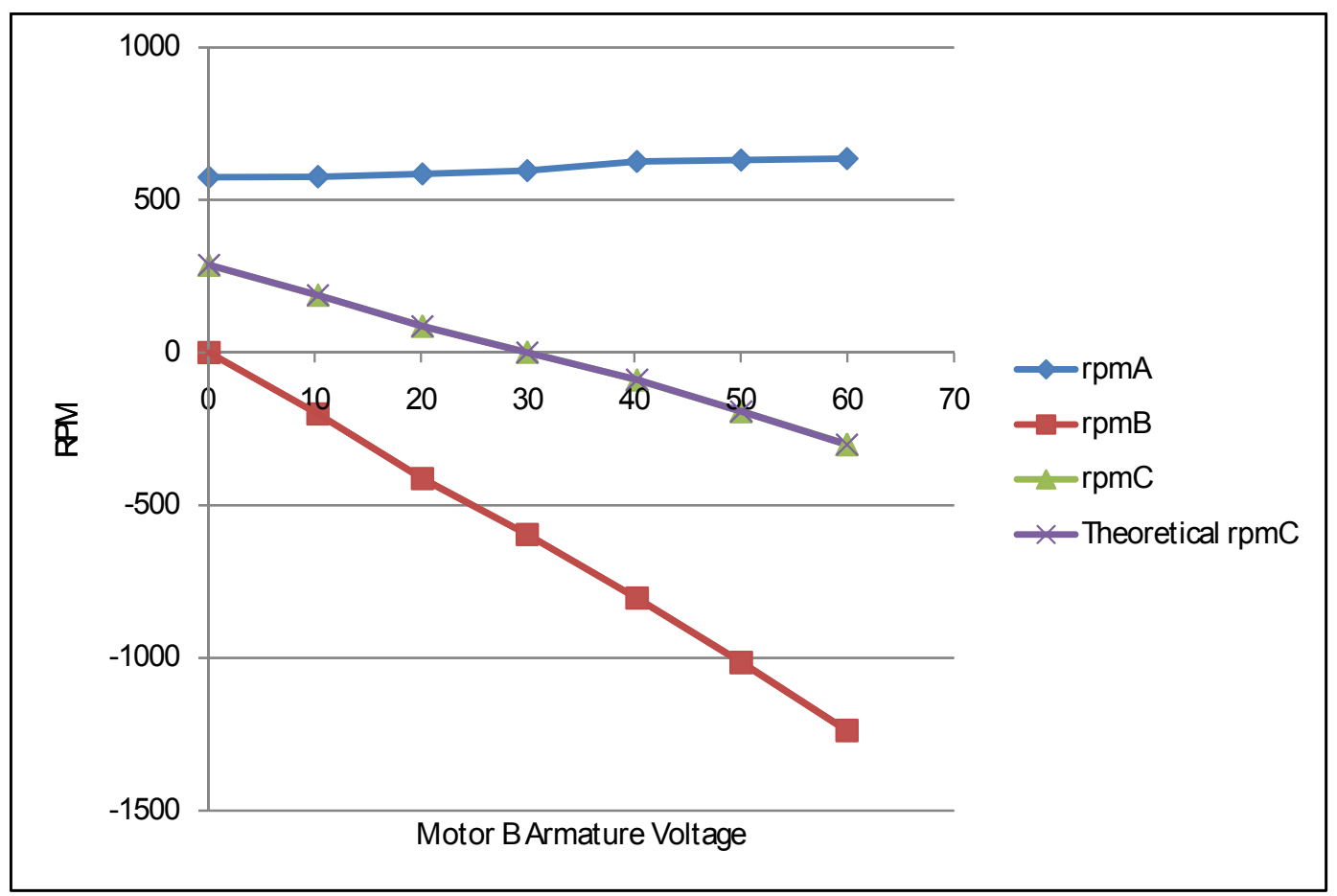

Figure 4.2: RPM Output Verification - Armature Variation

Although expected, this confirmation of rpm data substantiates future testing regarding rpm output characteristics. In addition to confirming Equation (3.1), Figure 4.2 also displays that under no load the differential can function as an IVT, meaning it can have a zero rpm output with non-zero inputs. The zero output is indicated at Motor B armature voltage point 30 in Figure 4.2.

For all subsequent tests a numerical value followed by an 's' indicates the test was performed with the inputs in the same direction. Any tests with numerical values followed by an 'o' denotes the inputs were running in opposite directions.

\subsection{Load Test - Inputs In The Same Direction}

\subsubsection{Test $1 \mathrm{~s}$}

In this set of tests the two input motors operated in the positive direction. As outlined in the test procedure in Chapter 3, data were taken at various armature voltage settings for Motor B. For this test the field voltage on Motor B was held constant. The voltages on the armature and field 
of Motor A were also held constant. Table 4.1 displays the armature voltage settings for Motors A and B. Figure 4.4 and 4.5 show the system rpm and torque inputs and output.

Table 4.1: Test 1s - Motor A and B Armature Voltage Values

\begin{tabular}{|l|c|c|c|c|c|c|}
\hline & \multicolumn{6}{|c|}{ Armature Voltage } \\
\hline Motor A & 50 & 50 & 50 & 50 & 50 & 50 \\
\hline Motor B & 0 & 10 & 20 & 30 & 40 & 50 \\
\hline
\end{tabular}

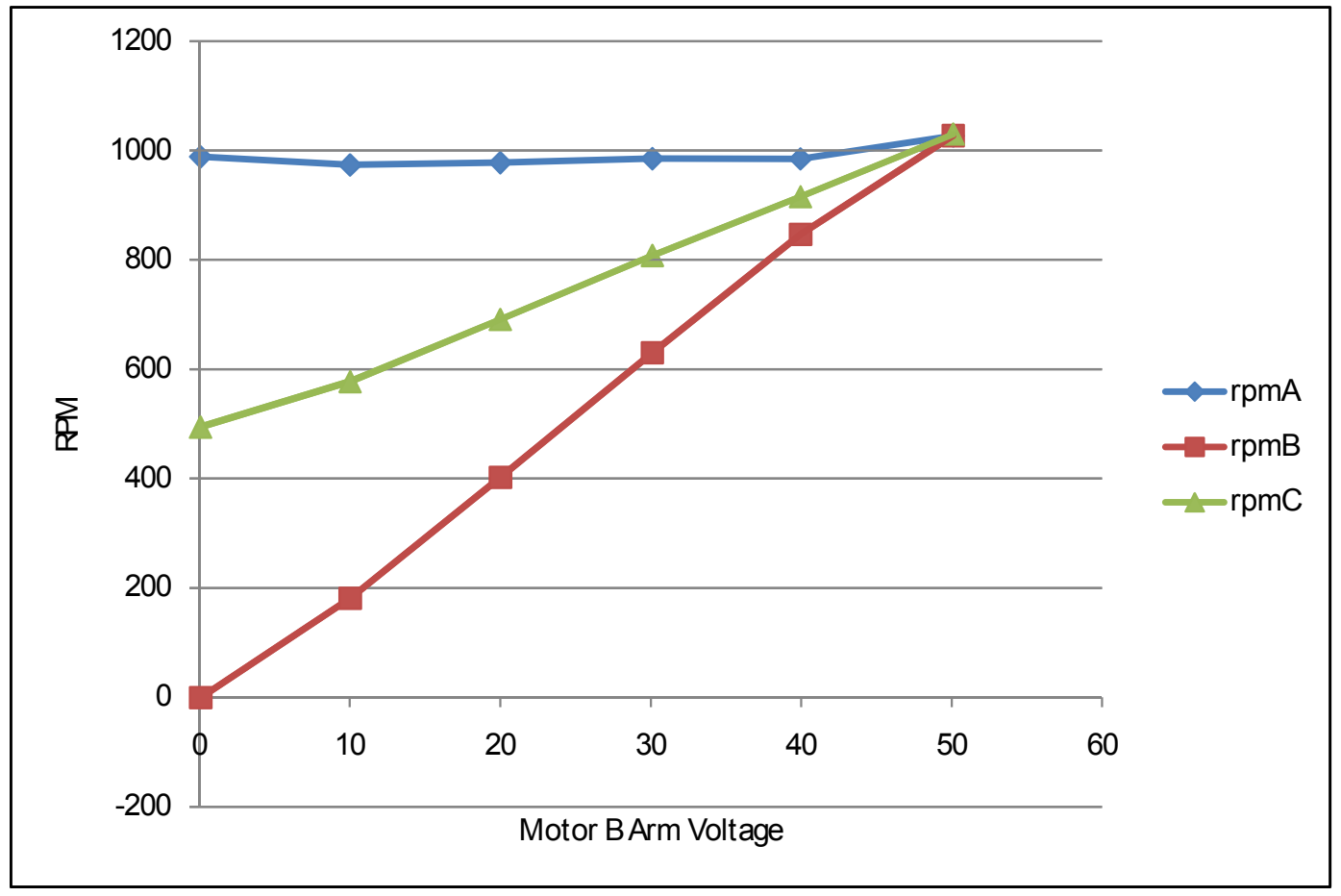

Figure 4.4: Test 1s RPM Output - Same Direction Inputs

Observations for the test data included in Figure 4.4 are:

1. Motor A rpm stays relatively constant at approximately 1000

2. Motor B rpm values increase from 0 to 1000

3. The output rpm, rpmC, values increase from 500 to 1000 


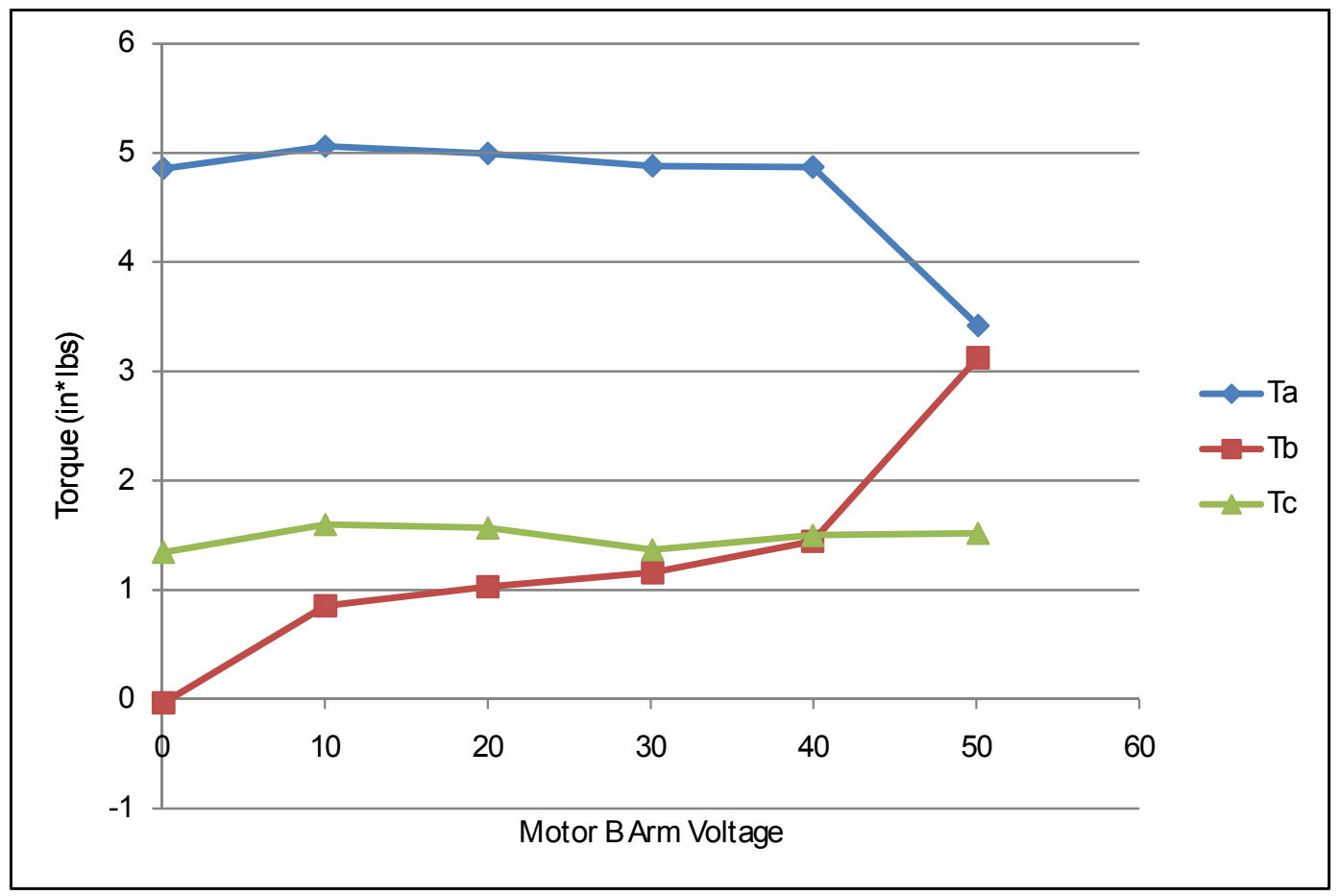

Figure 4.5: Test 1s Torque Output - Same Direction Inputs

Test data in Figure 4.5 provides an indication that:

1. The system was loaded, Tc, at a value of about $1.5 \mathrm{in} \cdot \mathrm{lbs}$

2. Motor A supplied about $5 \mathrm{in} \cdot \mathrm{lbs}$ until Motor B was at the same rpm (see Figure 4.4)

3. Motor B gradually increased torque supply to the system from 0 to $3 \mathrm{in} \cdot \mathrm{lbs}$

4. When Motor A and Motor B were at the same rpm value, both supplied similar amounts of torque to the system

\subsubsection{Test $2 \mathrm{~s}$}

For this set of tests a slightly different approach was taken. Instead of varying only the armature voltage on Motor B, the armature voltage on Motor A was also varied. Table 4.2 shows the armature voltage values at which the data points were collected. Field voltages on both motors remained constant at 50 volts for the duration of each test. Figures 4.6 and 4.7 show the results of 
the tests graphically. Notice that the armature voltage values in Table 4.2 for Motor A are generally double the armature voltage value of Motor B.

Table 4.2: Test 2s - Motor A and B Armature Voltage Values

\begin{tabular}{|c|c|c|c|c|c|c|}
\hline & \multicolumn{5}{|c|}{ Armature Voltage } \\
\hline Motor A & 5 & 10 & 20 & 30 & 40 & 50 \\
\hline Motor B & 0 & 5 & 10 & 15 & 20 & 25 \\
\hline
\end{tabular}

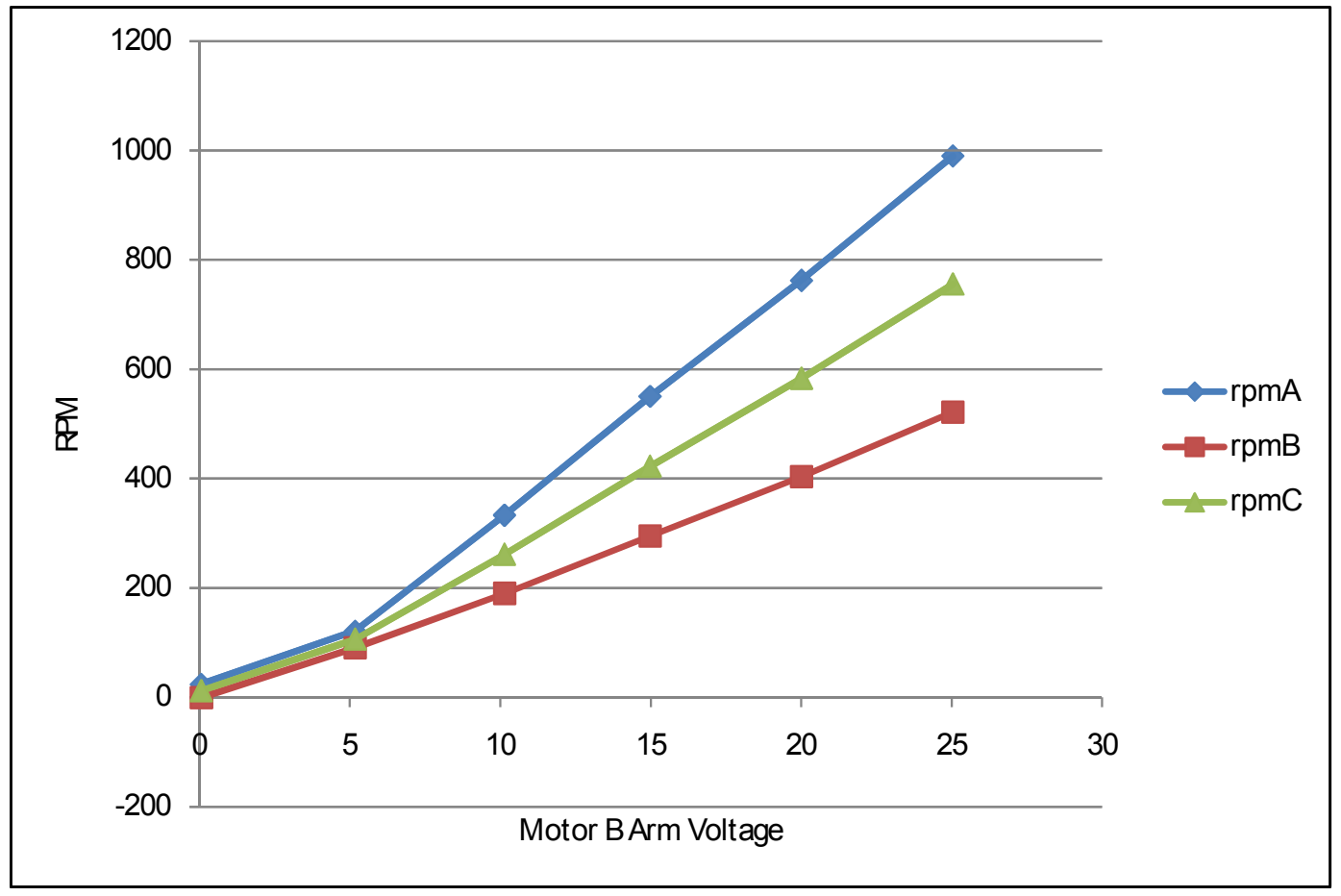

Figure 4.6: Test 2s RPM Output - Same Direction Inputs

The following observations from Figure 4.6 are:

1. Motor A rpm values increase from 0 to 1000

2. Motor B rpm values increase from 0 to 500

3. Output rpmC values increase from 0 to 750 


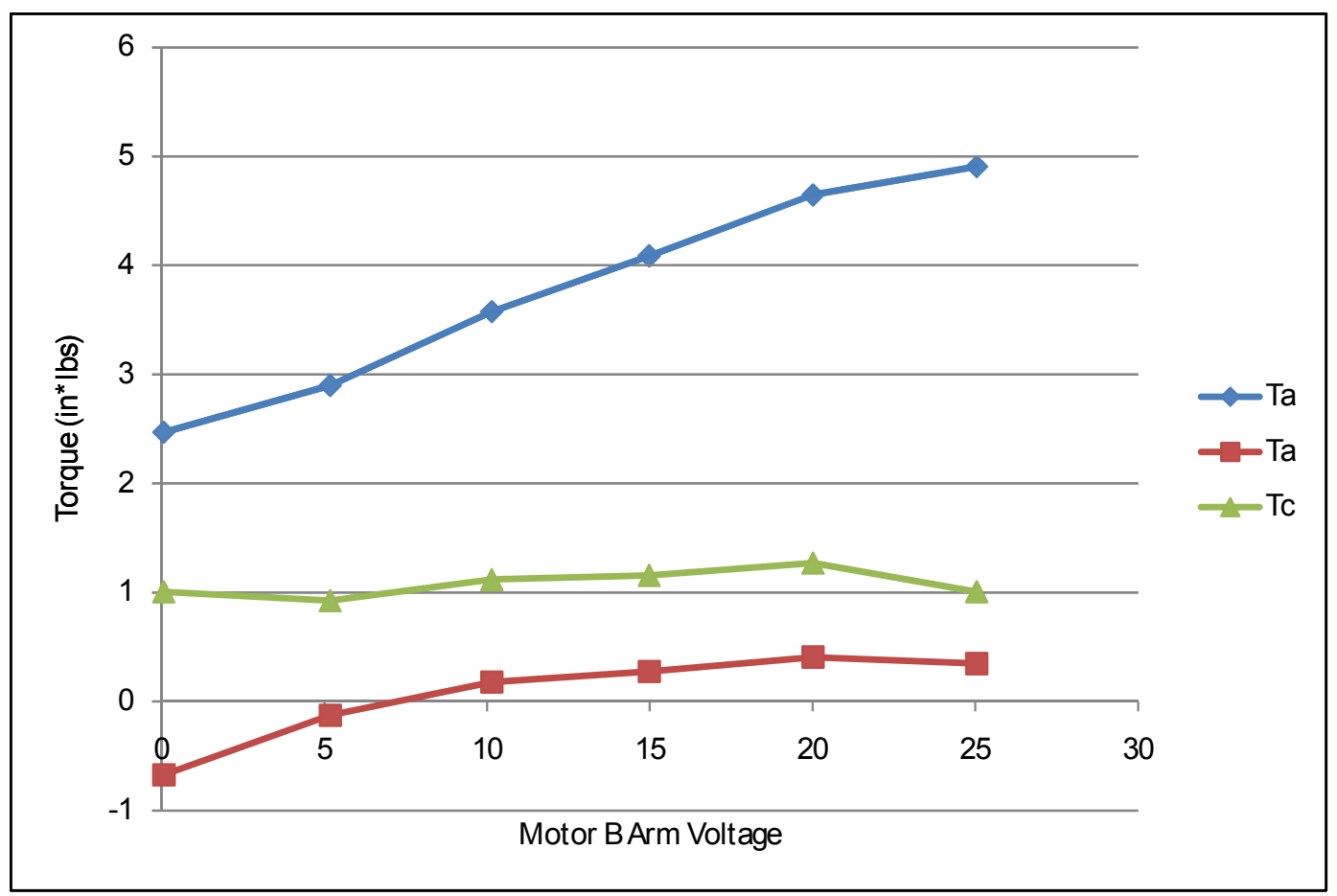

Figure 4.7: Test 2s Torque Output - Same Direction Inputs

Observations from Figure 4.7 include:

1. The system load, Tc, was about $1 \mathrm{in} \cdot \mathrm{lb}$

2. Motor A torque values increase from 2.5 to $5 \mathrm{in} \cdot \mathrm{lbs}$

3. Motor B torque values increase from torque absorbtion at $-0.5 \mathrm{in} \cdot \mathrm{lbs}$ to an input torque of $0.5 \mathrm{in} \cdot \mathrm{lbs}$

\subsection{Load Test - Inputs In Opposite Directions}

\subsubsection{Test 10}

For these tests the system inputs ran in opposite directions. Again, data were taken as the armature voltage on Motor B was varied. The field on Motor B was held constant at 50 volts. The field and armature on Motor A were held constant at 50 volts. Table 4.3 shows the armature voltage values for Motor A and Motor B. Figures 4.8 and 4.9 show each Motor's rpm and torque 
input as well as the system rpm and torque output. Note that the incremental voltage value for the armature of Motor B is 10 volts.

Table 4.3: Test 1o - Motor A and B Armature Voltage Values

\begin{tabular}{|l|c|c|c|c|c|c|c|}
\hline & \multicolumn{7}{|c|}{ Armature Voltage } \\
\hline Motor A & 50 & 50 & 50 & 50 & 50 & 50 & 50 \\
\hline Motor B & 0 & 10 & 20 & 30 & 40 & 45 & 50 \\
\hline
\end{tabular}

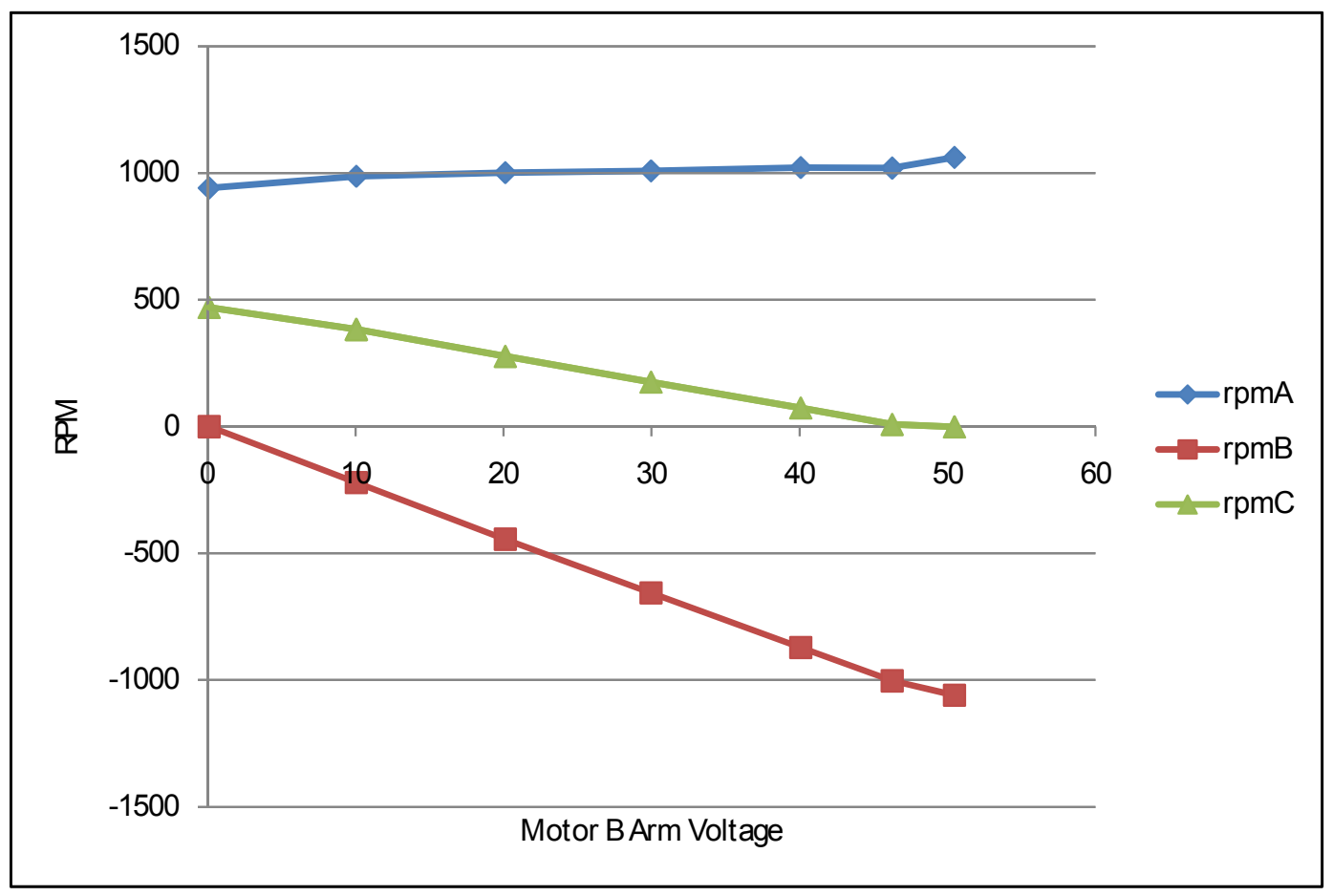

Figure 4.8: Test 1o RPM Output - Opposite Direction Inputs

Observations from Figure 4.8 include:

1. The rpm of Motor A stays fairly constant at about 1000

2. Motor $\mathrm{B}$ rpm values decrease from 0 to -1000

3. The output rpm, rpmC, decreases from 500 to 0 


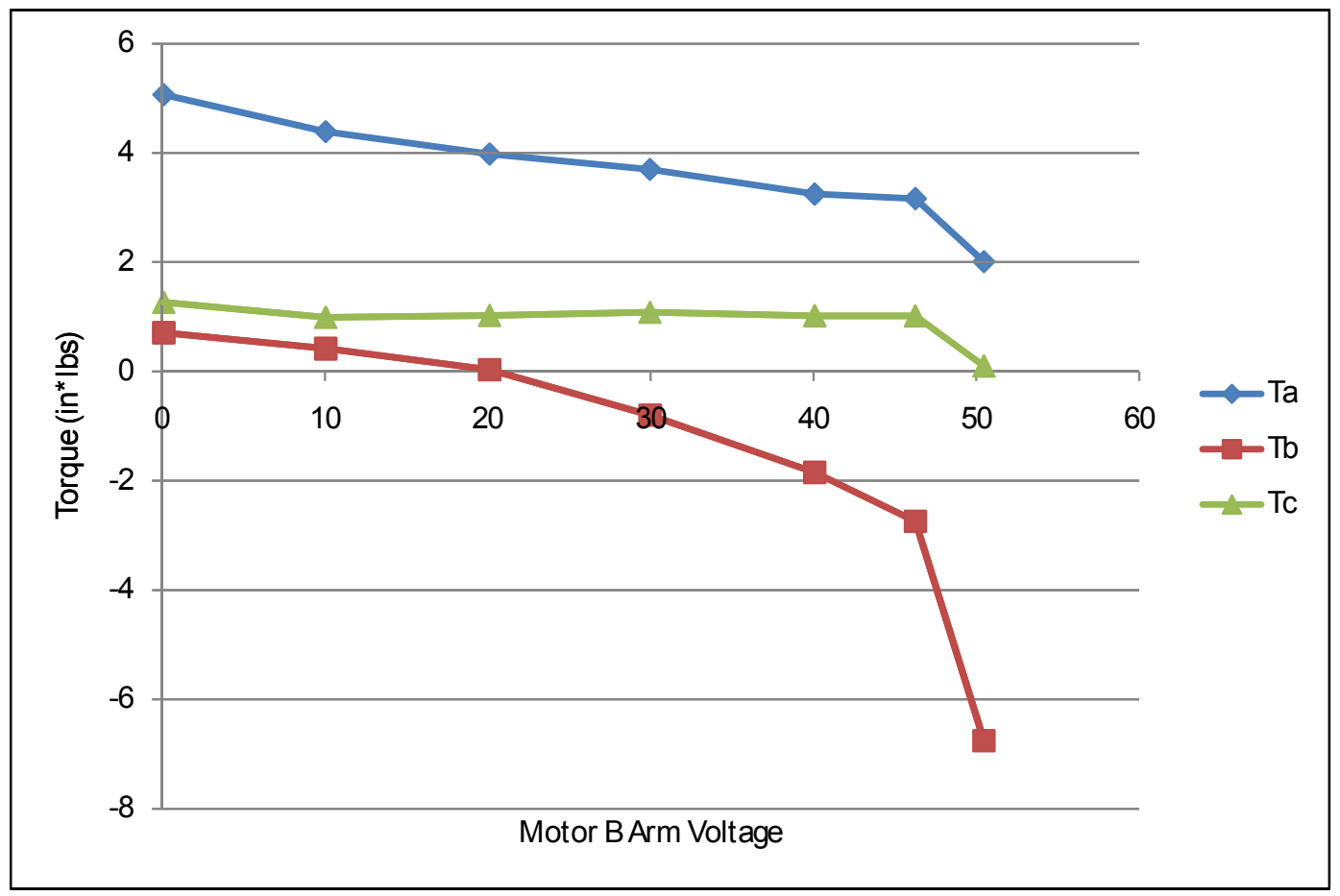

Figure 4.9: Test 1o Torque Output - Opposite Direction Inputs

Inspection of Figure 4.9 gives the following observations:

1. System was loaded at around $1 \mathrm{in} \cdot \mathrm{lb}$

2. Motor A supplied descending torque values from 5 to $2 \mathrm{in} \cdot \mathrm{lbs}$

3. Motor B initially absorbed torque from the system when it had rpm values between 0 and $-500$

4. Motor B supplied torque from 0 to $-2.5 \mathrm{in} \cdot \mathrm{lbs}$ when it had rpm values between -500 and $-1000$

5. Motor B supplied $-7 \mathrm{in} \cdot \mathrm{lbs}$ of torque at $1,100 \mathrm{rpm}$

6. As Motor B supplied more torque to the system, Motor A supplied less torque to the system

7. When the output rpm value was zero, at 50 volts, no load could be applied, but significant torque values from both motors were still supplied to the system 


\subsubsection{Test 20}

As in the preceding test $2 \mathrm{~s}$, this test varied the armature voltage to both motors and held the field voltages constant at 50 volts. Table 4.4 shows the armature voltage values used. Figures 4.10 and 4.11 show the results.

Table 4.4: Test 2o - Motor A and B Armature Voltage Values

\begin{tabular}{|c|c|c|c|c|c|c|}
\hline & \multicolumn{5}{|c|}{ Armature Voltage } \\
\hline Motor A & 5 & 10 & 20 & 30 & 40 & 50 \\
\hline Motor B & 0 & 5 & 10 & 15 & 20 & 25 \\
\hline
\end{tabular}

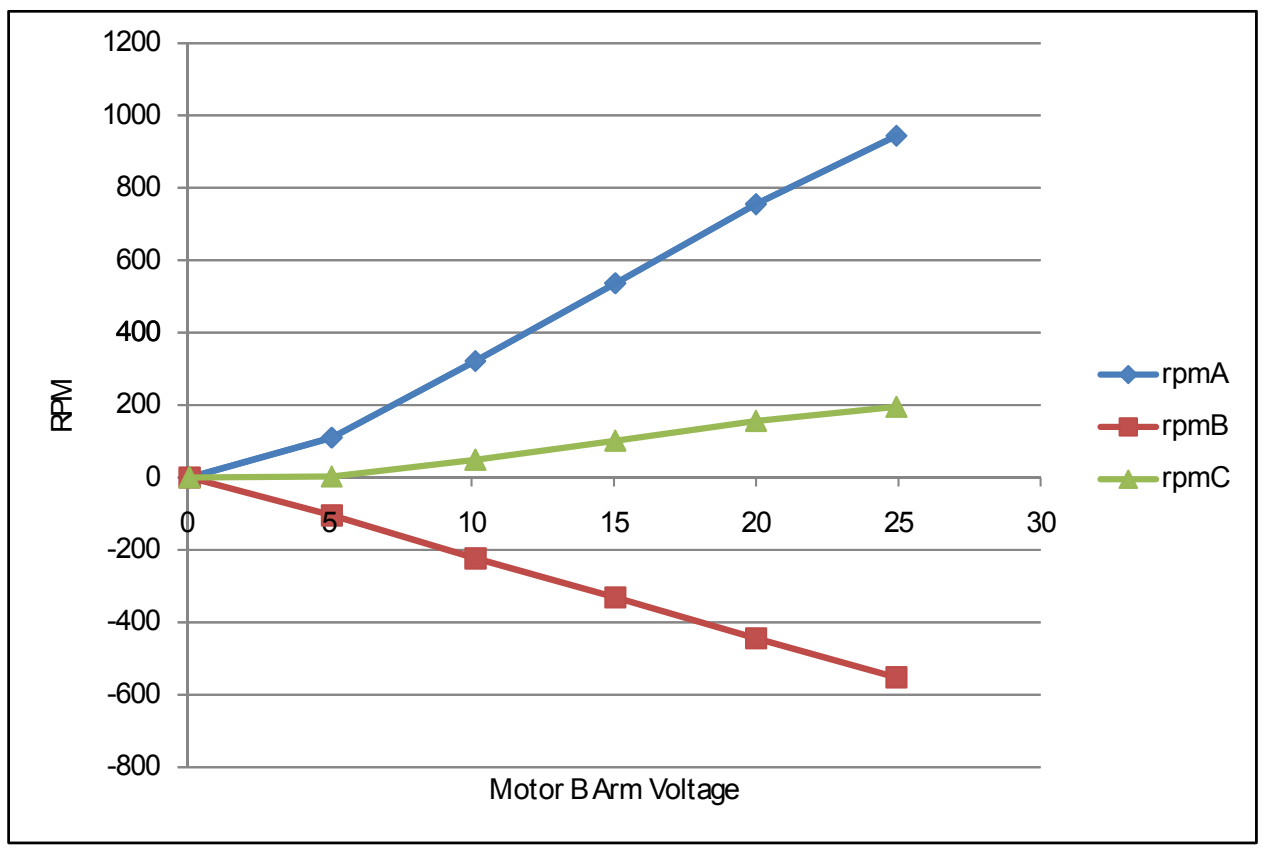

Figure 4.10: Test 2o RPM Output - Opposite Direction Inputs

Observations from Figure 4.10 include:

1. Motor A rpm values increase from 0 to 950

2. Motor B rpm values increase in the opposite direction from 0 to -550

3. The output rpm, rpmC, increases from 0 to 200 


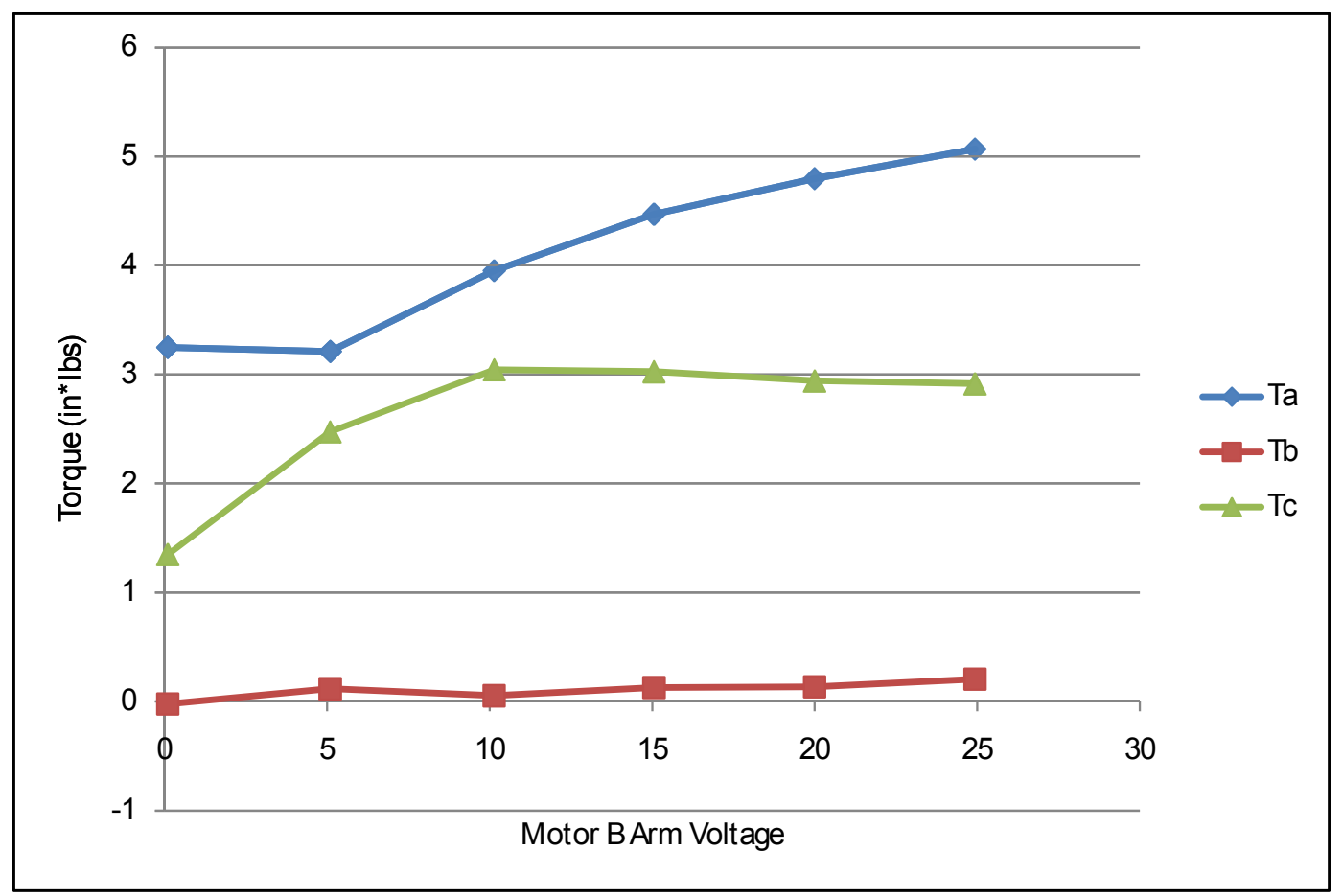

Figure 4.11: Test 2o Torque Output - Opposite Direction Inputs

Examination of Figure 4.11 indicates that:

1. Loading of the system was from 1.5 to $3 \mathrm{in} \cdot \mathrm{lbs}$

2. Motor A provided 3.25 to $5 \mathrm{in} \cdot \mathrm{lbs}$ of torque to the system

3. Motor B provided negligible torque to the system

\subsection{Load Test - Cross Over}

This test incorporated a polarity change of the Motor B to see what characteristics would be displayed by the differential. Table 4.5 shows the armature voltages for the motors for this test. The fields on both motors were held constant at 50 volts. Figures 4.12 and 4.13 display the results of the test. 
Table 4.5: Cross Over Armature Voltage Values

\begin{tabular}{|l|c|c|c|c|c|c|}
\hline & \multicolumn{6}{|c|}{ Armature Voltage } \\
\hline Motor A & 30 & 30 & 30 & 30 & 30 & 30 \\
\hline Motor B & -60 & -50 & -40 & -30 & -20 & -10 \\
\hline \hline Motor A & 30 & 30 & 30 & 30 & 30 & 30 \\
\hline Motor B & 0 & 10 & 20 & 30 & 40 & 50 \\
\hline
\end{tabular}

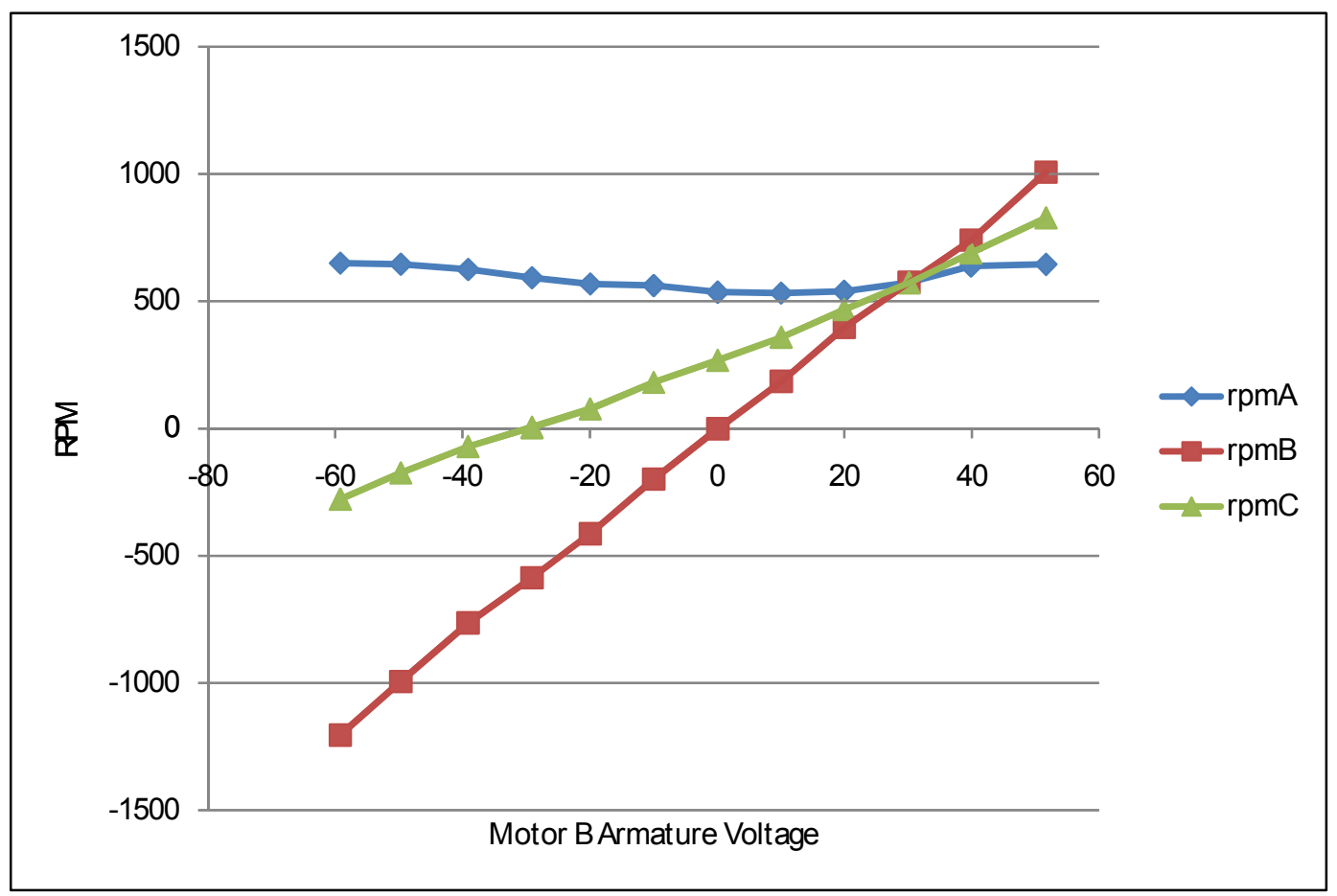

Figure 4.12: Cross Over RPM Output

Observations from this test include:

1. Motor A rpm values stayed relatively constant at about 600

2. Motor $B$ rpm values varied from -1200 to 1000

3. Output rpm, rpmC, varied from -250 to 1000

4. Output rpm, rpmC, had a zero output value at the -30 volt mark 


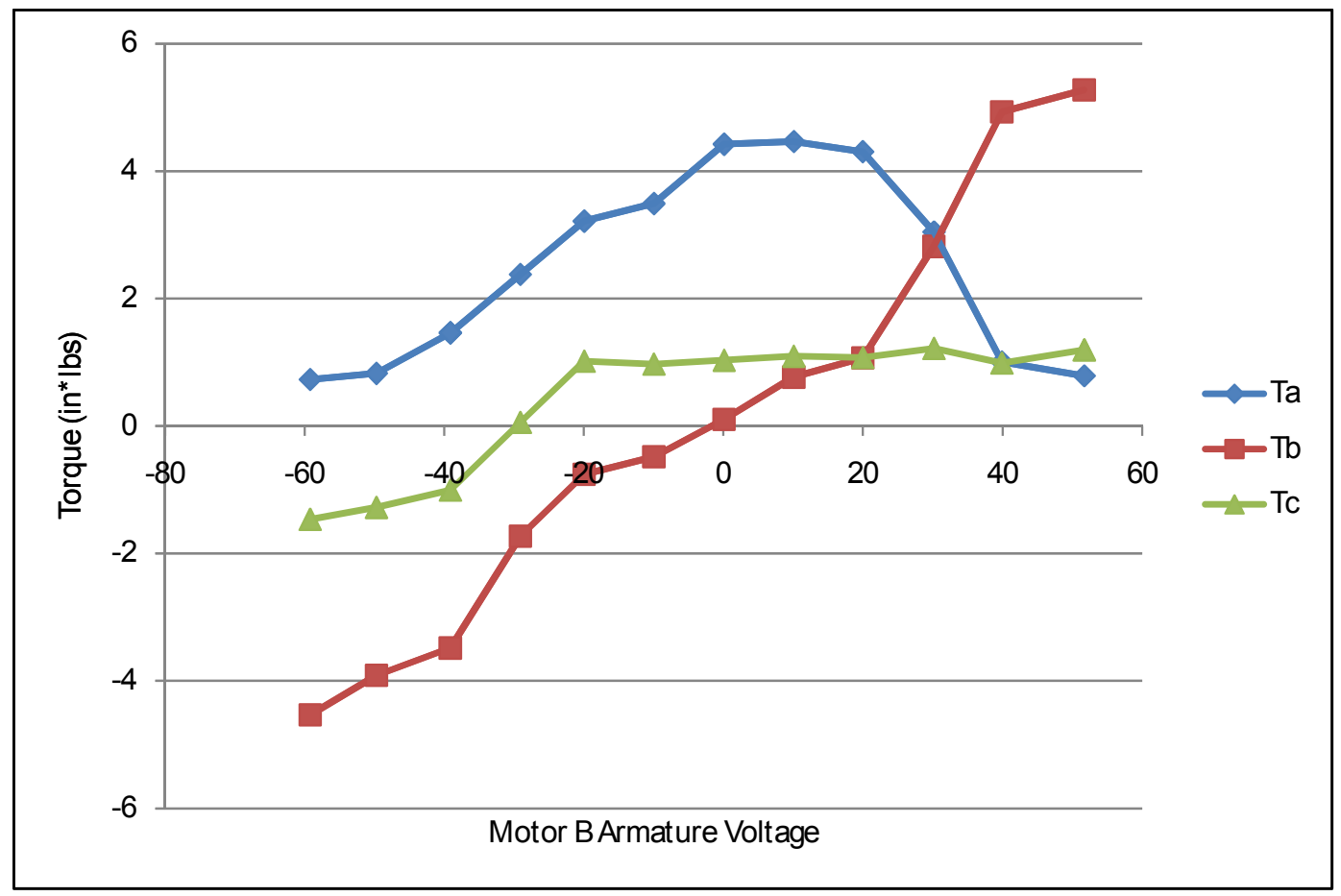

Figure 4.13: Cross Over Torque Output

The data in Figure 4.13 indicates that:

1. The output torque, Tc, was initially in the negative direction at about $1 \mathrm{in} \cdot \mathrm{lb}$ and then went in the positive direction at $1 \mathrm{in} \cdot \mathrm{lb}$

2. Motor A supplied torque to the system varying between 1 and $4.25 \mathrm{in} \cdot \mathrm{lbs}$

3. Motor B supplied torque to the system between the values of -4.5 and $5 \mathrm{in} \cdot \mathrm{lbs}$

4. Motor A and Motor B supplied equal torque to the system when they exhibited equal rpm in the same direction (30 volt mark)

5. Motor A and Motor B supplied similar torque to the system when they exhibited equal rpm in opposite directions (-30 volt mark) 


\section{CHAPTER 5. DISCUSSION OF RESULTS}

\subsection{RPM Verification}

As discussed earlier, the theoretical Equation (4.1) was proven valid for rpm prediction at the output rpmC. The physical meshing of the gears within the differential makes this prediction possible. Also, Figure 4.2 verified that a differential, with no applied load, could be used as an IVT (have zero rotational output with non-zero inputs). The zero rotational output can be seen at the 30 volt mark on the horizontal axis in Figure 5.1.

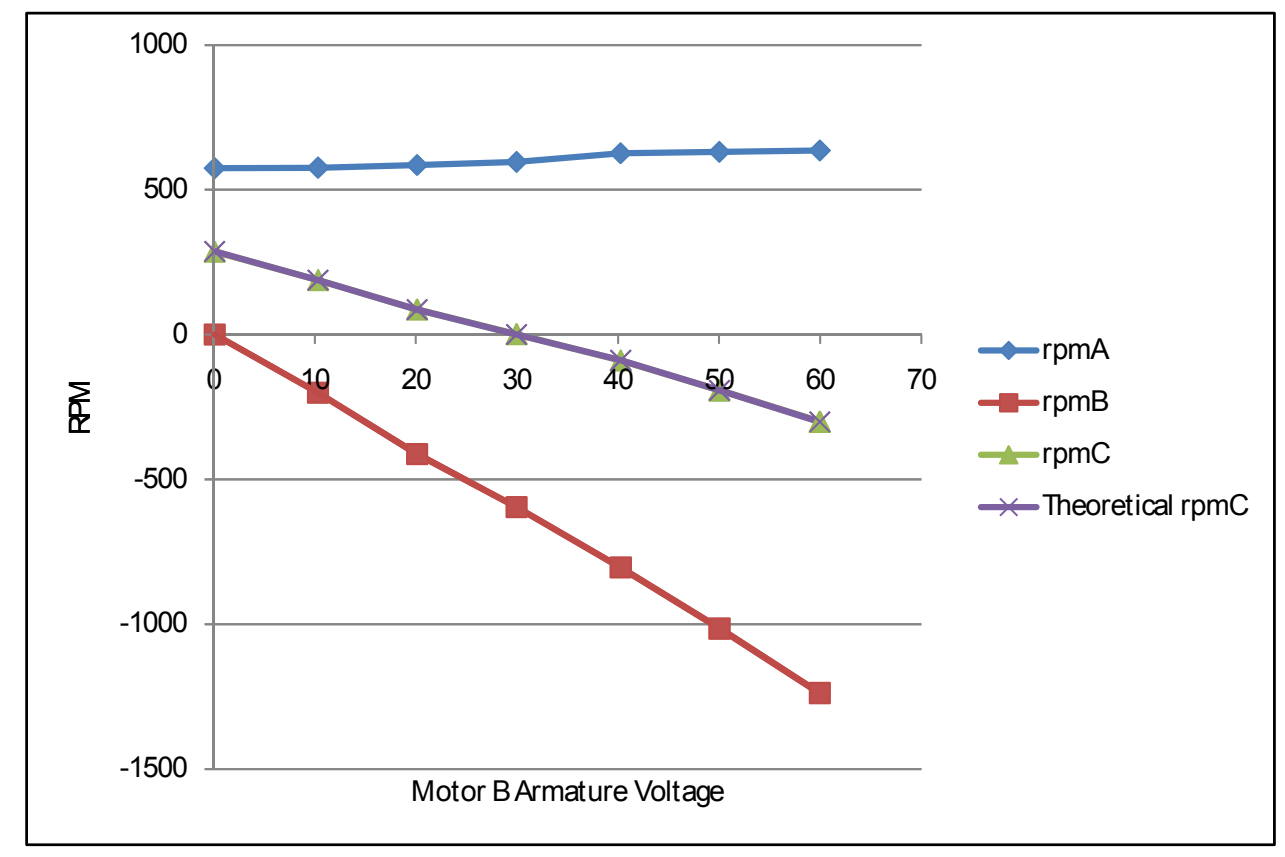

Figure 5.1: RPM Output Verification - Armature Variation: same as Figure 4.2

For a hybrid vehicle application a point of zero output would be important for instances when the vehicle is in a stopped position, even though the inputs may still be running and providing rotational input to the system. 


\subsection{Load Test - Inputs In The Same Direction}

\subsubsection{Load Test 1s}

Load test $1 \mathrm{~s}$ showed that while supporting a load the output could accelerate or decelerate from one angular velocity to another. This would be advantageous in a hybrid vehicle application if one input was to remain at a constant angular velocity, for efficiency purposes, while the other input accelerated to provide accelerated output (vehicle acceleration) from one velocity to a higher velocity. This would be applicable in cases such as in passing another vehicle. Figure 5.2 displays how the differential functions as a CVT and allows the output, rpmC, to change continuously within a certain range.

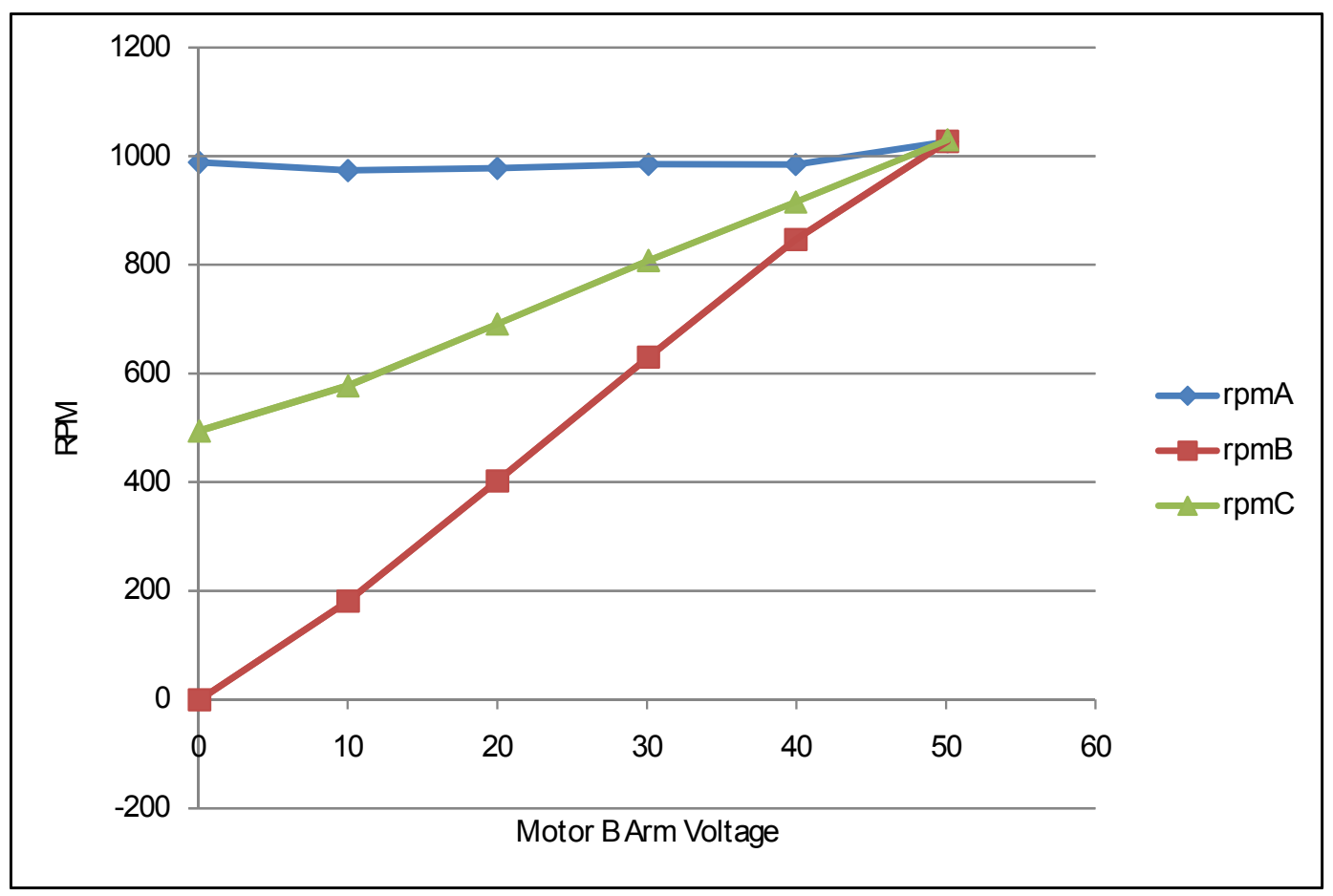

Figure 5.2: Test 1s RPM Output - Same Direction Inputs: same as Figure 4.4

Another interesting insight gained from this experiment is that as Input B approached Input A's angular velocity the inputs shared the load more equally, see Figure 5.3. 


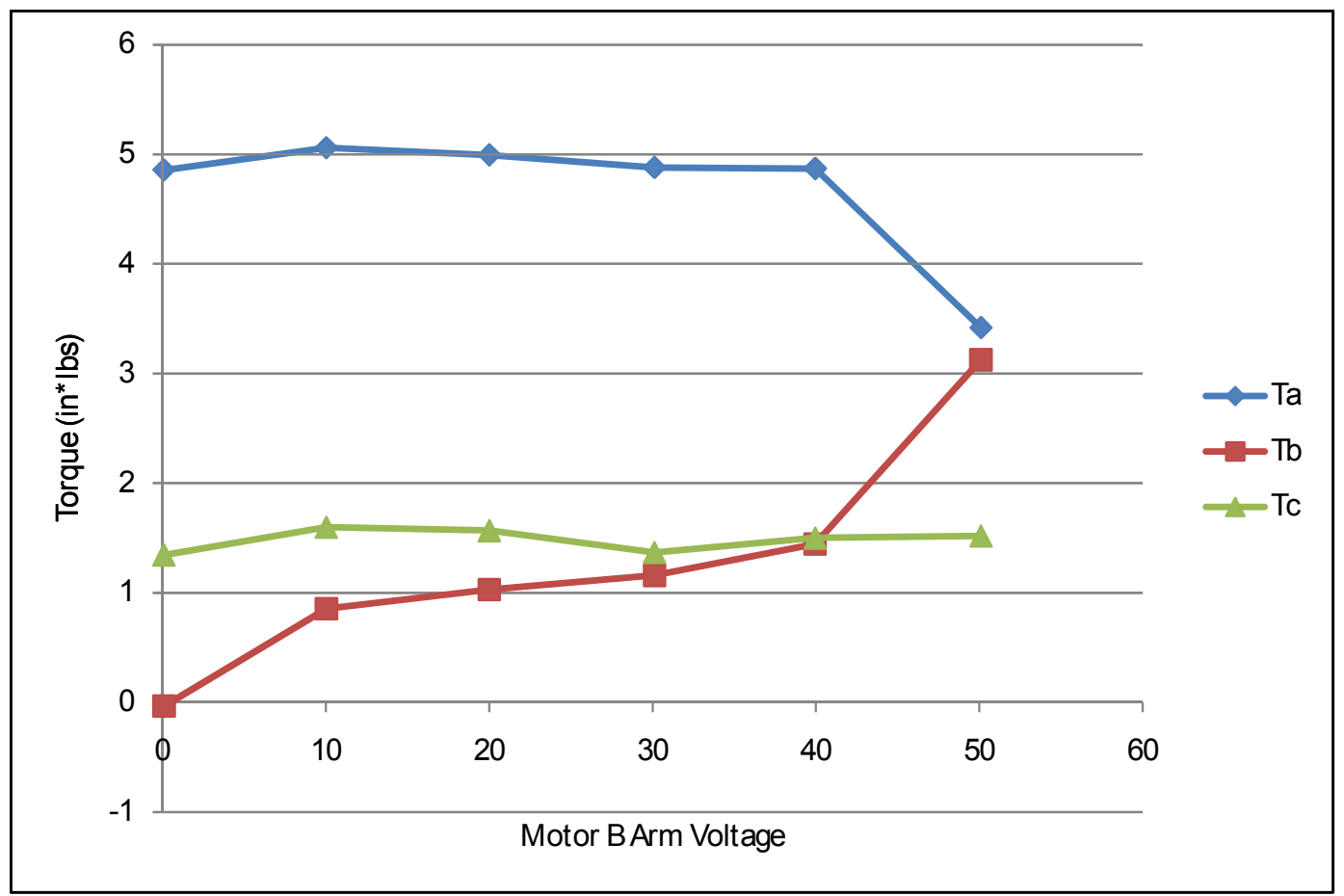

Figure 5.3: Test 1s Torque Output - Same Direction Inputs: same as Figure 4.5

In a vehicle application it may be desired that one input, for example Input A, carry the majority of the load. If it is desired that Input A carry a larger portion of the load, additional gear reduction design could be incorporated into Input B to facilitate a torque/rpm tradeoff. Meaning, Input B could revolve at a higher angular velocity but supply less torque to the differential input shaft via a gear reduction to the Input B differential shaft. This will be explored in greater detail later in this chapter.

\subsubsection{Load Test $2 \mathrm{~s}$}

Load test $2 \mathrm{~s}$ displayed results that indicated an output under load could increase from a zero angular velocity while Input A carried substantially more of the load than Input B. Under these conditions a vehicle could accelerate from a stopped position and have Input A carry the bulk of the load during acceleration. See Figures 5.4 and 5.5. 


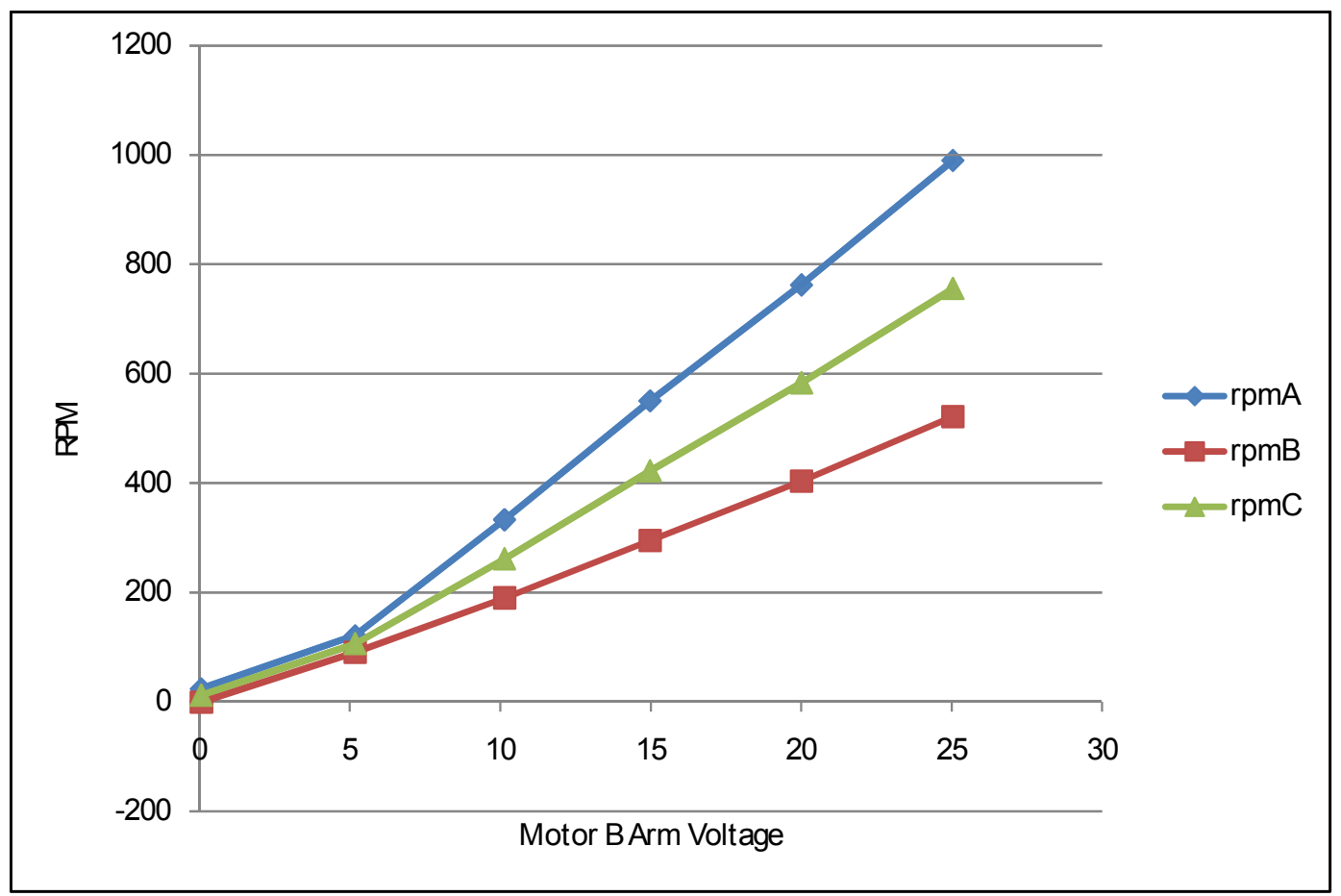

Figure 5.4: Test 2s RPM Output - Same Direction Inputs: same as Figure 4.6

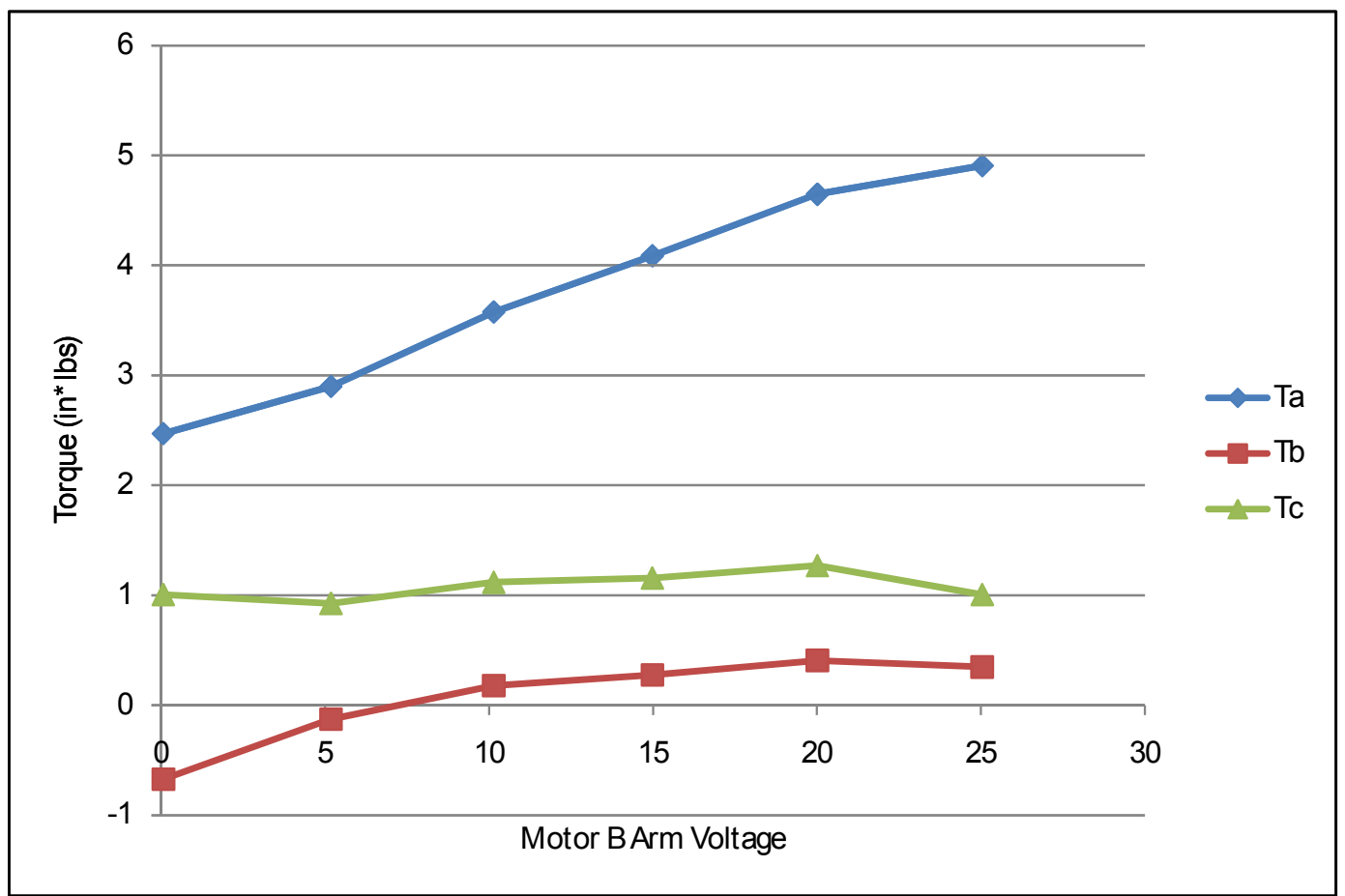

Figure 5.5: Test 2s Torque Output - Same Direction Inputs: same as Figure 4.7 


\subsection{Load Test - Inputs In Opposite Directions}

\subsubsection{Load Test 1o}

Test 10 showed that when the inputs are in opposite directions the dual-input, single-output differential system can support a load. However, this test also proved that common differentials are backdrivable. Being backrivable means that the torques from each input are not independent of one another and that the torque flow path will not look like the one shown in Figure 5.6.

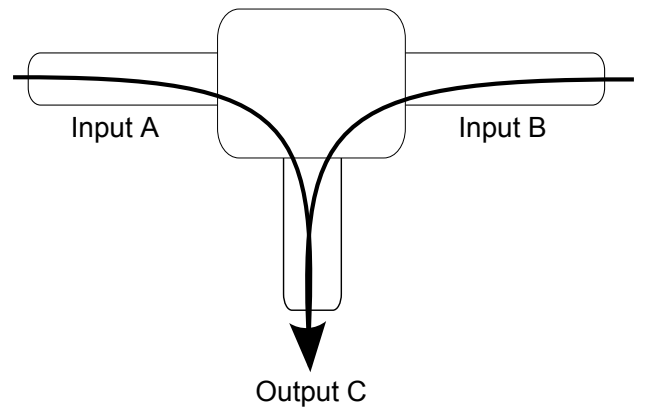

Figure 5.6: Torque Flow of a Dual-input Non-backdrivable Differential: same as Figure 3.5

In actuality, the torque flow will find the path of least resistance and regularly translate from one input to the other input when the load on the output is large. This translation of torque, or back drive, causes a regenerative effect on the input providing the least amount of torque to the system, as in Figure 5.7.

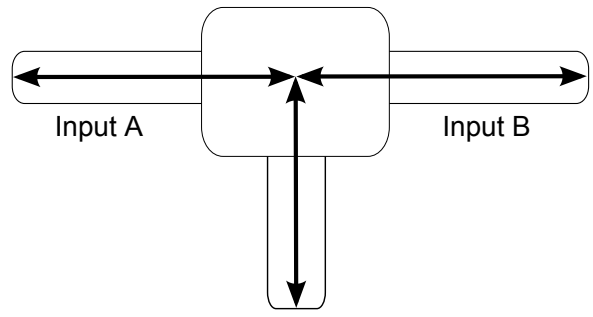

Output C

Figure 5.7: Torque Flow of a Dual-input Backdrivable Differential 
Figure 5.7 indicates that each differential shaft or disc described thus far in this work is actually an input and an output, depending on the load on Output C. Hence, each shaft or disc could also be called an input/output or an I/O. For consistency, the remainder of this work will continue to reference each $\mathrm{I} / \mathrm{O}$ as has been denoted previous to this discovery (i.e. Input A, Input B, and Output C).

When the load on Output $\mathrm{C}$ is large backdriving occurs and torque is transferred from one input to the other, creating a generative effect instead of an input effect. Figure 5.8 shows that in theory, for a non-backdrivable differential, as Input B's rpm value approaches Input A's rpm value in the opposite direction Output rpmC should approach zero as the torque for Output C approaches infinity. As can be seen in Figures 5.9 and 5.10 this is not the case.

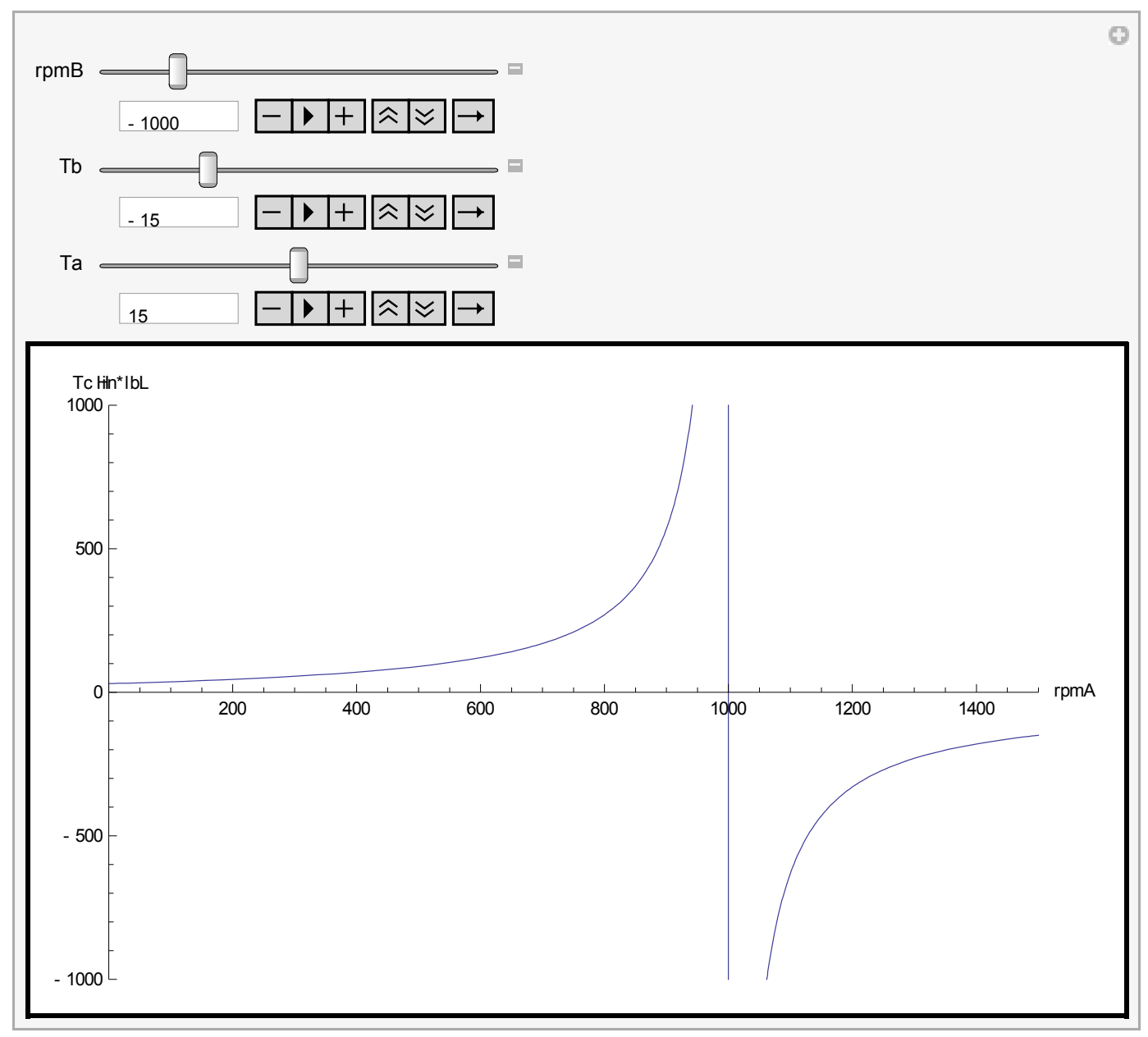

Figure 5.8: Graphical Representation of Equation (3.4): same as Figure 3.6 


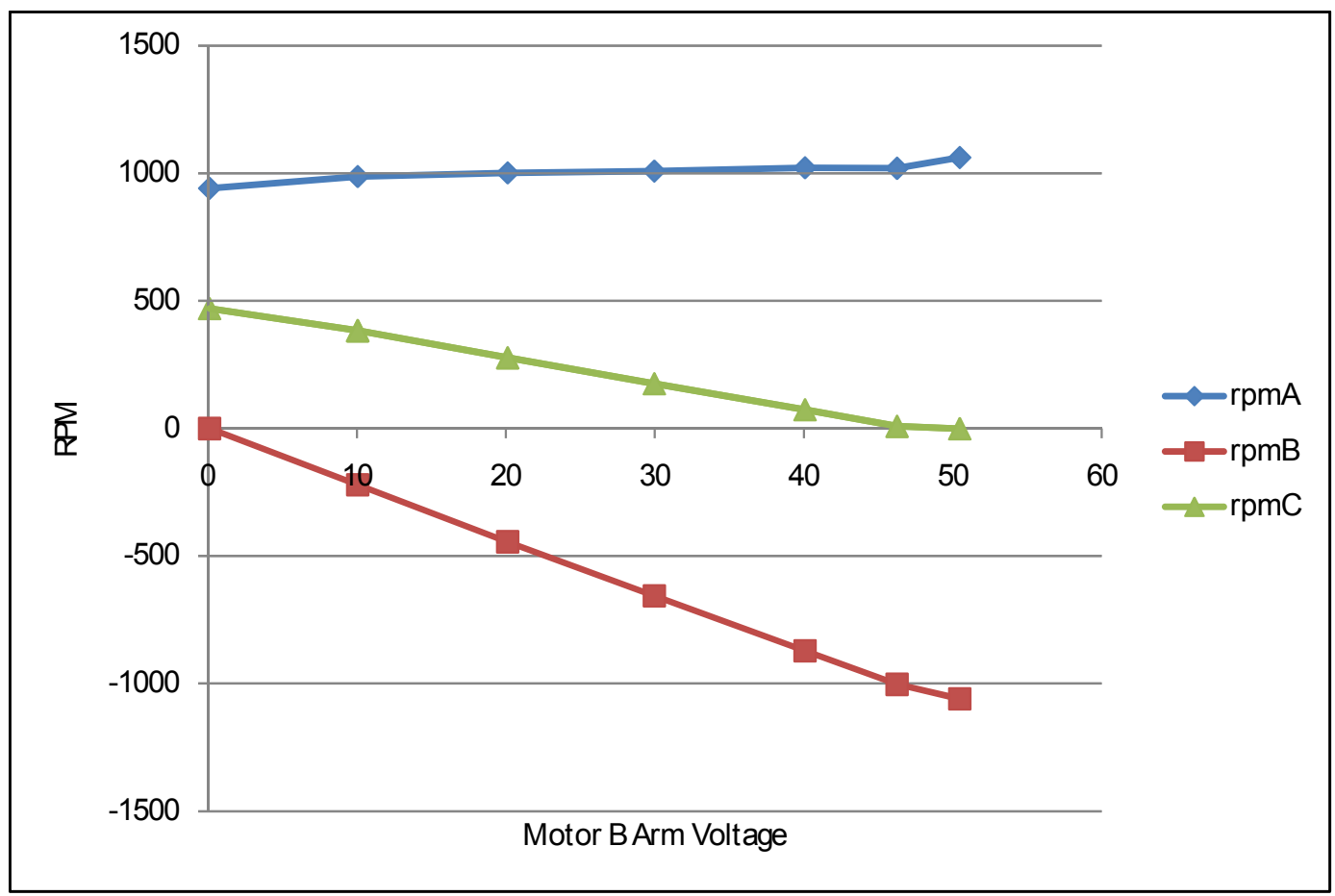

Figure 5.9: Test 1o RPM Output - Opposite Direction Inputs: same as Figure 4.8

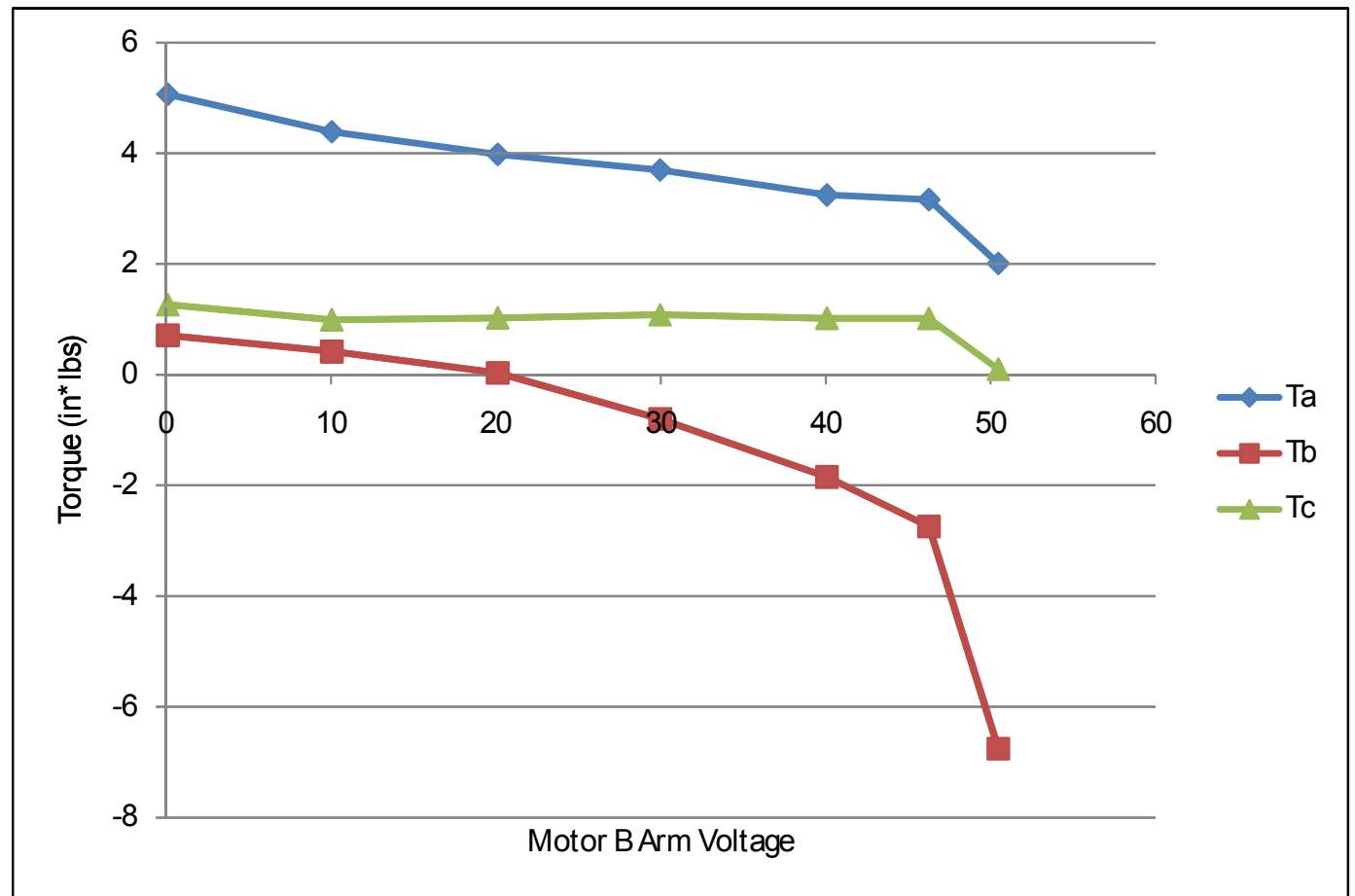

Figure 5.10: Test 1o Torque Output - Opposite Direction Inputs: same as Figure 4.9 
These test results show that as rpmC approaches zero the torque also approaches zero. As previously mentioned, this is because the torque from Input A follows the path of least resistance. As the load, or resistance, increases on Output $\mathrm{C}$ the torque from Input $\mathrm{A}$ tends to be directed toward Input B because the path to Input B is not as difficult as the path to Output C. This indicates that a differential is a torque equalizing device between the two inputs.

Nevertheless, it is thought that if the resistance increases at Input B so that it is higher than the resistance of the load at Output $\mathrm{C}$, the energy would redirect itself toward the output. Increasing the resistance of torque flow to Input B might be possible through gear reduction from the Input B shaft to Motor B. Although this test demonstrated that common differentials are backdrivable it also illustrated that a differential can support a load as the output decelerates from an angular velocity to zero.

\subsubsection{Load Test 2o}

Figures 5.11 and 5.12 magnify the ability of the experimental differential to isolate one input as the load carrier for the system. This can be seen clearly in the previously mentioned figures as almost all of the torque supplied to the system is drawn from Input A while Input B's torque contribution is negligible. This is in conjunction with an increasing angular velocity on Output C, as shown in Figure 5.11.

Recall that the following figures are arranged in such a way that one can view the rpm value of each input or output in conjunction with the corresponding torque value. Color coordination has been implemented to more easily identify the relationships that exist between rpm and torque for the several inputs or output. Understanding the experimental results through the use of the analyzed data portrayed in the figures facilitates the ability to draw accurate conclusions. Being able to view rpm and torque test results, side by side, is fundamental to identifying differential properties pertaining to multiple-input, single-output use. The relationship between rpm and torque in a multiple-input, single-output device, such as a differential, is vital to providing the correct parameters for power source sizing in vehicle powertrain design. 


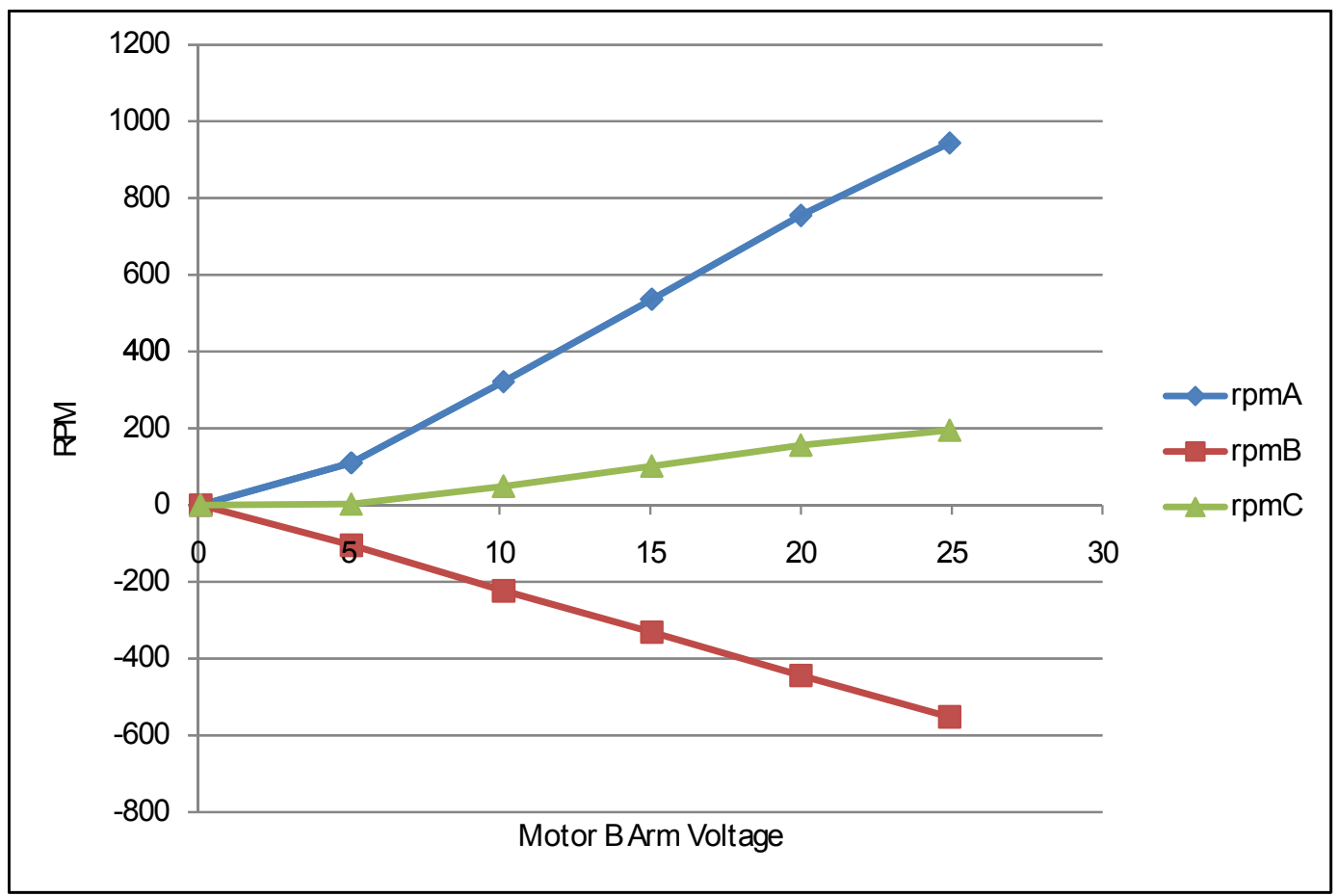

Figure 5.11: Test 2o RPM Output - Opposite Direction Inputs: same as Figure 4.10

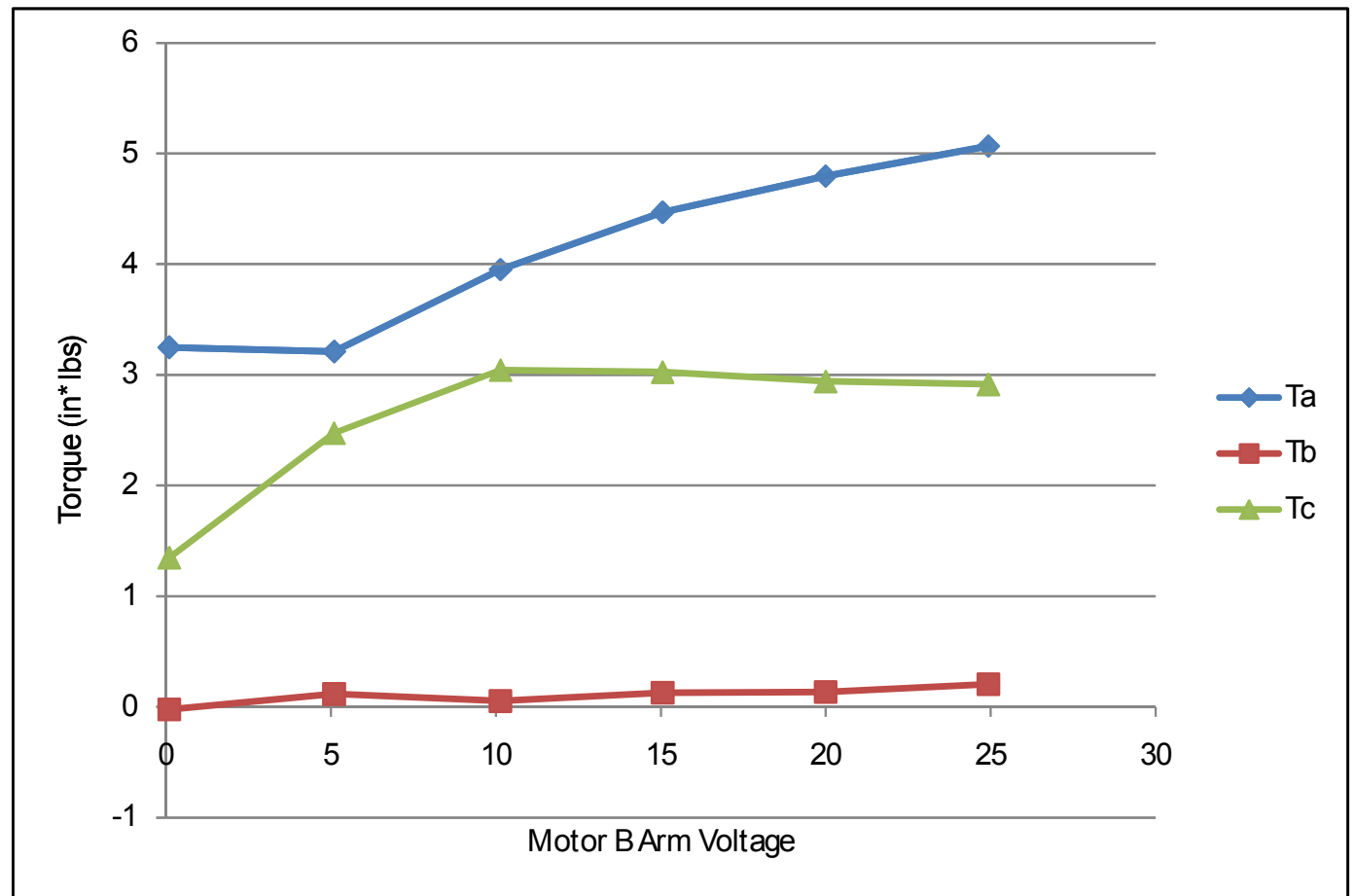

Figure 5.12: Test 2o Torque Output - Opposite Direction Inputs: same as Figure 4.11 
As previously stated, the implication is that a vehicle could start from rest with both inputs providing no rotational input to the differential system, then as the driver pushes on the accelerator the inputs begin to provide rpm increases to the input shafts in opposite directions. As the output shaft begins to accelerate, Input A carries the load and Input B assists in governing the output angular velocity of $\mathrm{C}$. This could be advantageous in a hybrid application as it is usually preferred that the ICE provide the torque to the wheels while the other input assists with only output angular velocity changes to govern vehicle speed. Keeping the output angular velocity lower than the ICE (Input A) allows the ICE to reach the optimal operating range faster and create fuel savings during acceleration. As discussed in Chapter 1, the more time an ICE can spend in an optimal operating range the more torque and power it can provide to the system with the least amount of fuel consumption.

It is important to note that the preceding results in load test 10 indicated that a differential is an input torque equalization device. Here, in load test 20 , it appears that this is not the case because Input A carries the load while Input B carries almost no load. The factor that is most responsible for the imbalance of torque between the inputs is the helical gears in the experimental differential. Helical gears have a high coefficient of friction at low angular velocities, thus allowing Input A to carry more load than Input B. The helical gears in combination with the incremental angular velocity increase, as in load test $2 \mathrm{~s}$ and $2 \mathrm{o}$, seem to combine to allow one input to carry more load than the other. It is probable that using bevel gears, as in most differentials, would negate the ability of the differential to distribute the load unequally between the inputs.

\subsection{Load Test - Cross Over}

The cross-over test demonstrated that a differential can function as an IVT under a load. Here the term cross-over was used to indicate that the polarity (rotational direction) of Input B was changed during the test from a rotation in the direction opposite of Input A to the same direction as Input A. The polarity switch occurs at the Motor B armature voltage value of 0 , which is also Motor B's 0 rpm point. See Figures 4.12 and 4.13. 


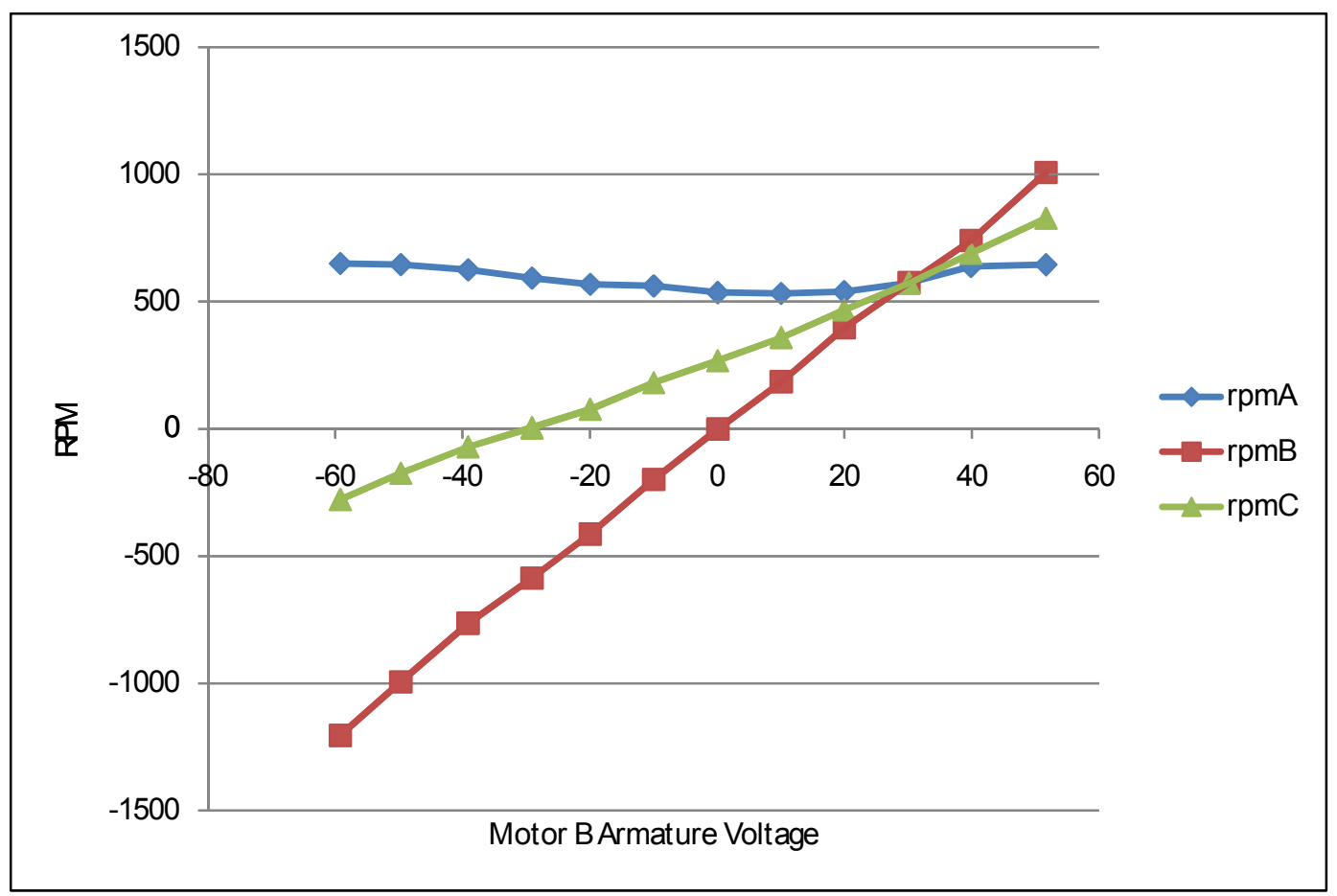

Figure 5.13: Test Cross Over RPM Output: same as Figure 4.12

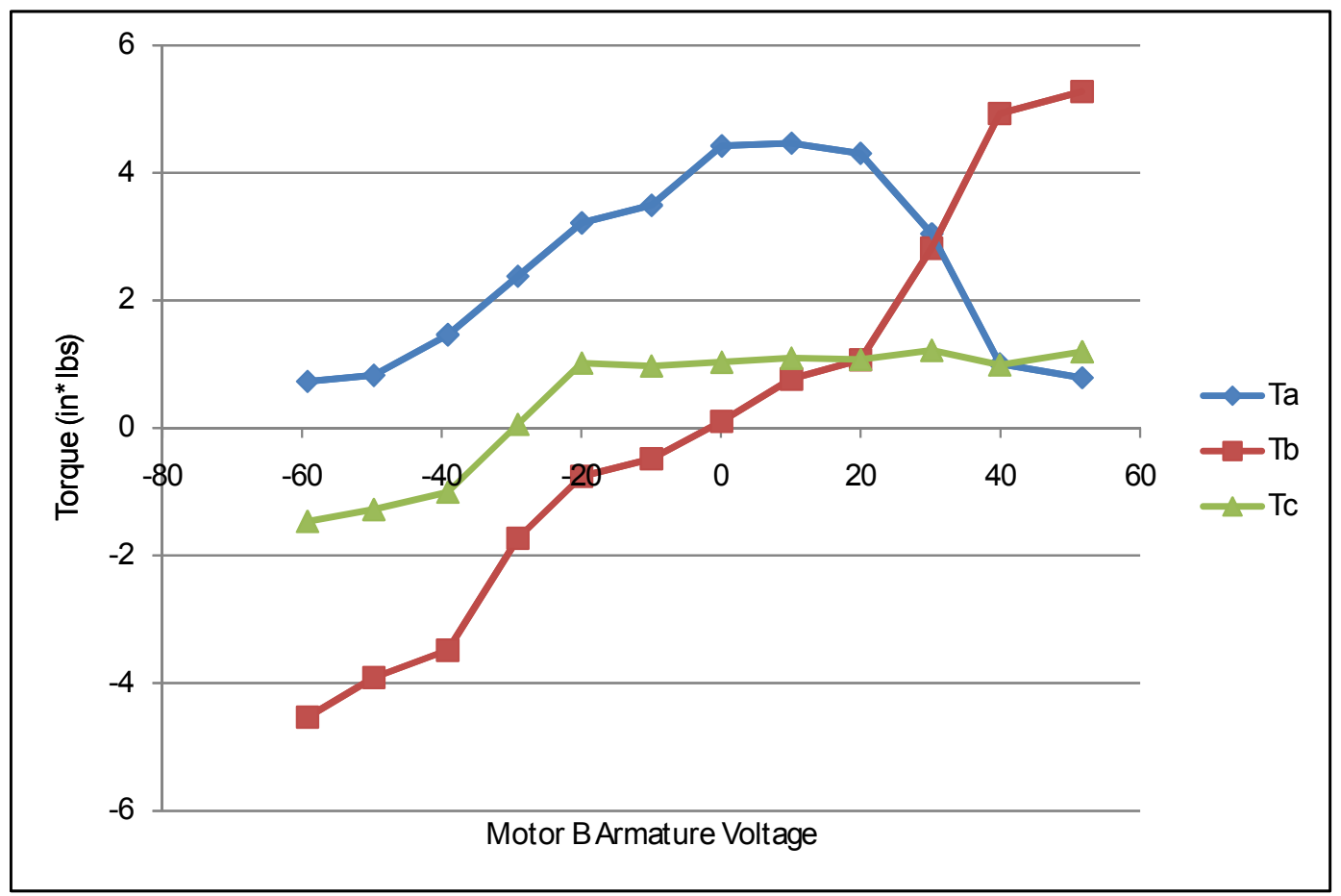

Figure 5.14: Test Cross Over Torque Output: same as Figure 4.13 
This would be useful in a hybrid vehicle if Inputs A and B did not start from zero angular velocity, as in the previous 2o test. In the cross over test it is also evident that Input B provided the majority of the torque to the output when Output $\mathrm{C}$ was rotating in the negative direction. This is not ideal as it would be preferred to have, as discussed earlier, Input A carry the bulk of the load and only use Input B as an rpm and directional governor. Nonetheless, it is significant that the differential can function as an IVT under a load. Again, it may be possible to use gear reduction from the output shaft of Motor B to the differential Input B shaft to facilitate less load on Input B and more load on Input A.

\subsection{Minimizing Backdrive Between Inputs}

To allow Input A to provide the majority of the torque through the differential to the output and Input B to primarily dictate the angular velocity and direction of the output, a means of decreasing backdrive between the inputs must be devised. To accomplish the reduction of backdrive through the Input B shaft several methods could be considered. These methods are:

1. Incorporate a worm drive between Motor B and the Input B shaft

2. Integrate a gear reduction from Motor B and the Input B shaft

\subsubsection{Incorporate A Worm Drive}

Using a worm drive on the Motor B output shaft to turn a worm gear on the differential Input B shaft would surely decrease any backdrive initiated from Input A as Output C is more heavily loaded. Figure 5.15 displays how this might be accomplished.

A worm drive is extremely effective at not being backdriven when a reverse load is applied

to it. However, a worm drive is also extremely inefficient at low rpm values when compared to straight bevel and spur gears because of the substantially increased friction between the worm drive and the worm gear. For automotive transmission applications efficiency is of primary interest. 


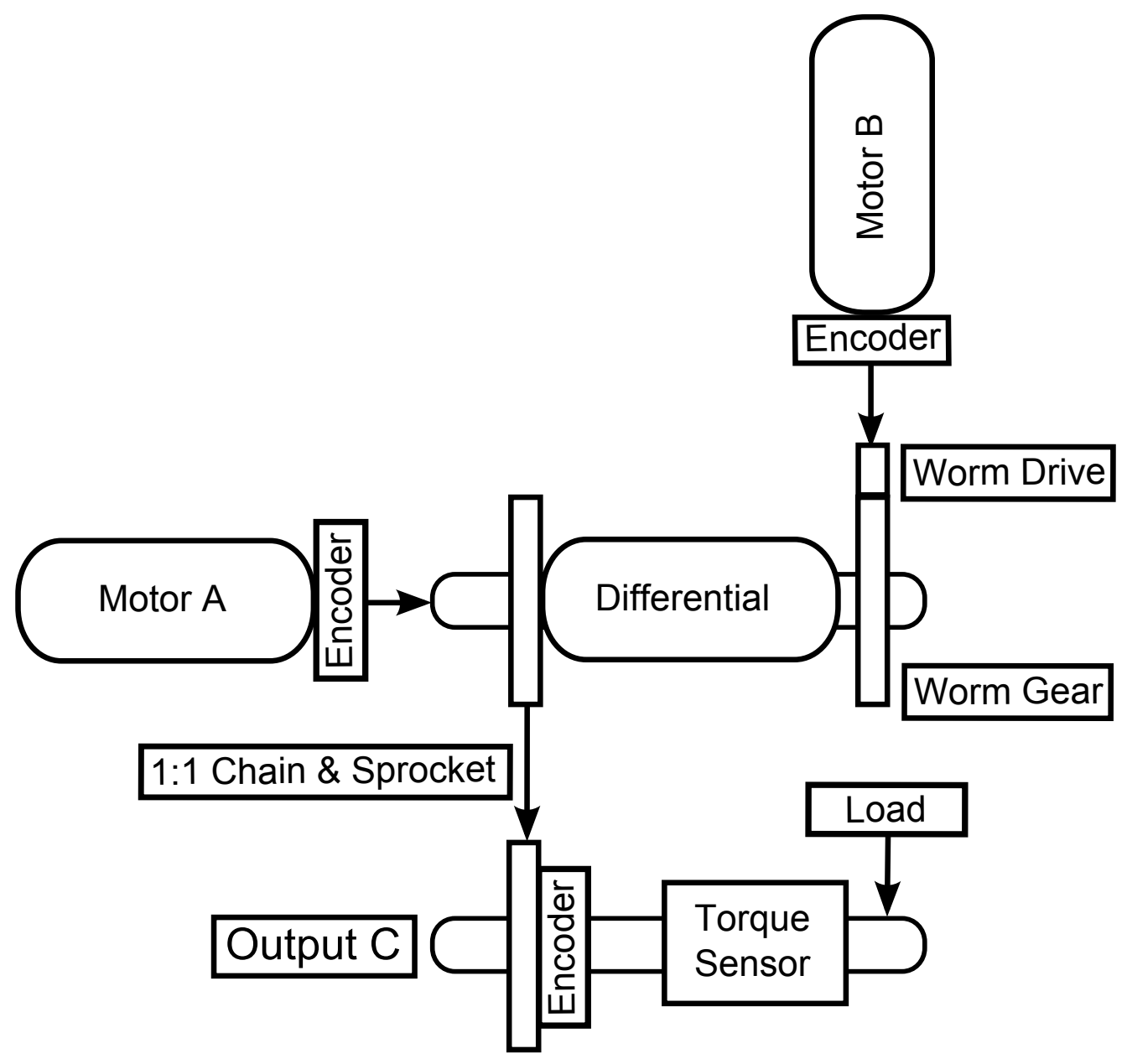

Figure 5.15: Minimizing Backdrive - Worm Drive

Compromise between a worm drive and bevel gear yields a helical or hypoid gear. Helical or hypoid gears have less friction than worm gears, in general, but offer increased resistance to backdrive when compared to bevel and spur gears.

\subsubsection{Integrate Spur Gear Reduction}

An alternative to a worm drive that also accomplishes minimum backdrive is to use spur gears in the form of a gear reduction. Although not as effective at preventing backdrive as the worm drive, spur gears are commonly used in automotive transmissions and with ratio reduction can produce good rpm/torque tradeoffs. Figures 5.16 and 5.17 display comparisons between the several gears discussed. Figure 5.18 shows gear reduction from Motor B to Input B. 


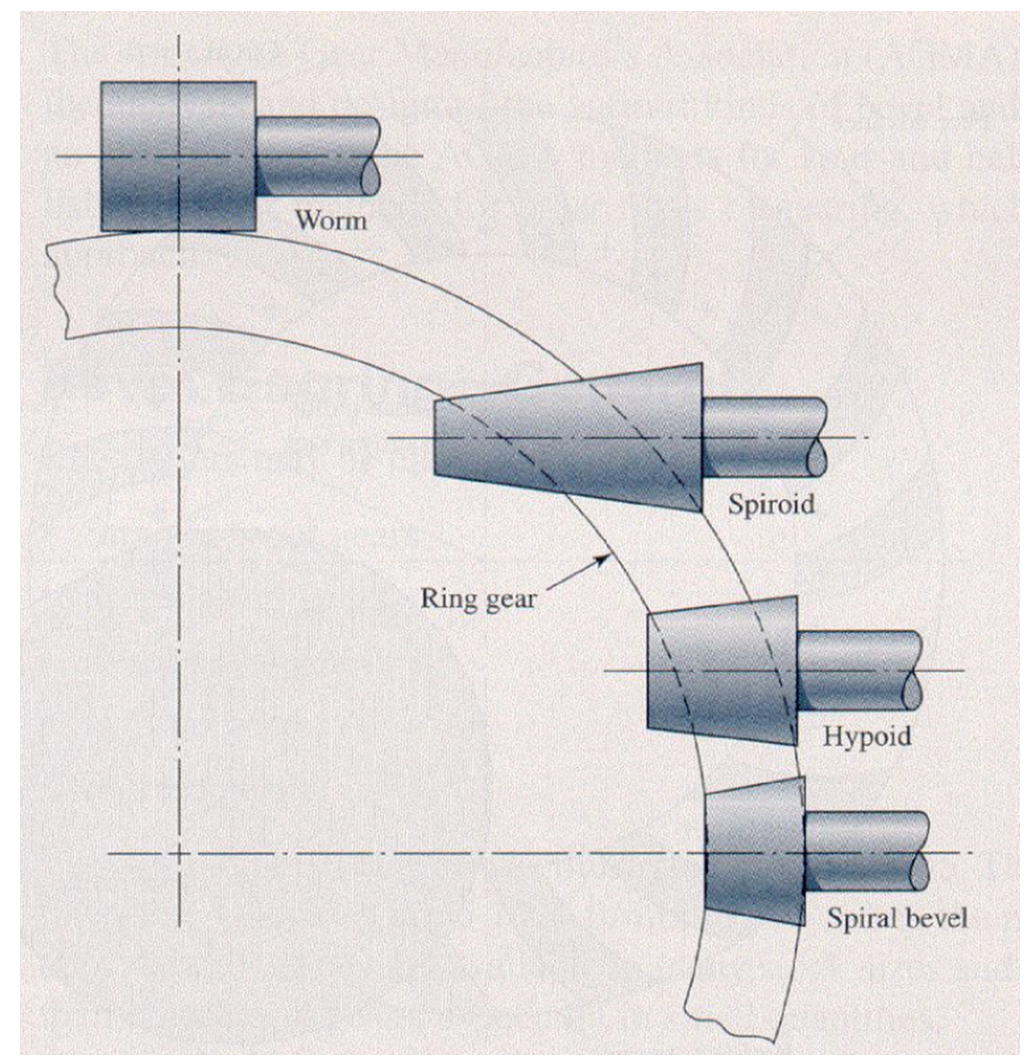

Figure 5.16: Gear Comparison [27]

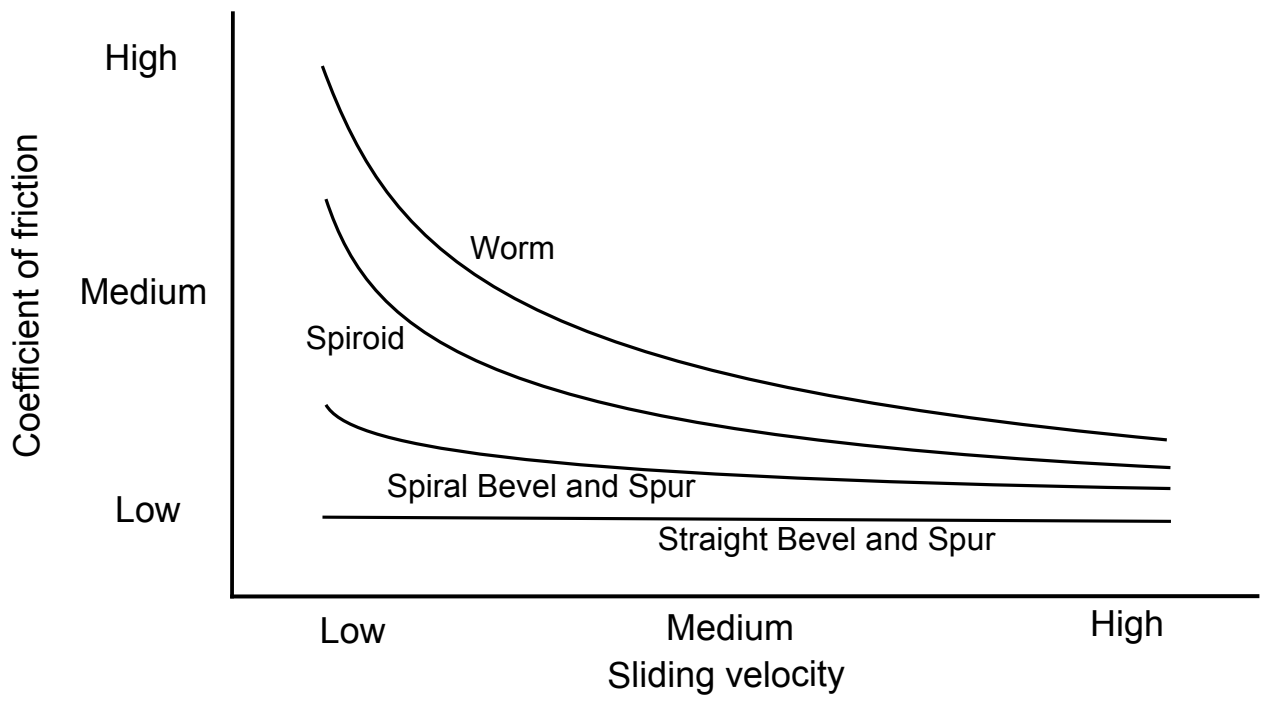

Figure 5.17: Gear Efficiency Comparison 


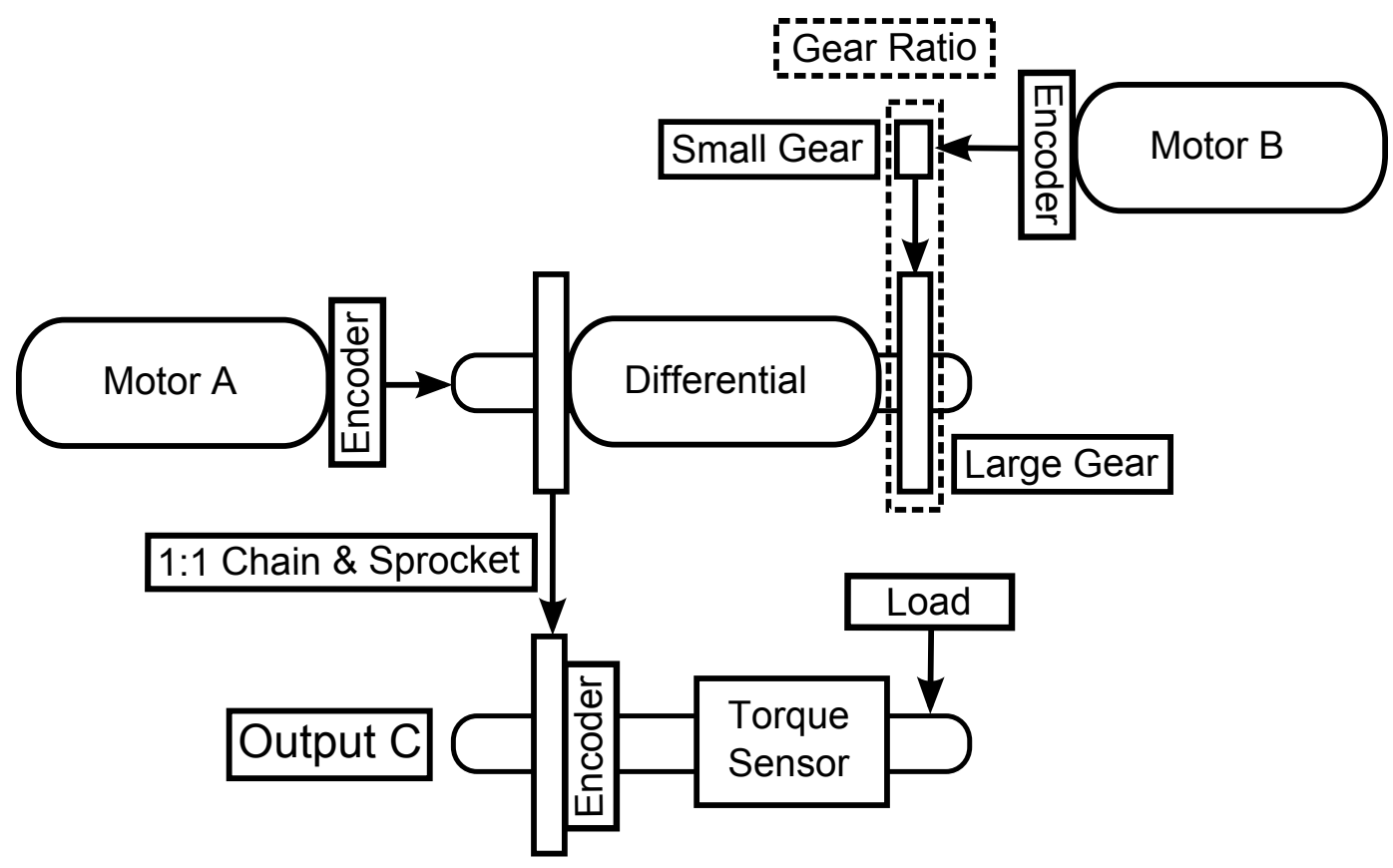

Figure 5.18: Increasing Torque Flow to Output C

To visualize how a gear reduction from Motor B to Input B would affect the torque flow in the differential refer to Figures 5.19 - 5.20.

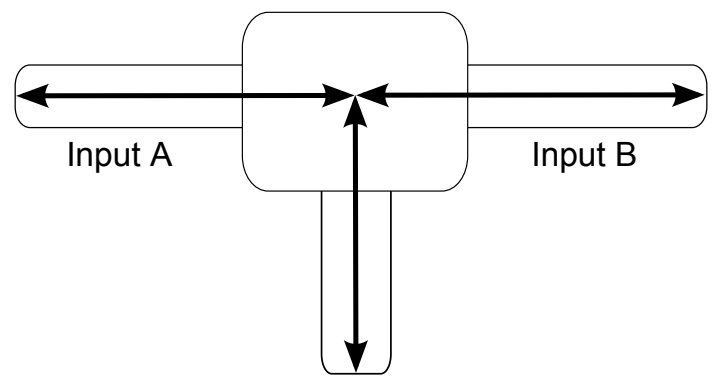

Output C

Figure 5.19: Torque Flow of a Dual-input Backdrivable Differential: same as Figure 5.7

Recall that Figure 5.19 displays how a differential without gear reduction of any kind results in three I/Os. In an effort to approach a non-backdrivable differential, as in Figure 5.20, gear reduction must be implemented. 


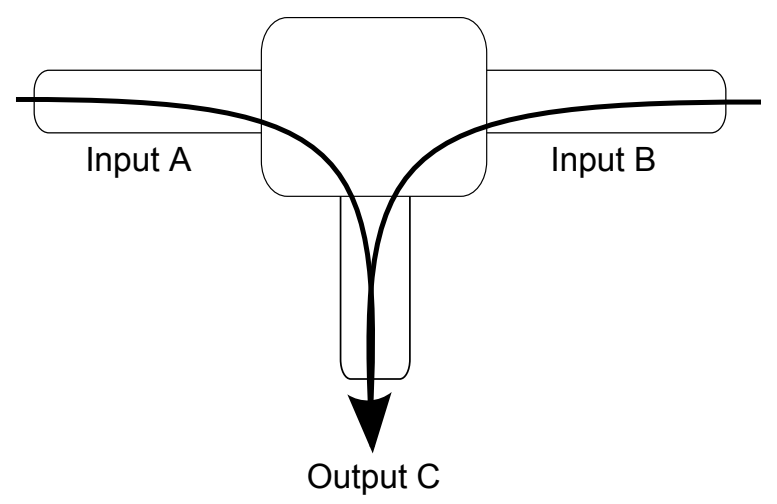

Figure 5.20: Torque Flow of a Dual-input Non-backdrivable Differential: same as Figure 3.5

Figure 5.21 depicts visually how the torque flow changes with the implementation of the gear reduction between Motor B and the differential Input B.

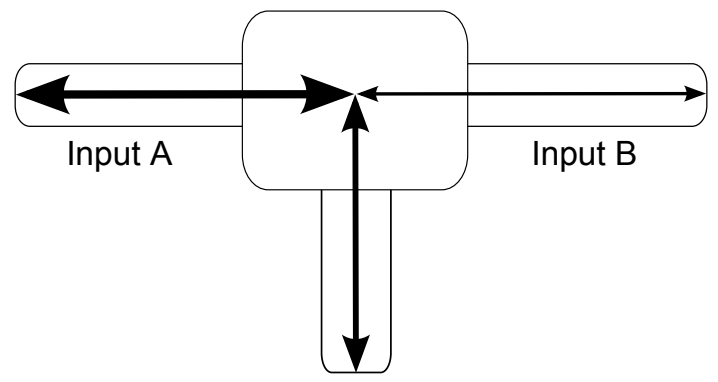

Output C

Figure 5.21: Minimizing Backdrive In A Differential

In Figure 5.21 the larger line on Input A depicts a large torque being supplied to the shaft. The lighter line on Input B shows that less torque from Input A will be likely to flow in that direction because equal torque will be entering the Input B shaft via gear reduction. The mediumsized line toward Output $\mathrm{C}$ indicates that the majority of the torque from Input $\mathrm{A}$ will flow to Output $\mathrm{C}$ because the gear reduction on Input $\mathrm{B}$ has increased its resistance to torque flow in that direction. With the gear reduction on Input B, Input A can supply more torque to a higher load at Output C. It is important to note that because of the gear reduction from Motor B to Input B, less torque is required from Motor B. However, because of this gear reduction the rpm operating 
range of Motor B must be increased to provide the same rpm range at Input B that it had previous to the implementation of the gear reduction. Relationships between rpm and torque with the gear reduction are explored hereafter.

\subsection{Equations For Gear Reduction}

The theoretical non-backdrivable differential governing equation, Equation (3.4), is restated here for reference as Equation (5.1). Recall that in this equation the inputs and outputs had no gear reductions and thus had ratios of $1: 1$.

$$
T c=-\frac{2(T a \cdot r p m A+T b \cdot r p m B)}{r p m A+r p m B}
$$

Now that there is a gear reduction from Motor B to the differential shaft Input B, the appropriate adjustments to Equation (5.1) will be made. The shaft Input B rpm, rpmB, is now no longer coupled directly to Motor B. Equation (5.2) shows the suitable modifications.

$$
\operatorname{rpmB}=\operatorname{rpmMotor} B \cdot \frac{N_{\text {motorB }}}{N_{\text {input } B}}
$$

Where rpmMotorB is the rpm of Motor B, $N_{\text {motorB }}$ is the number of teeth on the gear attached to Motor B, and $N_{\text {input }}$ is the number of teeth on the gear fastened to the Input B shaft. Equation (5.3) displays the adjustments needed for the torque portion of Equation (5.1).

$$
T b=\text { torqueMotor } B \cdot \frac{N_{\text {input } B}}{N_{\text {motor } B}}
$$

Where torqueMotorB is the torque necessary for Motor B with $N_{\text {motorB }}$ and $N_{\text {inputB }}$ as previously stated. It is important to note here that if the reduction gear set is meshed and not chain driven that $T b$ and $r p m B$ assume negative values. For a chain and sprocket gear reduction the values are positive, as shown in the preceding equations.

Substituting Equation (5.2) and Equation (5.3) into Equation (5.1) we get Equation (5.4).

$$
T c=-\frac{2\left[\text { Ta } \cdot \operatorname{rpm} A+\left(\text { torqueMotor } B \cdot \frac{N_{\text {input } B}}{N_{\text {motor } B}}\right) \cdot\left(\operatorname{rpmMotor} B \cdot \frac{N_{\text {motor } B}}{N_{\text {input } B}}\right)\right]}{\operatorname{rpm} A+\operatorname{rpmMotor} B\left(\frac{N_{\text {motor } B}}{N_{\text {input } B}}\right)}
$$


Simplifying Equation (5.4) we get Equation (5.5), the governing equation for a theoretical non-backdrivable differential using gear reduction.

$$
T c=-\frac{2 \cdot N_{\text {inputB }}(\text { rpmA } \cdot \text { Ta }+ \text { rpmMotorB } \cdot \text { torqueMotorB })}{N_{\text {input } B} \cdot \operatorname{rpm} A+N_{\text {motorB }} \cdot \text { rpmMotorB }}
$$

Because differentials are backdrivable, Equation (5.5) has very little use in practical application. However, using the fundamental rpm equation stated in Chapter 3 and the results from this work, new equations can be developed that can be used for dual-input, single-output hybrid transmission optimization. Equation (3.1) is restated here as Equation (5.6) for reference.

$$
r p m C=\frac{r p m A+r p m B}{2}
$$

Although Equation (5.5) has been proved invalid by testing, Equation (5.6) remains valid. Therefore the following constraints that a dual-input, single-output differential with one-to-one gearing throughout and uses bevel or spur type gears with inputs turning in the same direction are those expressed in Equations (5.6) and (5.7).

$$
\frac{T c}{2}=T a=T b
$$

Recall that $T a$ and $T b$ are the shaft torques that the differential experiences, not necessarily the motor input torques. It should be noted that Equation (5.7) may not require $T a$ and $T b$ to be equal if a worm drive gear reduction is used instead of a spur gear reduction. Thus, it is possible that $T a$ could carry substantially more load than $T b$. Gear reduction via worm drive will not be explored further in this work, but is suggested for future research.

Equations (5.2) and (5.3) can still be applied to Motor B and similarly to Motor A in Equations (5.6) and (5.7), resulting in Equations (5.8) and (5.9).

$$
\begin{gathered}
r p m C=\frac{\operatorname{rpmA} \cdot \frac{N_{\text {motorA }}}{N_{\text {input } A}}+\operatorname{rpmB} \cdot \frac{N_{\text {motor } B}}{N_{\text {input } B}}}{2} \\
\frac{T c}{2}=\text { torqueMotorA } \cdot \frac{N_{\text {input } A}}{N_{\text {motorA }}}=\text { torqueMotorB } \cdot \frac{N_{\text {input } B}}{N_{\text {motor } B}}
\end{gathered}
$$


Using Equations (5.8) and (5.9) makes it possible to optimize the differential transmission system. Table 5.1 shows tradeoff requirements applicable when using gear reduction from Motor B to the differential Input B shaft and no reduction from Motor A to the Input A differential shaft.

Table 5.1: Gear Reduction Optimization

\begin{tabular}{|c|c|c|c|c|c|}
\hline Transmission Components & \multicolumn{5}{|c|}{ Parameters } \\
\hline Torque Requirement $T c(f t \cdot l b s)$ & 200 & 200 & 200 & 200 & 200 \\
\hline Input A Torque $T a(f t \cdot l b s)$ & 100 & 100 & 100 & 100 & 100 \\
\hline Input B Reduction Ratio & $1: 1$ & $2: 1$ & $5: 1$ & $10: 1$ & $15: 1$ \\
\hline Motor B Torque $T b(f t \cdot l b s)$ & Highest & High & Medium & Low & Lowest \\
\hline Motor B RPM $r p m B$ & Lowest & Low & Medium & High & Highest \\
\hline Back Drive Between Inputs & Highest & High & Medium & Low & Lowest \\
\hline
\end{tabular}

\subsubsection{Governing Parameters}

To solve for the necessary gear reductions in the system the governing parameter must be known. For the application in a vehicle this parameter is the maximum wheel torque and rpm requirement for vehicle performance. For a 1:1 ratio differential the following parameters can be found from the torque and rpm requirement:

1. Input $\mathrm{A}$ torque $(\mathrm{Ta})$ is half of the required torque using a spur gear reduction and up to the required torque $(T c)$ if Input B uses a worm drive reduction

2. Input A rpm (rpmA) should be where the ICE is most efficient (least fuel consumption)

3. Motor B rpm (rpmMotorB) must have as large a maximum rpm range as possible to facilitate overall maximum range through the differential

4. Motor B torque (torqueMotorB) is according to the torque performance curve for the motor selected so that through the reduction ratio the torque to Input B must be half the required torque with spur gear reduction, or what is required for a worm drive reduction 
5. The necessary gear reduction can be deduced to satisfy the equal torque sharing of Input A and B with spur gear reduction. The reduction needed can also be applied to worm drive reduction.

It is important to note that the torque requirements from Motor B will change depending on the design of the gears used in the gear reduction. In addition to the gear design one should carefully consider how the torque and rpm curves interact for Motor B to assure that the torque requirements will be met as the motor varies in rpm value.

\subsection{Planetary Differential}

In conjunction with gear reduction a better differential system is necessary to facilitate optimization of the differential transmission. Making sure torque and efficiency capabilities are maintained or improved is a top priority when dealing with vehicle propulsion. To maintain a dual-input, single-output transmission system while meeting torque and efficiency requirements, a planetary differential is suggested. The differentials outlined previously used bevel or helical gears. While the gains in switching from these types of gears to spur gears may be negligible, the change in arrangement may provide significant advantages. Understanding concepts that govern the analysis of such systems is helpful in understanding advantages to the recommended arrangement. For details regarding the analysis of such systems refer to 'The Lever Analogy: A New Tool in Transmission Analysis' [28].

Figures 5.22 and 5.23 display the proposed planetary differential. As can be seen in the figures there is no ring gear. Instead there are two sun gears, the red and the blue gears. The yellow and orange gears are meshing planet gears, while the red sun gear meshes only with the orange planet gears and the blue sun gear meshes only with the yellow planet gears. The planet carrier is the grey housing and the black shaft the system output shaft. The inputs to this system would be on the red and blue sun gears. 


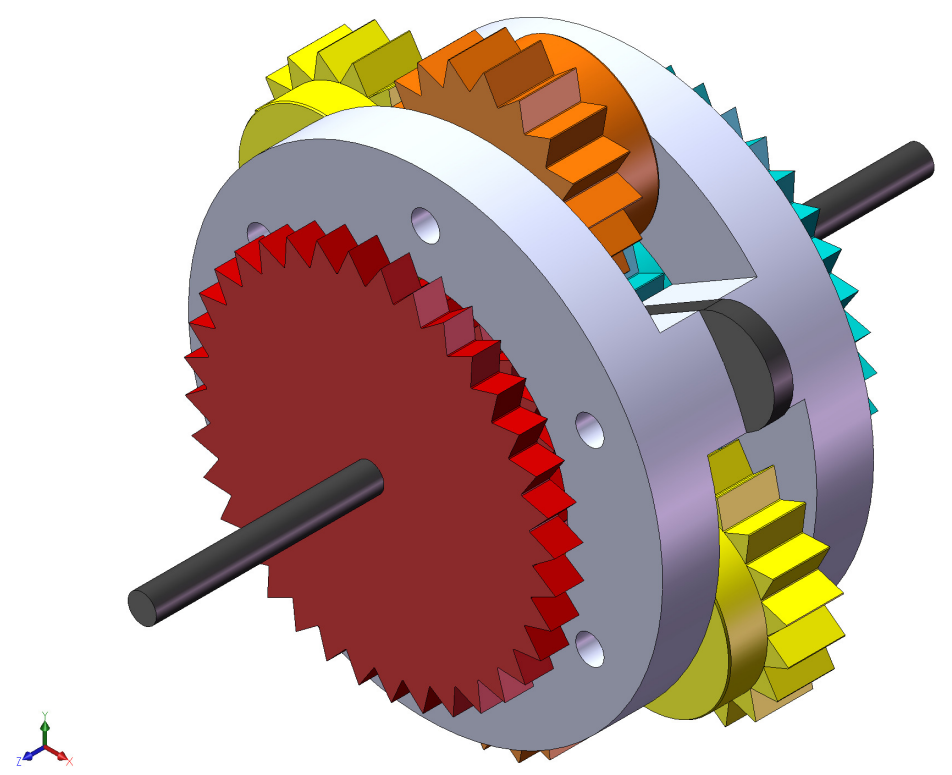

Figure 5.22: Planetary Differential - Isometric View

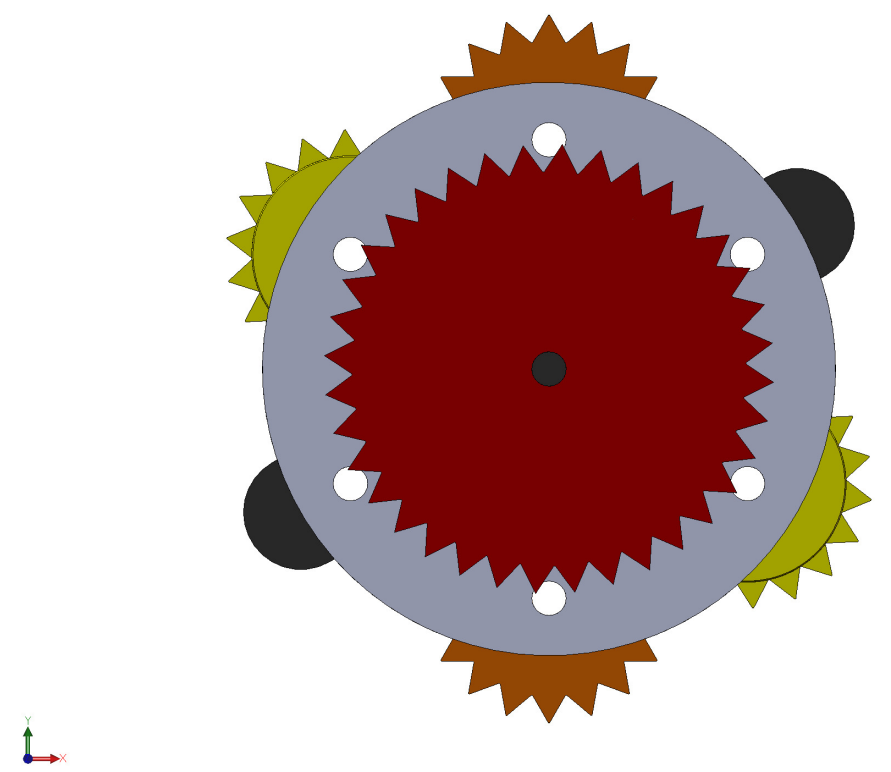

Figure 5.23: Planetary Differential - Front View 
Figures 5.24 and 5.25 show views of the mesh between sun and planet gears. The grey planet carrier and black output shaft have been excluded from these figures to aid in clearly viewing internal gear mesh. Note again that the red sun gear meshes only with the orange planet gears and the blue sun gear meshes only with the yellow planet gears.

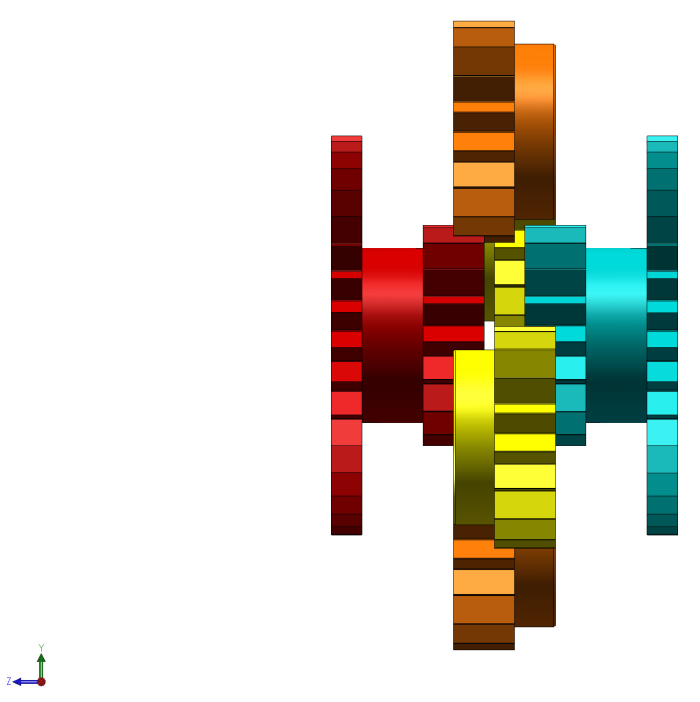

Figure 5.24: Planetary Differential - Side View

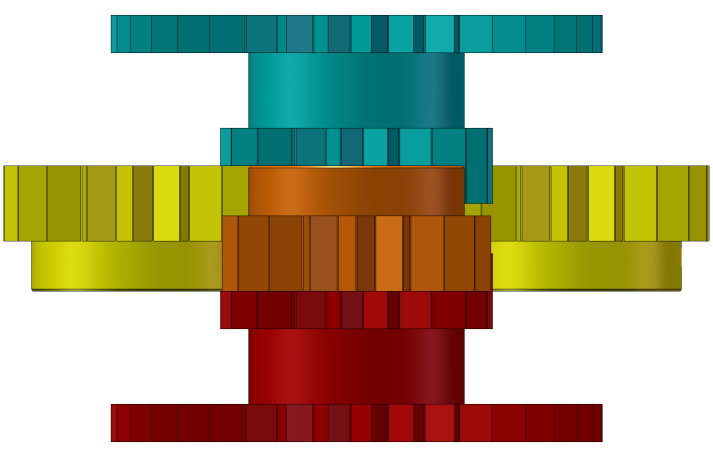

$\Gamma$

Figure 5.25: Planetary Differential - Top View 
A visual representation of the new basic layout of the system, including Motor A, Motor B, and the planetary differential, can be seen in Figure 5.26.

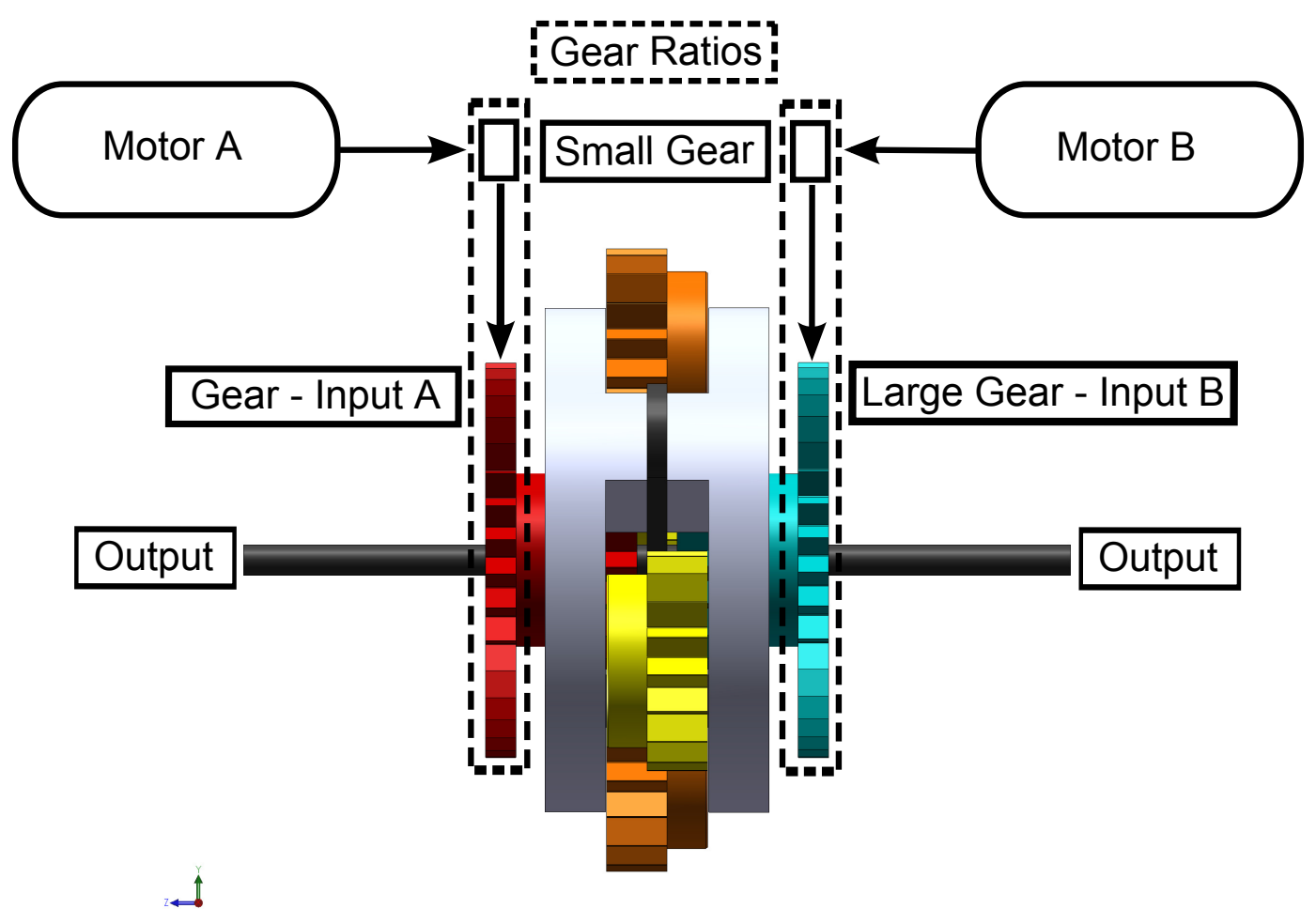

Figure 5.26: Planetary Differential - Power Transmission

\subsection{Planetary Differential Advantages}

There are several advantages of using a planetary differential over using a conventional differential or planetary gear set (PGS). By using a planetary differential the design space of the gear ratios within and connecting to the differential is enlarged. Specifically, not having a ring gear to constrain the design space of the gear pitch diameters may be highly advantageous. Further explanation on the advantages of a planetary differential are explored hereafter. 


\subsubsection{Advantages Over A Conventional Differential}

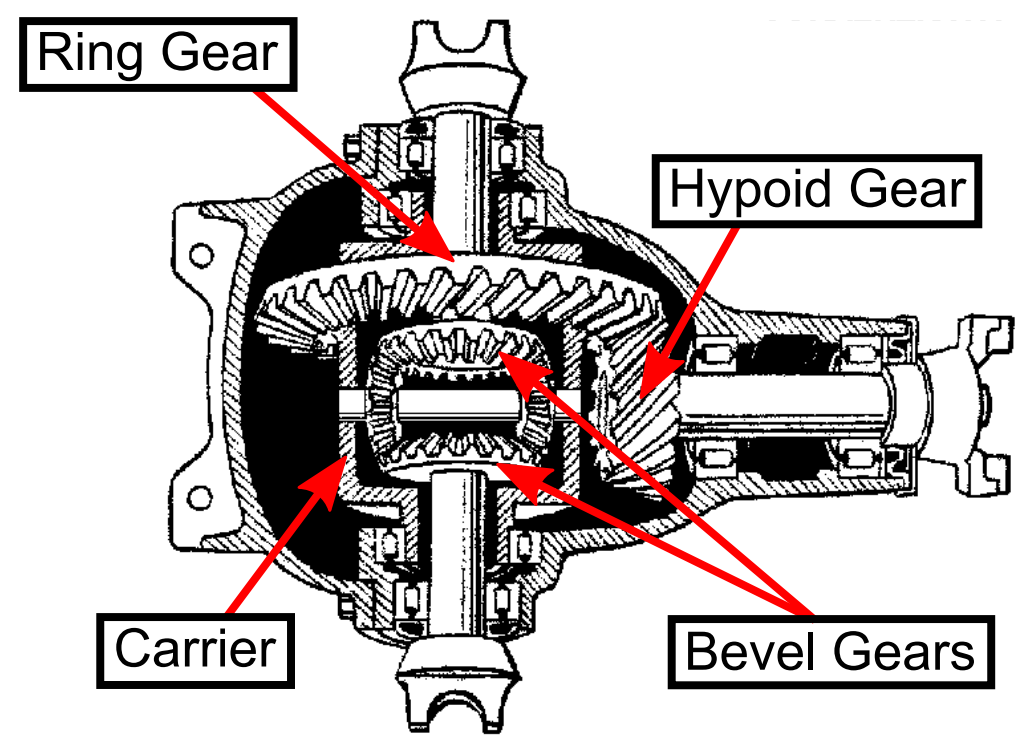

Figure 5.27: Conventional Differential

In the conventional differential the ring gear encircles the carrier and the carrier encompasses the bevel gears. See Figure 5.27. This means that the carrier can be no larger than the inside diameter of the ring gear, constraining the maximum diameter of the bevel gears. In addition to the bevel gear diameter constraint, the ratio between the two bevel gears can be nothing other than $1: 1$, which is not a problem when using the differential in the conventional way with one input and two outputs. However, when used as a dual-input, single-output device, as in this work, it is desirable that the ratios within the differential be optimized. The optimization of the gear ratios, to facilitate Motor B having a lower torque requirement than Motor A, necessitates variation in gear ratios between the bevel gears. A planetary differential has no ring gear and therefore allows gear sizes to have a wider diameter range. Also, because the planetary differential does not use bevel gears the ratios can be changed.

In addition to the increase in gear ratio design space the use of a hypoid gear is no longer needed. As discussed earlier, the inefficiencies of a worm drive limit its use in powertrain applications. A hypoid gear is a hybrid of a straight bevel gear and a worm drive; therefore some of the 
inefficiencies inherent in worm drives reside in hypoid gears. Because a planetary differential does not use worm drives or hypoid gears it has the potential to be more efficient.

\subsubsection{Advantages Over A Planetary Gear Set}

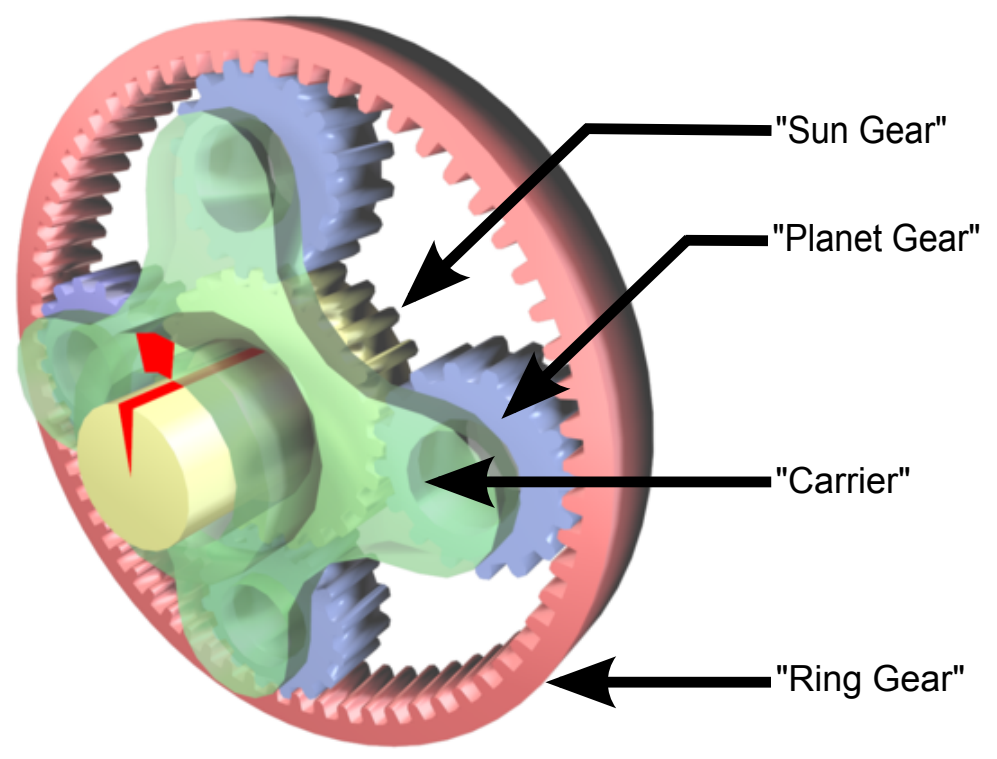

Figure 5.28: Planetary Gear Set

Similar to the conventional differential, the ring gear in a PGS constrains the optimization of the gear ratios. In a PGS one of the two gear mesh ratios is chosen and the other is set as a function of the first. For example, in Figure 5.28 one might choose the number of teeth needed on the ring and planet gears for a certain ratio and as a result, the sun gear must be a certain diameter to make positive engagement to the planets possible. In this way the ring gear in a PGS limits the optimization of ratios for the device.

Figure 5.29 shows that because of the way the gears mesh in a planetary differential, a ratio nearer the optimum system requirement may be chosen. It can be observed in the figure that three gears (two ratios) can be changed and still allow the fourth to have a range in which it can also be altered. For example, the red sun gear could have a certain ratio with the orange planets, the orange planets a different ratio with the yellow planets, and the yellow planets a still different ratio 
with the blue sun gear. Moving from a conventional differential or PGS to a planetary differential allows a broader range of gear ratio optimization and thus holds promise for future hybrid vehicle powertrain applications.

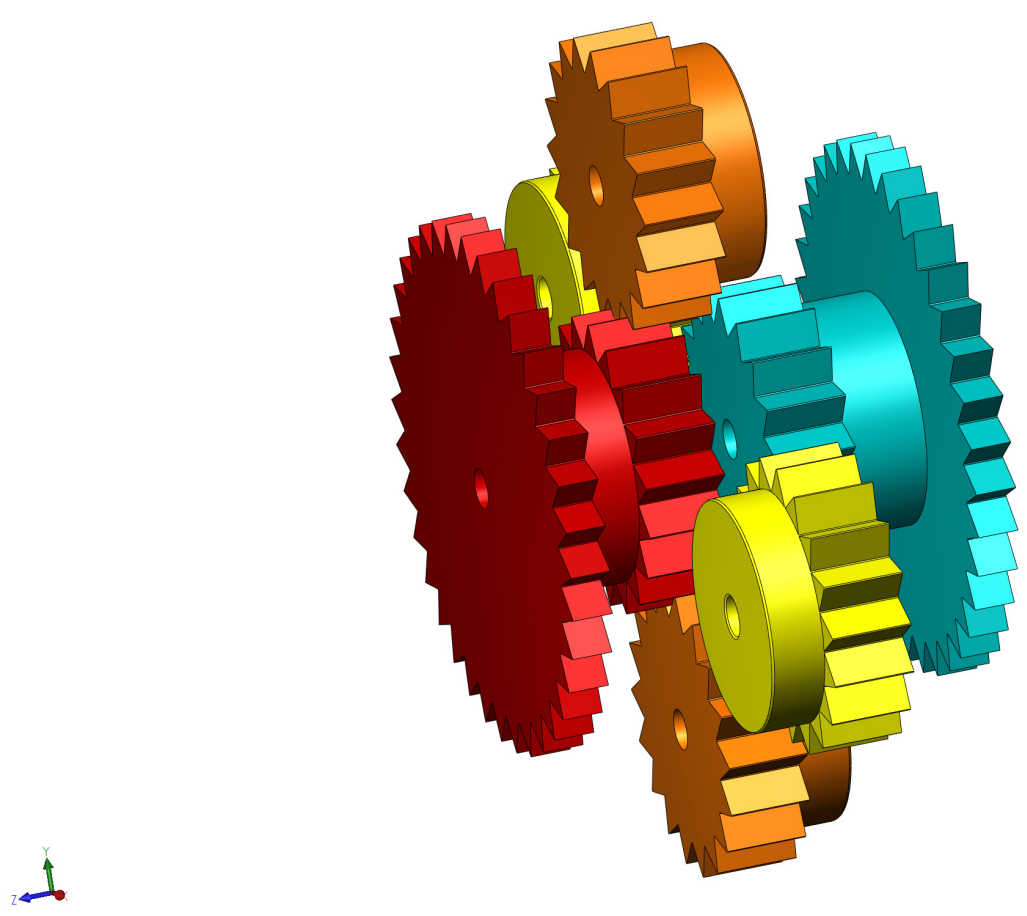

Figure 5.29: Planetary Differential - Ratio Optimization

\subsubsection{Practical Constraints Of A Planetary Differential}

Although not investigated in this work, there are practical design constraints for the use of a planetary differential in a powetrain application. Several of the practical constraints that should be considered in the optimization of a planetary differential are:

1. The limits to which gear diameters can extend without interfering with non-meshing gears

2. The vibration effects gear sizes have on the transmission device 


\subsubsection{Mechanical Differential Comparison}

Figure 5.30 displays the several mechanical differentials discussed in this work. Table 5.2 shows how these mechanical differentials compare in several areas pertinent for use in power combination devices. It should be noted that this comparison takes into account efficiency due to gear arrangement and not necessarily gear type, as gear type may have differing implications pertaining to each mechanical differential.

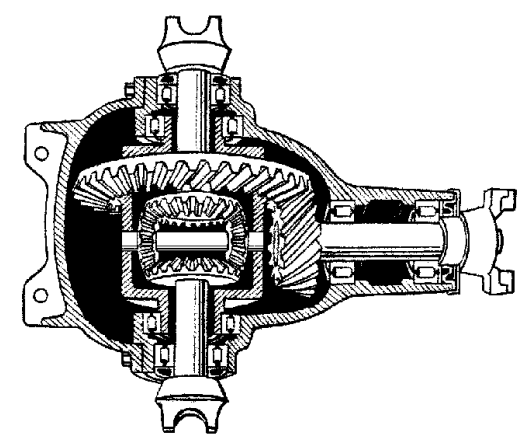

Common Differential

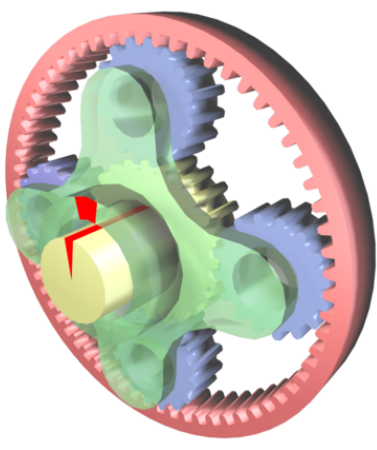

PGS

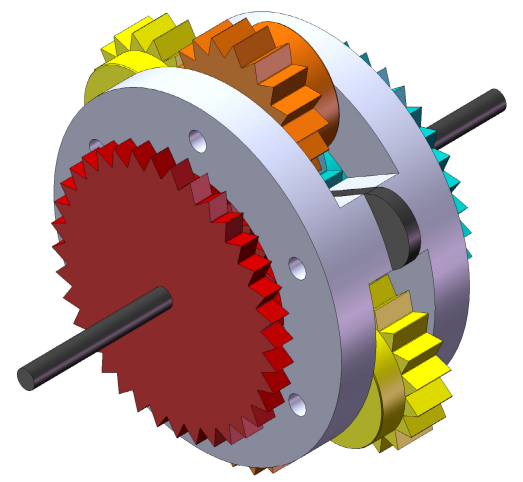

Planetary Differential

Figure 5.30: Mechanical Differential Comparison

Table 5.2: Mechanical Differential Comparison

\begin{tabular}{|c|c|c|c|}
\hline Parameter & Differential & PGS & Planetary Differential \\
\hline Compactness & Low & High & Medium \\
\hline Optimization & Low & Medium & High \\
\hline Efficiency & Medium & High & High \\
\hline Load Capacity & High & High & High \\
\hline Availability & High & Medium & Low \\
\hline
\end{tabular}

Through the comparison in Table 5.2 we can see that a planetary differential may provide very similar characteristics as a planetary gear set in terms of load capability, efficiency, and compactness. The planetary differential's backrive, like any mechanical differential, will depend on 
the gear reduction and gear type. However, as discussed previously it may also have increased design and optimization space to enable larger gear variance in size and type than other mechanical differentials.

\subsection{Example}

Whether or not one decides to use a planetary differential, it is important to understand the implications of being able to use a differential as an IVT. For this reason, the following example will explore the inferences of using a differential as a dual-input, single-output device, with one input at a relatively constant angular velocity and the other input as the speed control with varying angular velocity. The example is based on an outside wheel diameter of 24 inches.

\subsubsection{RPM Implications}

This example will explore the implications visually using a nomograph. The following nomographs have three vertical axes, each representing one of the rotational inputs or the rotational output. Figure 5.31 gives a visual representation of the vehicle layout for this example. As can be

observed in Figure 5.31, this example has reduction ratios throughout the vehicle layout. Some of the reductions are within or appending to the differential. The remaining reduction is the final gear reduction in the traditional differential.

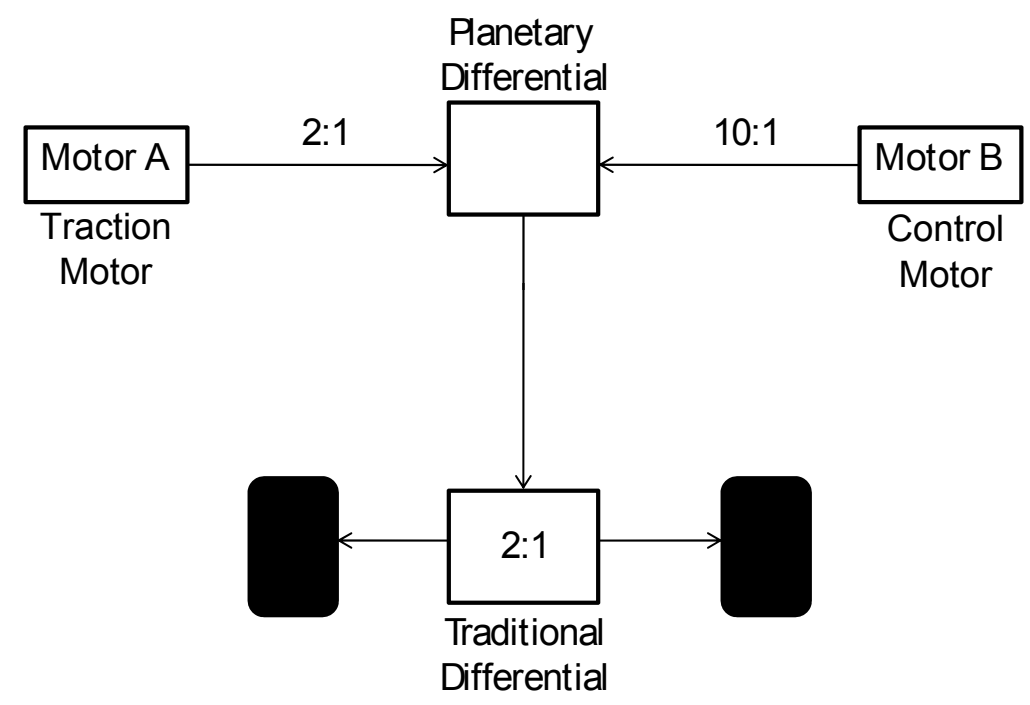

Figure 5.31: Vehicle Layout Example 
It is observed here that the reduction from Motor A is 2:1, meaning that this motor is the motor that will provide the majority of the torque for forward vehicle propulsion. Motor A will also have very little rpm variation. It can also be observed that because of the larger 10:1 reduction from Motor B that this motor will provide a wide range of rpm capability with less torque requirement. In this way the differential experiences equal torque on Input A and Input B while the sources of the respective torque (Motors A and B) will deliver largely differing torque and rpm values. More discussion on torque will follow near the end of this example. Here the primary focus will be directed toward the IVT characteristics of this setup. Figure 5.32 shows that with these system gear reductions, Motor A will reside at a constant rpm of 3,000. Motor B's rpm value, indicated by the red dot, can fluctuate anywhere along the vertical axis. As the rpm value for Motor B changes the velocity of the vehicle changes, as specified on the axis furthest to the right. Figures $5.32-5.35$ show that although the rpm value of Motor A does not change significantly the vehicle speed does change because of the considerable variance in the Motor B rpm value.

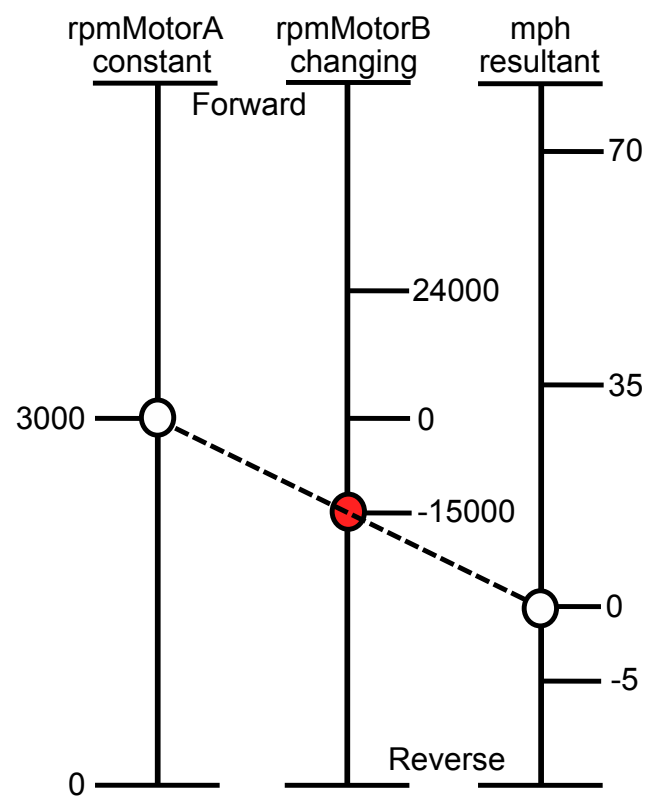

Figure 5.32: Output RPM Dependencies - Vehicle Stopped

In Figure 5.32 it is observed that the vehicle velocity is zero while the system inputs are nonzero. As stated previously in this work, one of the defining attributes of an IVT is having a zero output with non-zero inputs. 


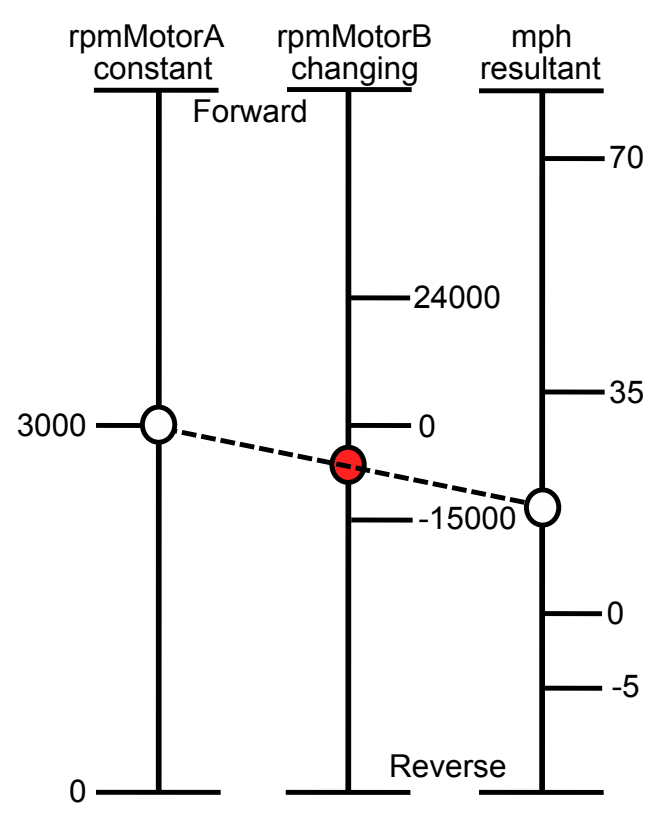

Figure 5.33: Output RPM Dependencies - Vehicle Speed Low

Figure 5.33 displays that as the rpm of Motor B moves from a large negative value toward a zero angular velocity the result is forward vehicle acceleration.

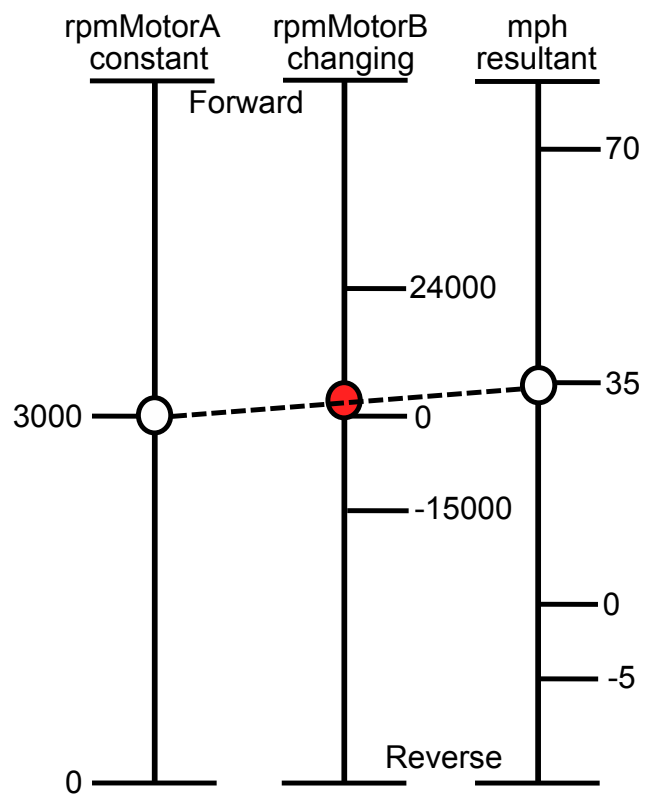

Figure 5.34: Output RPM Dependencies - Vehicle Speed Medium 
As Motor B slows to zero angular velocity, changes polarity, and begins to accelerate in the opposite direction, it is observed that an increase in vehicle speed continues to be achieved. This is shown in Figure 5.34 where the red indicator has just passed the zero angular velocity mark. Figure 5.35 displays the higher vehicle velocity resulting from a continued increase of the Motor B rpm value.

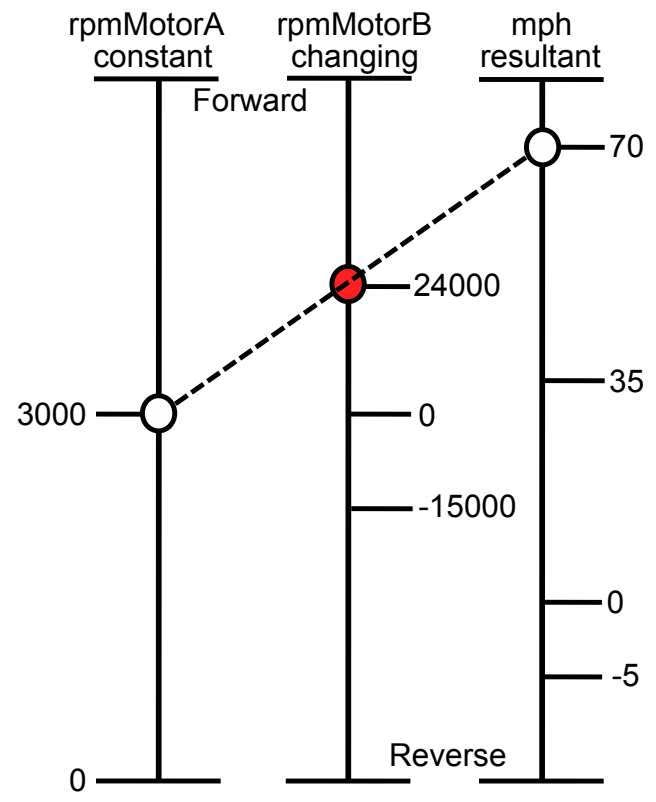

Figure 5.35: Output RPM Dependencies - Vehicle Speed High

It is important to note here that continued vehicle acceleration can only be accomplished by a Motor B polarity change. The instance of the polarity change with respect to vehicle speed can be varied by increasing or decreasing the gear ratios within the vehicle layout. A gear reduction change increases or decreases the space between the vertical rpm axes in the nomograph. For example, if the final gear reduction in the traditional differential of $2: 1$ is changed to $1: 1$ the vehicle speed increases as in Figure 5.36. The implications for torque due to this ratio change will be discussed shortly. 

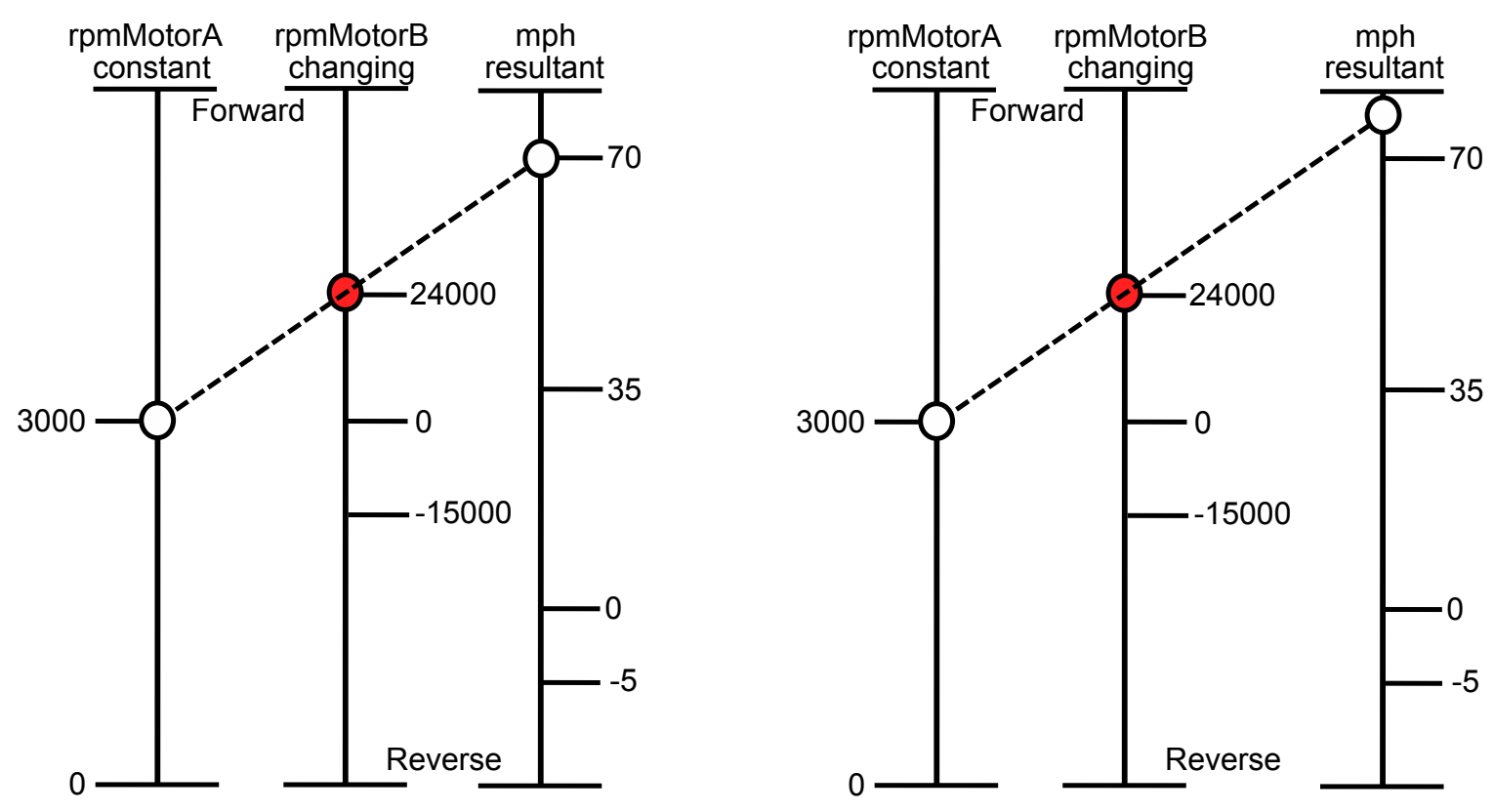

Figure 5.36: Output RPM Dependencies - Ratio Change

\subsubsection{Torque Implications}

The following nomographs indicate torque relationships for this example. Recalling that a differential is a torque split device and incorporating the given reduction ratios for this example, Figure 5.37 displays the torque relationships for this system. Figures $5.37-5.39$ show that as the torque requirement changes with vehicle acceleration, the dependant requirements for each input change accordingly.

For the following torque nomographs the torque requirement is indicated on the left vertical axis. The center vertical axis indicates the torque requirement for Motor A with the previously specified gear reduction of 2:1. Likewise, the torque requirement for Motor B is indicated on the vertical axis on the right with its reduction ratio of 10:1. The negative and positive values on the axes correspond with the negative and positive rpm values in the previous nomographs. Following the torque nomographs, a side-by-side representation of rpm and torque values will be introduced to aid in the visualization of the several instantaneous rpm and torque requirements for this example. 


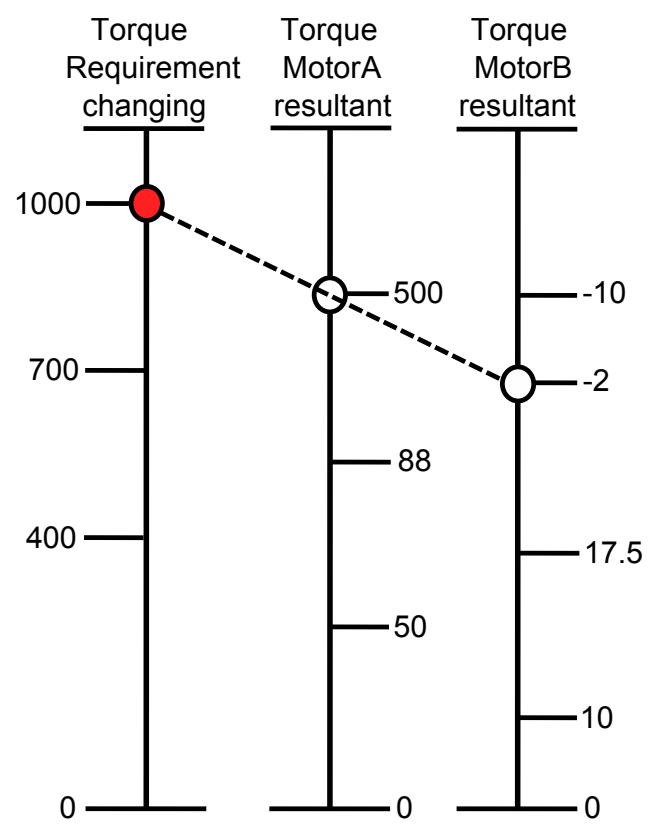

Figure 5.37: Torque Dependencies - Vehicle Accelerating From A Stop

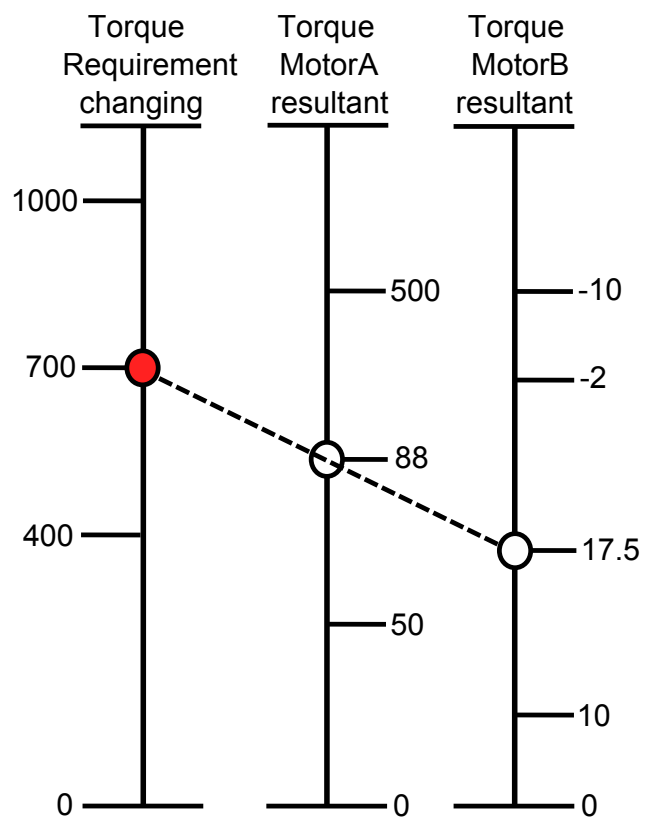

Figure 5.38: Torque Dependencies - Vehicle Accelerating

Note the large drop in torque requirement for Motor A between Figure 5.37 and Figure 5.38. This large drop in torque requirement is due to the fact that Motor B changes polarity from a direc- 
tion opposite to Motor A to an angular velocity in the same direction as Motor A. This important observation indicates that a differential is a torque split device when the inputs, Motor A and Motor $\mathrm{B}$, are both turning in the positive or negative direction. When one input is turning in the positive direction and one is turning in the negative direction, the differential is no longer a torque split device. This is because of the discussion earlier on the backdrivability of the differential. It is evident that gear reduction helps in this facet, but does not entirely solve the problem of backdrive. This means that only the input turning in the same direction as the output supplies torque to the output. Also, if the load on the output is large enough, backdrive will still occur. However, integrating a physical brake on the Motor B shaft is one way to overcome the problem of backdrive between Motor A and Motor B. A brake can aid in the deceleration of Motor B and only allow Motor A's torque to be directed to the output. The result is that Motor A must carry the entire load and thus the spike in the torque requirement for Motor A on the nomograph. The same would be true of Motor B if the desired vehicle direction is reverse.

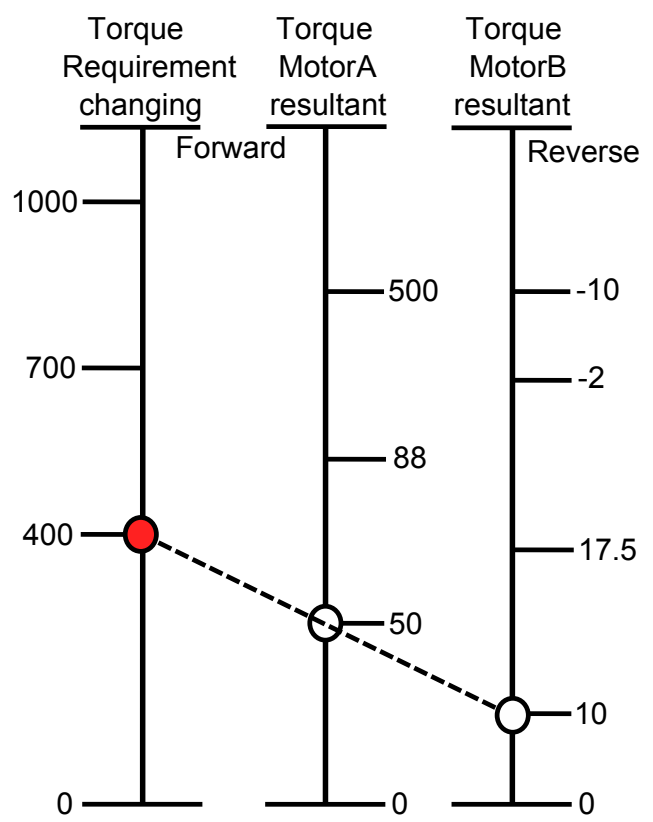

Figure 5.39: Torque Dependencies - Vehicle With High Velocity And Minimal Acceleration

Figure 5.39 shows the continuation of the decreasing torque requirement at higher wheel rpm values. Another attribute that Figures 5.37 - 5.39 show is that the torque line translates ver- 
tically, but does not rotate as did the rpm nomograph. The fact that the torque line translates and does not rotate is because of the set gear reduction throughout the vehicle layout.

The final gear reduction change discussed earlier showed that the vertical axis representing vehicle velocity moved to the right and increased vehicle speed. Figure 5.40 displays the effect the same reduction change $(2: 1$ to $1: 1)$ has on torque.
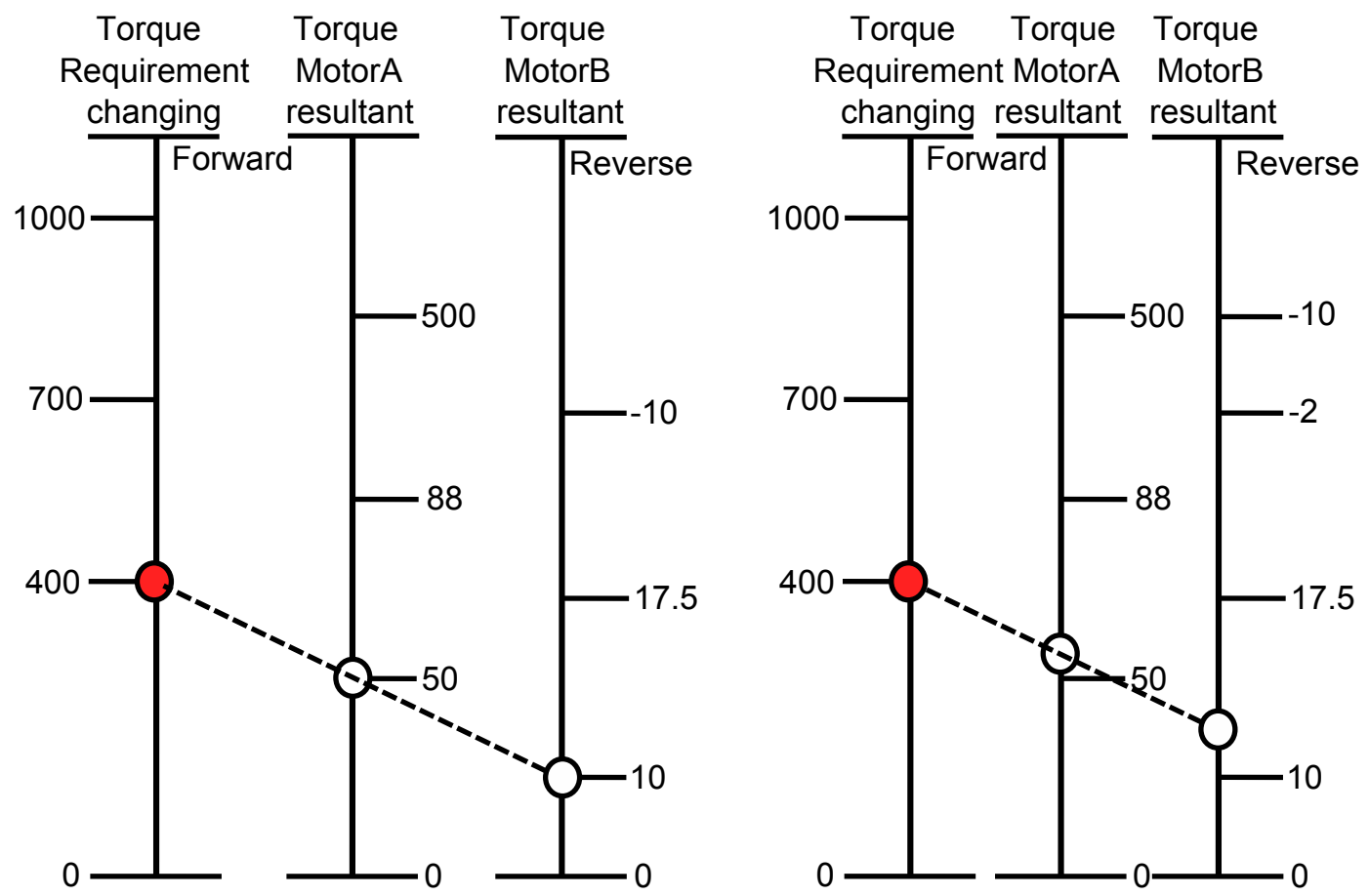

Figure 5.40: Torque Dependencies - Vehicle With High Velocity And Minimal Acceleration

Notice here that instead of moving one vertical axis both of the dependent axes moved closer together. The respective torque requirements on each input increased without the load on the output increasing. As is always the case, an increase in rpm due to a physical gear size change requires more torque to be supplied from the input(s).

\subsubsection{Visualization}

This section places the rpm and torque nomographs side by side to aid in visualizing the simultaneous performance requirements. Figures 5.41 - 5.45 display the respective scenarios. 

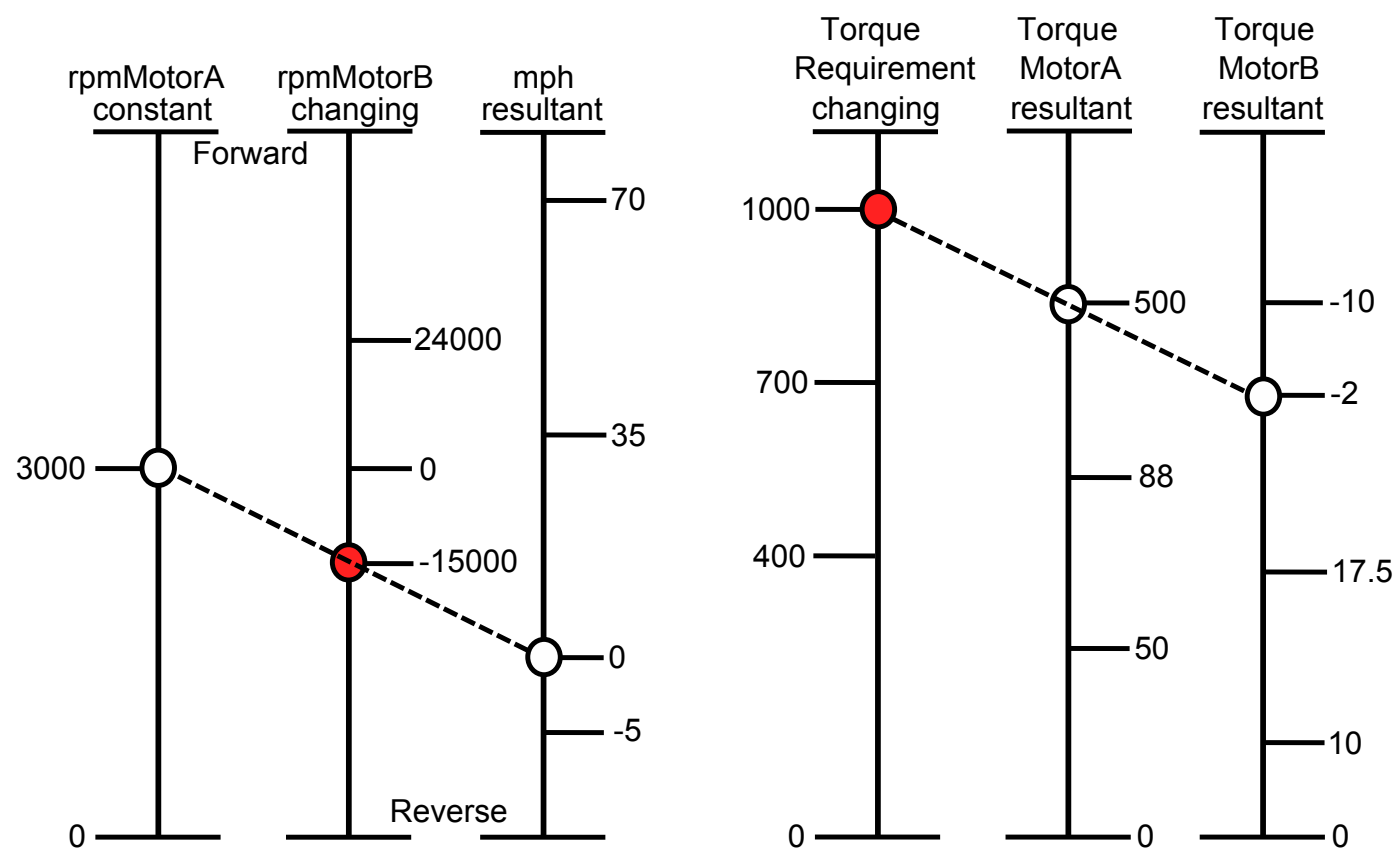

Figure 5.41: Visualization - Vehicle Accelerating From A Stop
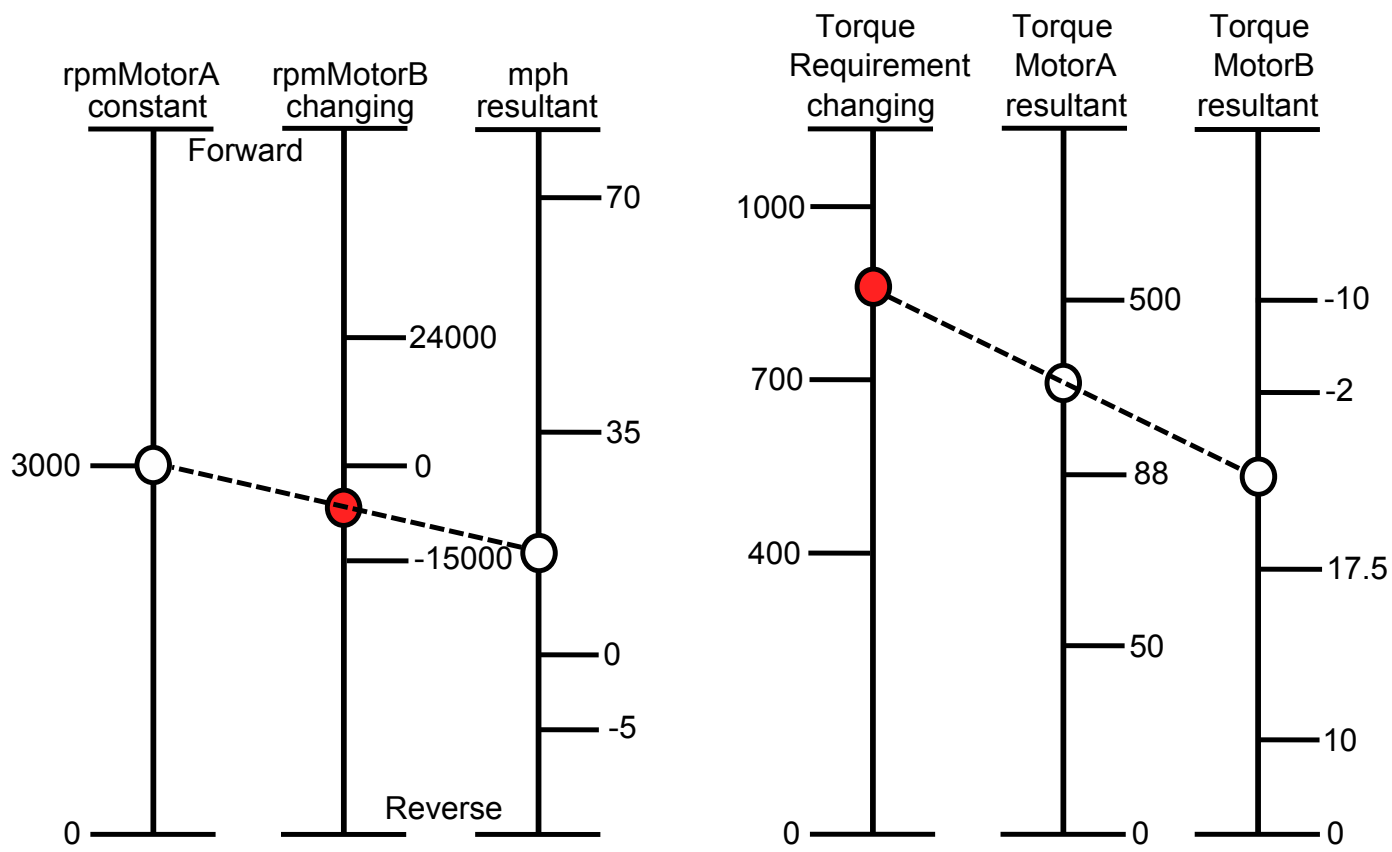

Figure 5.42: Visualization - Vehicle Accelerating 1 


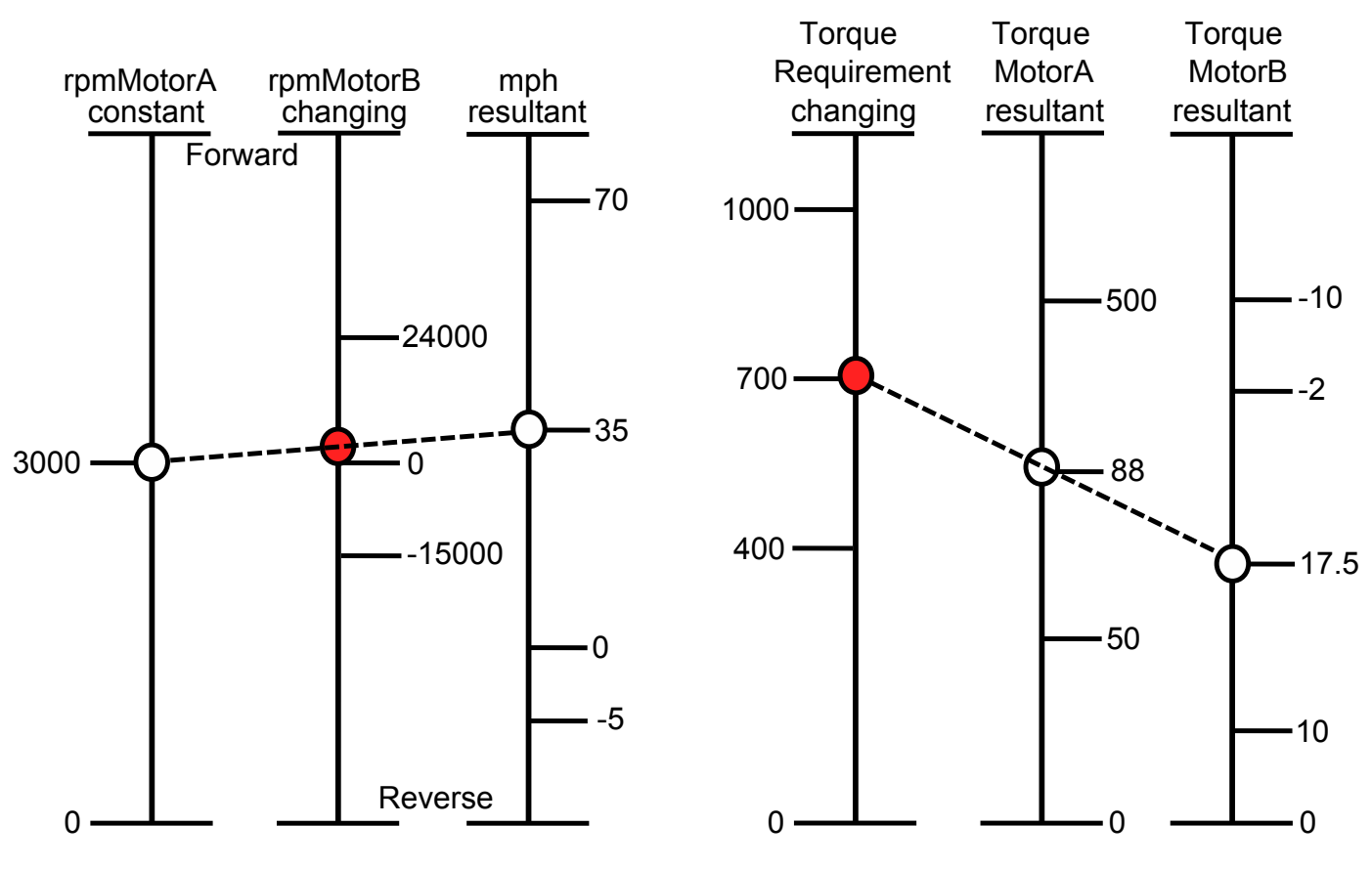

Figure 5.43: Visualization - Vehicle Accelerating 2
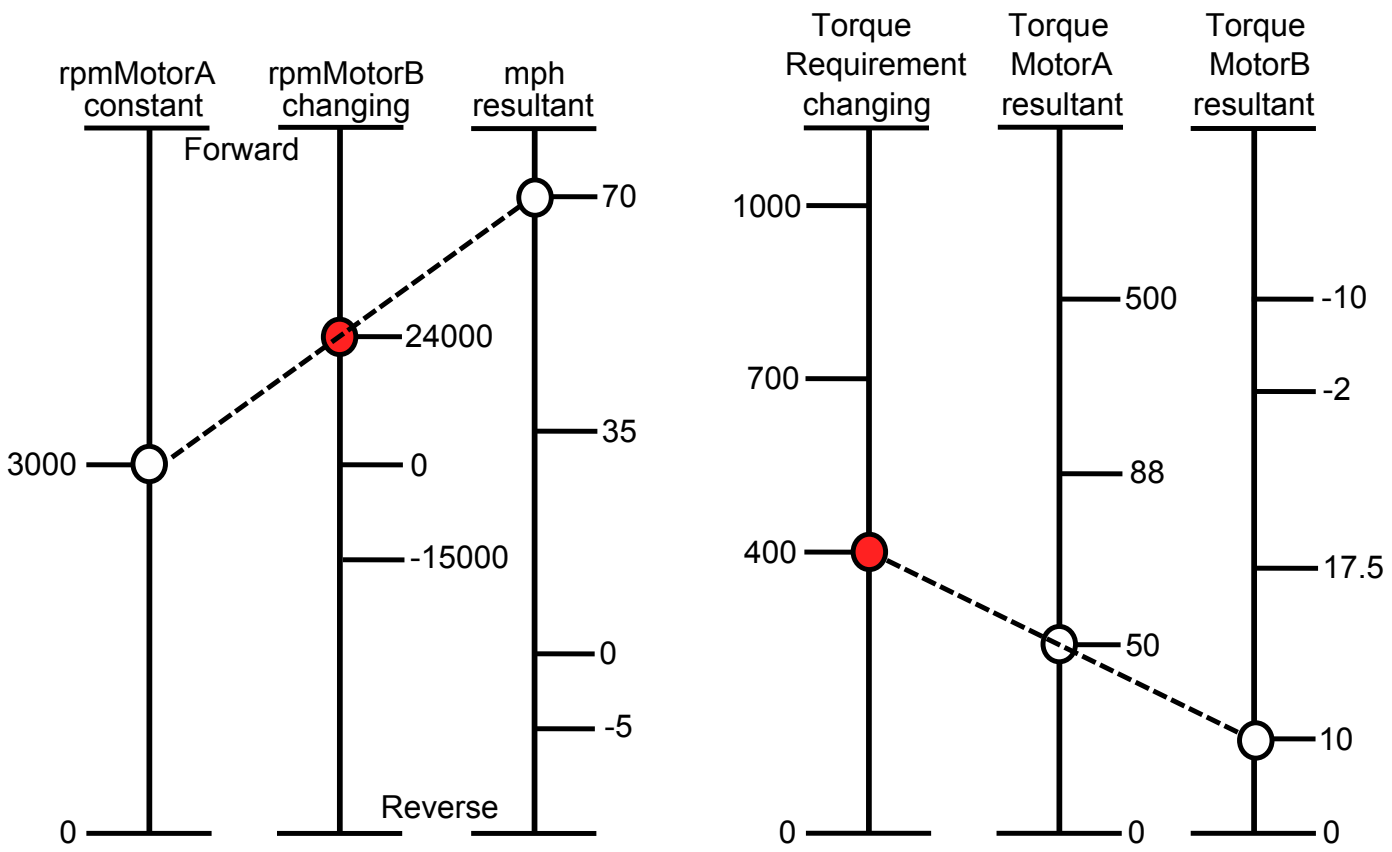

Figure 5.44: Visualization - Vehicle High Speed 

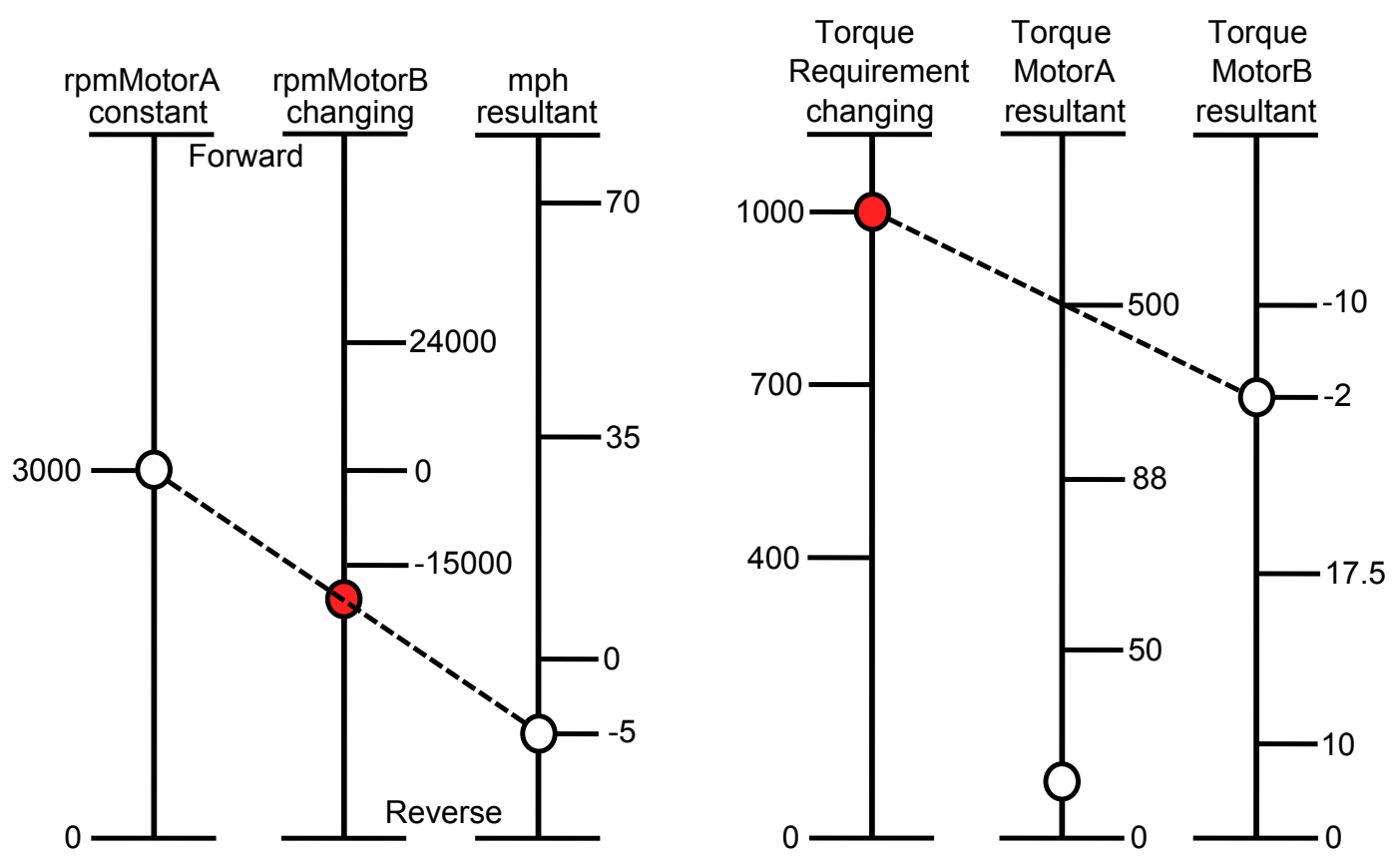

Figure 5.45: Visualization - Vehicle Reverse

Note that in Figure 5.46 the load is shifted to Motor B because Motor B is the input turning in the same direction as the wheels.
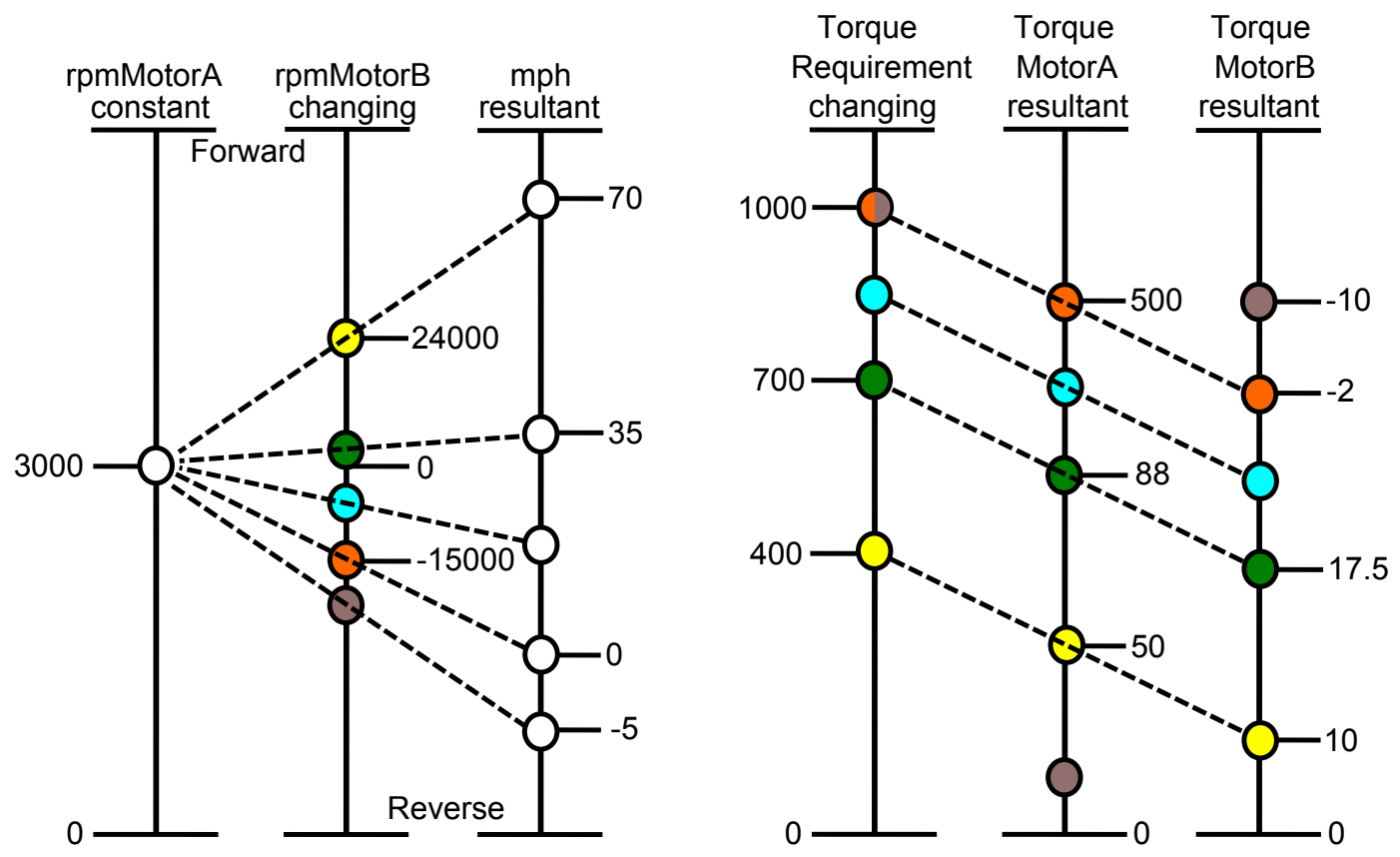

Figure 5.46: Visualization - Torque and RPM Requirements 
Figure 5.46 helps one visualize the complete spectrum of rpm and torque requirements for vehicle performance. In this figure all the previous plots are compiled, each having a unique color to aid in distinguishing between vehicle performance requirements. The colors used on the rpm nomograph correlate directly with those used on the torque nomograph, meaning that the rpm and torque requirements for Motor $\mathrm{A}$ and $\mathrm{B}$ are the same color for a given vehicle performance scenario. The torque requirement of 1,000 is both orange and grey because depending on the vehicle's intended direction (forward or reverse) one motor will be loaded significantly more than the other.

\subsection{System Implications}

\subsubsection{ICE Implications}

The previous example was based on the assumption that Motor A was at a constant rpm. In order for Motor A to achieve a constant rpm when loaded, additional aspects should be considered. If Motor A is an ICE the engine throttle position must be adjusted independently from the vehicle throttle during operation to maintain a constant engine shaft rpm. The necessary adjustment is due to the fact that at a set throttle position an ICE's rpm value will begin to drop as it is loaded. Therefore, a control system or governor to maintain the ICE rpm setting, regardless of loading, should be in place.

\subsubsection{Electric Motor Implications}

If Motor $\mathrm{A}$ is an electric motor the motor construction and operating voltage will contribute largely to the operating rpm. As the electric motor is loaded it will pull more current to meet the instantaneous loading conditions. Results from this research show that, depending on the motor construction, an electric motor can be self-controlling. Having a self-controlled motor for the Motor A input may have significant advantages in maintaining a relatively constant rpm. However an ICE may be required to provide the appropriate torque needed for vehicle propulsion. Further research in this area would coincide well with system optimization. 


\subsubsection{Peak Torque Requirements}

Regardless of what type of input Motor A is, it is important to note the peak torque requirements for both Motor A and Motor B. As discussed previously, peak requirements will be when one input is turning in the positive direction and one is turning in the negative direction. For the previous example, with Motor A as the constant rpm motor and Motor B as the control motor, the peak torque for Motor A would be when the desired vehicle direction is forward from a stopped position. Peak torque for Motor A was illustrated in Figure 5.41. The highest torque requirement for Motor B would be when the vehicle is stopped, but the desired direction is reverse, as in Figure 5.45. It should be noted that it may be possible to keep Motor A as the primary drive motor supporting the load when the desired vehicle direction is reverse by implementing reverse idler gears on both inputs. These peak torque requirements, in conjunction with the required wheel rpm, are the basis for power source selection and gear train optimization.

\subsection{Example Summary}

This example demonstrates the need for a system optimization. In summary, this example illustrates the following:

1. When bevel or spur gears are present, only the input turning in the same direction as the output provides torque to the output

2. When both inputs are turning positively or negatively simultaneously, both provide torque to the output

3. Varying rpm values of Motor B, with Motor A at a relatively constant rpm, governs vehicle speed

4. The torque requirement at the wheels translates back through the gear ratios to the input(s)

5. Changing gear ratios throughout the vehicle layout will provide rpm/torque tradeoffs for system optimization 


\subsection{Results Summary}

Using a differential as a multiple-input, single-output device allows one input to operate at a high efficiency with no needed rpm variation while allowing the other input to vary significantly with respect to its rpm to control output angular velocity.

When used as a multiple-input, single-output device, a differential will split the torque load equally between the multiple inputs if:

1. The inputs are operating in the same direction as the output

2. The gears are of a straight bevel or spur design

3. There is no gear reduction within the system

When the inputs are turning in the same direction the differential will function as a continuously variable transmission (CVT).

In the instance when the inputs are turning in opposite directions, only the input turning in the same direction as the output will provide torque to the output. If the load on the output is large when the inputs are turning in opposite directions, backdrive will occur. To overcome the property of backdrive within the differential, careful consideration should be taken to thoroughly understand torque and rpm performance requirements. Using these performance requirements, an appropriate type of gear set, (i.e. helical, hypoid, worm, etc.) and ratio gear reduction could be used to eliminate backdrive and enable the differential to function as an infinitely variable transmission (IVT) while supporting a load.

Selecting the type of gear reduction (i.e. spur, bevel, helical, hypoid, or worm drive) will have a significant effect on the performance properties of the differential. Specific differential performance properties such as efficiency and backdrivability are affected by gear reduction and type of gear sets used in the differential. 


\section{CHAPTER 6. CONCLUSIONS AND RECOMENDATIONS}

\subsection{Restatement Of Research Objectives}

\subsubsection{Objective 1}

Create a simple graphical model for a dual-input, single-output non-backdrivable differential system.

Objective 1 was accomplished by developing the fundamental theoretical equation, Equation (3.4), and plotting it, as shown in Figure 3.6.

\subsubsection{Objective 2}

Develop theoretical equations for a non-backdrivable differential gear set that relate multiple input torques and rpms to a single output torque and rpm.

Objective 2 was accomplished by developing the theoretical equation, Equation (3.4).

\subsubsection{Objective 3}

Develop a multiple-input, single-output experiment that can prove or disprove the theoretical equations. Reference the experimental data for the multiple-input sources at the applied loads for the performed tests.

The development of a multiple-input, single-output experiment and references to test data was realized as described in Chapters 3 and 4 to meet Objective 3. Fundamental to the success of this research was the original experimental differential which contained ratios of 1:1:1. It was only because of these ratios that the property of backdrive was discovered. If gear reduction had been present in the experimental differential the evidence of backdrive may have been masked and unidentified. 


\subsubsection{Objective 4}

Based upon test results, present recommendations for implementation of a differential as a continuously variable transmission (CVT) or infinitely variable transmission (IVT) in a hybrid vehicle powertrain. Recommendations will be described in the form of cause and effect relationships.

Objective 4 is accomplished hereafter in the following sections of 'Conclusions' and 'Recommendations'.

\subsection{Contributions}

The fundamental contributions of this work are outlined as follows:

1. Identification of backdrive as an important issue

(a) Backdrive occurs when inputs operate in opposite directions and the output is heavily loaded

(b) Backdrive occurs when one input is rotating and one input is stopped and the output is heavily loaded

2. Torque and rpm relationships that exist in a dual-input, single output device

3. Demonstration of a system using a differential as a dual-input, single output device

4. Traction motor and control motor size implications discovered

5. Properties of using a differential as a multiple-input, single-output device

(a) When inputs to a mechanical differential operate in the same direction the differential exhibits CVT characteristics

(b) When inputs to a mechanical differential operate in opposite directions the differential exhibits IVT characteristics 


\subsection{Additional Contributions}

In addition to meeting the research objectives this work has investigated plausible solutions to the challenge of differential backdrive. Also, the investigation of how a differential might be used in a hybrid vehicle powertrain and the implications of such use were discussed. Additional contributions not anticipated for this research include:

1. The fundamental equations that outline differential torque and rpm requirements for use of a differential in a vehicle powertrain as a multiple-input, single-output device

2. The fundamental equations which integrate gear reduction within a differential for vehicle powertrain use as a multiple-input, single-output device

3. The option of a planetary differential as well as the design and optimization benefits of using such a differential

4. An example demonstrating how a differential might be used in a vehicle powertrain application

\section{Implications of control for power sources}

\subsection{Conclusions}

Findings from this research indicate that a differential used as a dual-input, single-output device is a backdrivable torque equalizer. Although torque is equalized between the three input/output elements when they are all rotating in the same direction, rpm values can vary significantly from one I/O to another. Also, one input to a differential can provide the majority of the torque directly while the other input uses gear reduction to provide less torque at increased rpm. When one I/O is loaded a differential that has a 1:1 ratio will split the load between the other two I/Os when the torque supplying I/Os are rotating in the same direction. Some differentials, such as the Gleason-Torsen, have the ability to carry loads unequally on the several I/Os due to high friction coefficients on helical gears.

When spur or bevel gears are used in a differential and the differential inputs are rotating in opposite directions, the input that is rotating in the same direction as the output will be the sole 
provider of torque to the output. In addition to these findings the research results demonstrate that a differential can be used as an infinitely variable transmission while supporting a load. This research also concludes that a planetary differential has probable optimization advantages over conventional differentials and planetary gear sets.

\subsection{Recommendations}

Design, optimize, and build a secondary test setup that includes an appropriately geared planetary differential and conduct testing in a similar manner as has been described in this work to determine appropriate gear types and ratios for using a differential for an IVT application. In conjunction with gear ratio optimization, a vibration analysis should also be conducted to validate the use of a planetary differential as a power transmission device for hybrid vehicle applications. It is anticipated that conducting further experimentation in this manner would yield results in the following areas:

1. The effects of gear ratio reduction on differential backdrive

2. Further refinement of necessary capabilities of inputs (i.e. torque and rpm ranges)

3. System vibration effects

4. Identify potential practical applications for potential use of a mechanical differential as a CVT and/or IVT

5. Control system implications

(a) System control of vehicle

(b) Individual control for system inputs 


\section{REFERENCES}

[1] Granovskii, M., Dincer, I., and Rosen, M. A., 2006. "Economic and environmental comparison of conventional, hybrid, electric and hydrogen fuel cell vehicles." Journal of Power Sources, 159(2), Sept., pp. 1186-1193.

[2] Andersen, B. S., Dalling, R. R., and Todd, R. H., 2008. "A survey of positive engagement, continuously variable transmissions." In 10th International Power Transmission and Gearing Conference, presented at - 2007 ASME International Design Engineering Technical Conferences and Computers and Information in Engineering Conference, IDETC/CIE2007, September 4, 2007 - September 7, 2007, Vol. 7 of 2007 Proceedings of the ASME International Design Engineering Technical Conferences and Computers and Information in Engineering Conference, DETC2007, American Society of Mechanical Engineers, pp. 1081-1087.

[3] Cho, S., Ahn, K., and Lee, J. M., 2006. "Efficiency of the planetary gear hybrid powertrain." Proceedings of the Institution of Mechanical Engineers, Part D: Journal of Automobile Engineering, 220(10), pp. 1445-1454.

[4] Wright, M. T., and Wright, M. T., 2005. "The antikythera mechanism and the early history of the moon phase display." Antiquarian Horology, 29(3 (March 2006)), p. 319329.

[5] http://www.2405.com/press-library/Hummer-H3-2006/hummer-h3-electronic-locking-reardifferential.jpg.

[6] Provatidis, C. G., 2003. http://en.wikipedia.org/wiki/Differential_(mechanical_device).

[7] Dalling, R. R., Haupt, B. L., and Todd, R. H., 2009. "Conceptual design of a positevely engaged continuously variable transmission." In 2008 ASME International Design Engineering Technical Conferences and Computers and Information in Engineering Conference, DETC 2008, August 3, 2008 - August 6, 2008, Vol. 2 of 2008 Proceedings of the ASME International Design Engineering Technical Conferences and Computers and Information in Engineering Conference, DETC 2008, ASME, pp. 955-963.

[8] http://www.richhelms.ca/richhelms/wp-content/uploads/2006/07/s\%20cvt\%20pulleys1.jpg.

[9] http://www.ul.ie/ nolk/gears.htm\#Gear\%20types.

[10] Duoba, M., McGee, R., and Theobald, M. A., 2004. Advanced Hybrid Vehicle Powertrains 2004: Sp-1833. Society of Automotive Engineers Inc, Mar.

[11] Edwards, J. R., 1991. United states patent: 5067932 - dual-input infinite-speed integral motor and transmission device, Nov.

[12] Edwards, J., and Tether, D., 1996. United states patent: 5575730 - multiple-input infinitespeed integral motor and transmission device, Nov. 
[13] Yamaguchi, K., and Miyaishi, Y., 1997. United states patent: 5643119 - hybrid vehicle powertrain, July.

[14] Yamamoto, Y., and Chubachi, K., 1998. United states patent: 5755303 - power transmitting apparatus for a hybrid vehicle, May.

[15] Brown, L. T., Ortmann, W. J., and Kraska, M. P., 2001. United states patent: 6176808 hybrid vehicle powertrain and control therefor, Jan.

[16] Pesiridis, R. A., and Christian, A. J., 2009. United states patent: 7481730 - multiple input, dual output electric differential motor transmission system, Jan.

[17] http://john1701a.com/prius/prius-energy.htm.

[18] http://en.wikipedia.org/wiki/File:Epicyclic_gear_ratios.png.

[19] Ahn, K., Cho, S., Cha, S. W., and Lee, J. M., 2006. "Engine operation for the planetary gear hybrid powertrain." Proceedings of the Institution of Mechanical Engineers, Part D: Journal of Automobile Engineering, 220(12), pp. 1727-1735 Compendex.

[20] http://en.wikipedia.org/wiki/Toyota_Prius.

[21] http://www.toyota.com/prius-hybrid/trims-prices.html.

[22] http://en.wikipedia.org/wiki/Chevrolet_Volt.

[23] http://www.gm.com/experience/technology/news/2008/volt_092908.jsp.

[24] http://gm-volt.com/2010/10/11/motor-trend-explains-the-volts-powertrain/.

[25] http://members.rennlist.com/951_racerx/PS84_Diff1.gif.

[26] Benjamin C. Groen, ., 2010. Investigation of dc motors for electric and hybrid electric motor vehicle applications with an infinitely variable transmission.

[27] Budynas, R. G., and Nisbett, J. K., 2008. Shigley's Mechanical Engineering Design., eighth ed. McGraw-Hill.

[28] Benford, H. L., and Leising, M. B., 1981. "Lever analogy: A new tool in transmission analysis." SAE Preprints(810102) Compendex. 


\section{APPENDIX A.}

\section{A.1 Experimental Setup}

\section{A.1.1 General Layout}

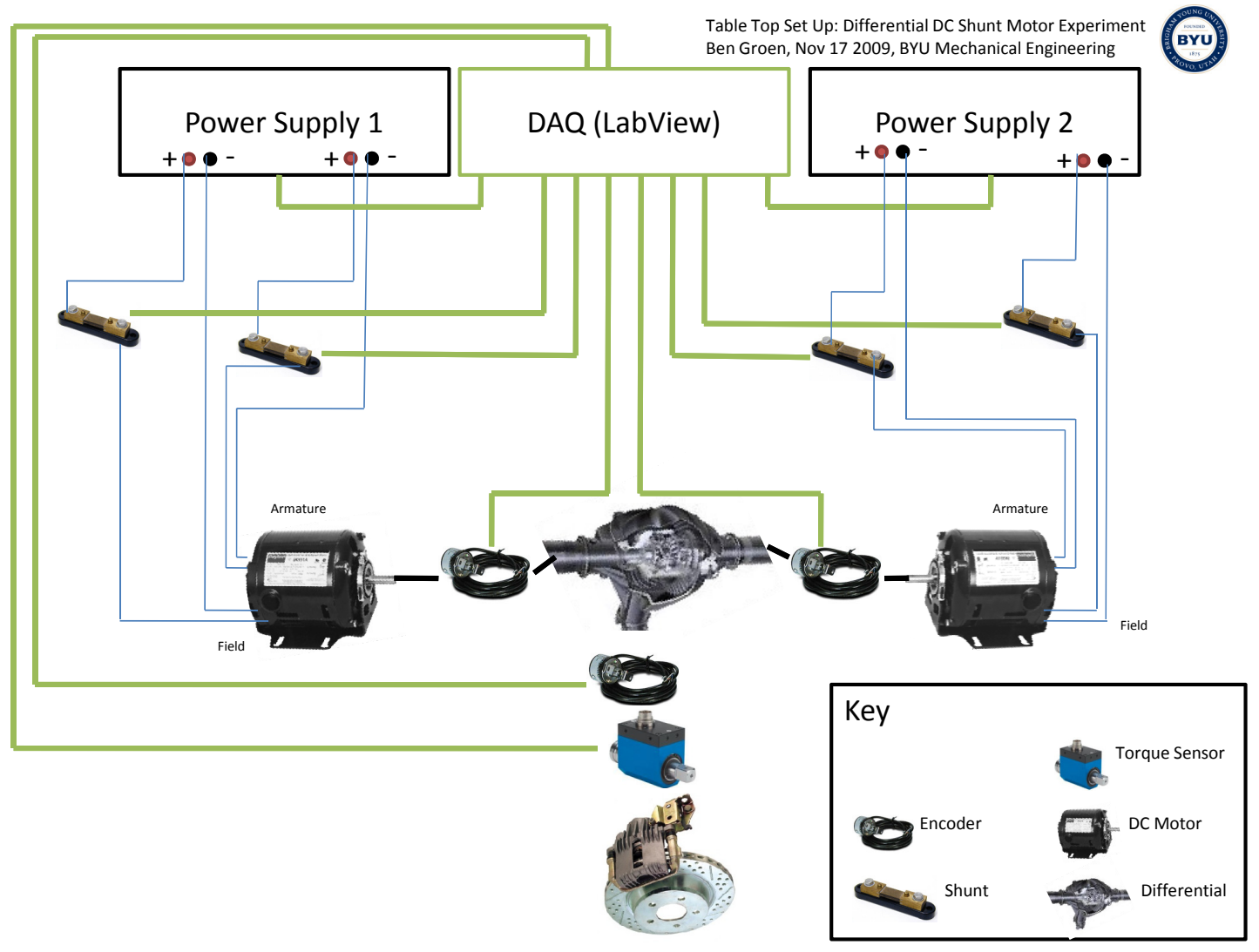

Figure A.1: Setup Diagram 


\section{A.1.2 Detailed Experimental Wiring Diagram}

\section{CVT Differential Motor Testing Schematic}

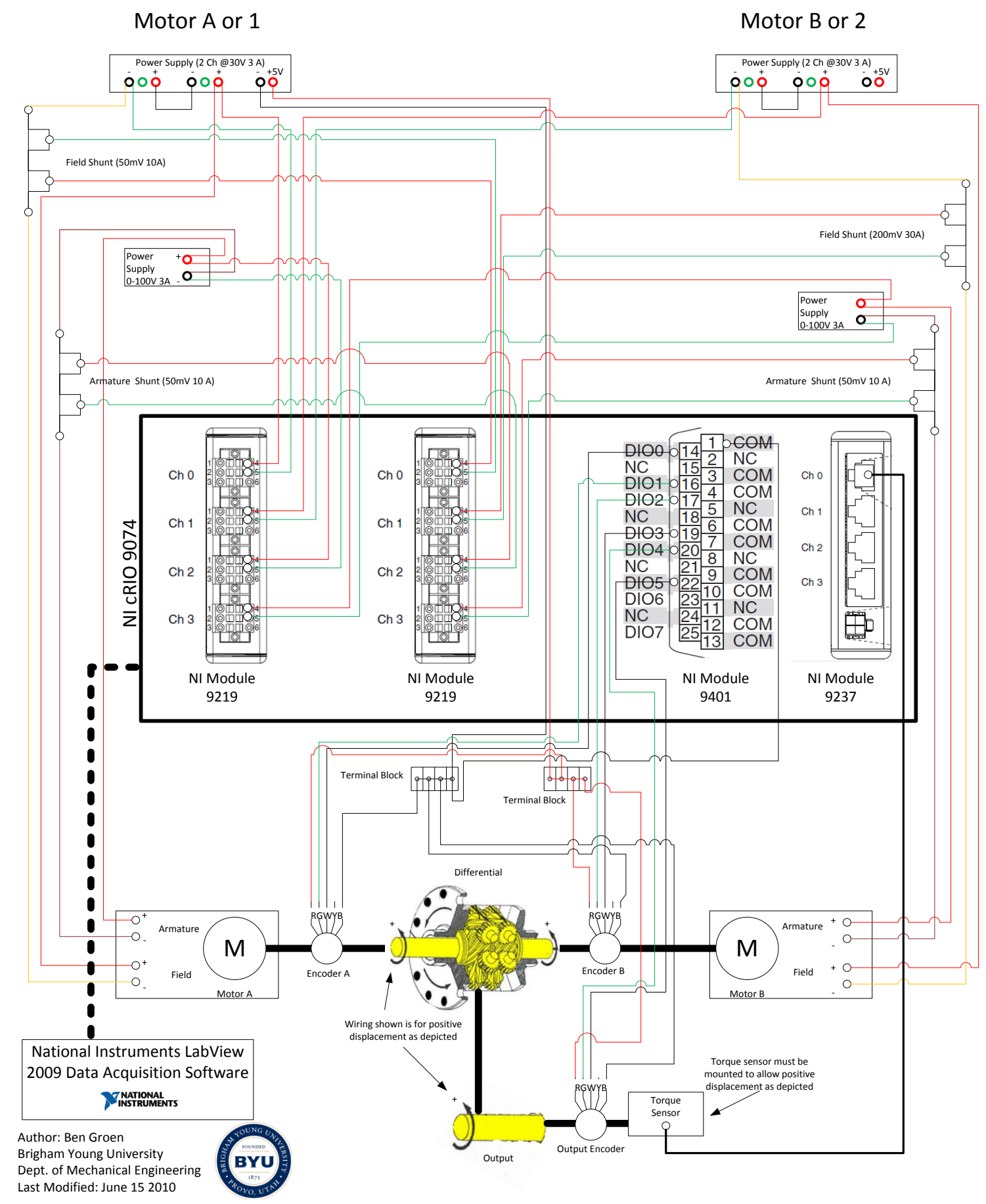

Figure A.2: Wiring Schematic 


\section{A.2 Electric Motor Characterization}

\section{A.2.1 No Load Speed Test - Varying Field Voltage}

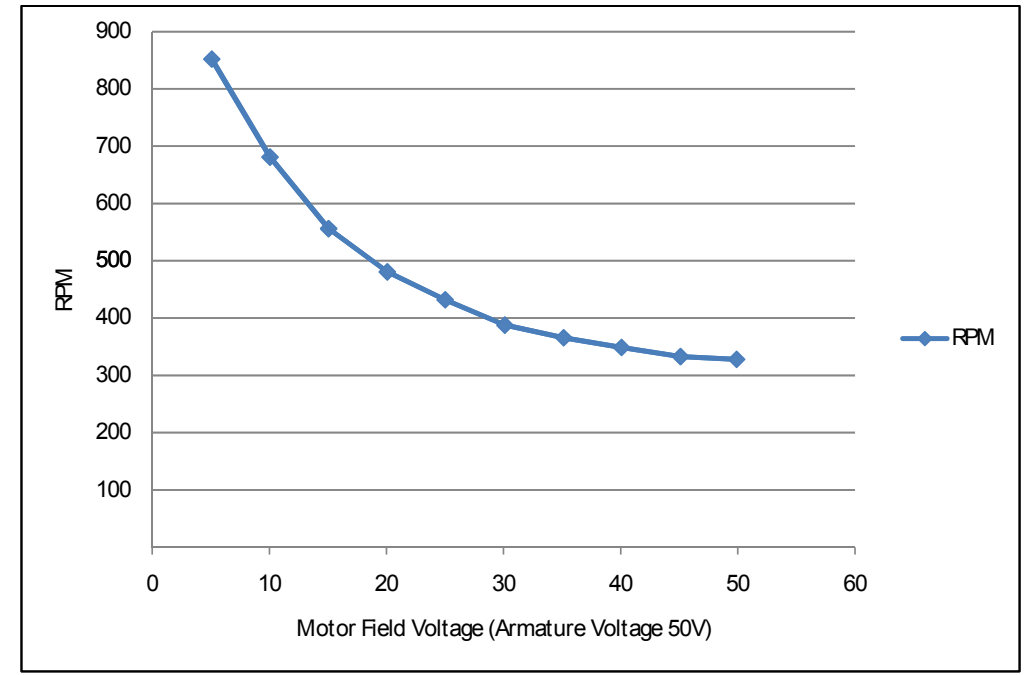

Figure A.3: Field Variation

\section{A.2.2 No Load Speed Test - Varying Armature Voltage}

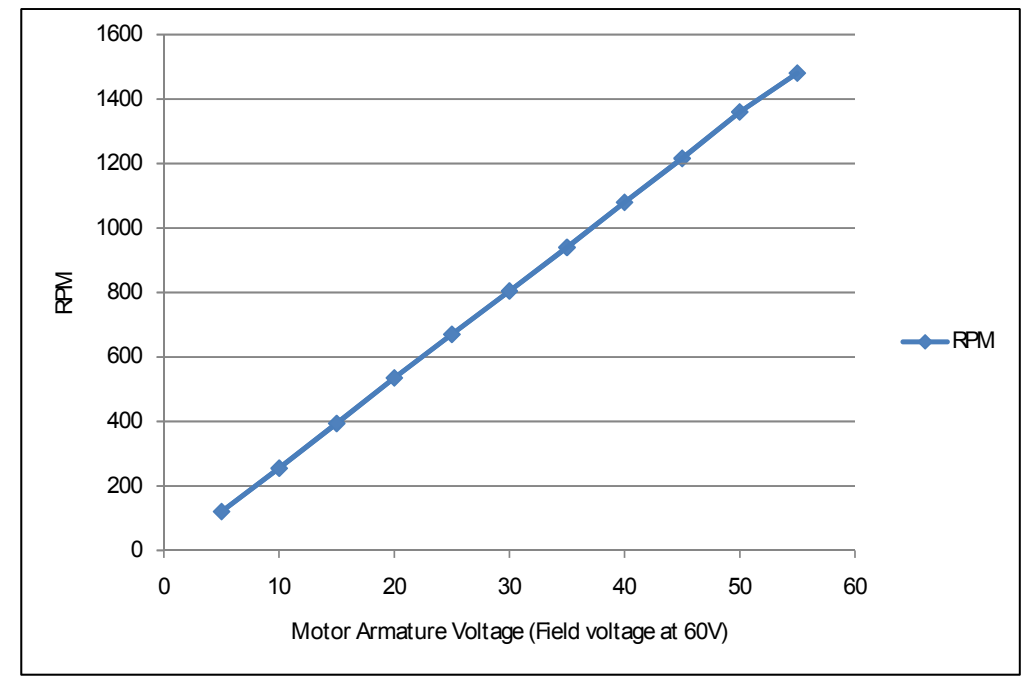

Figure A.4: Armature Variation 


\section{A.2.3 Load Test}

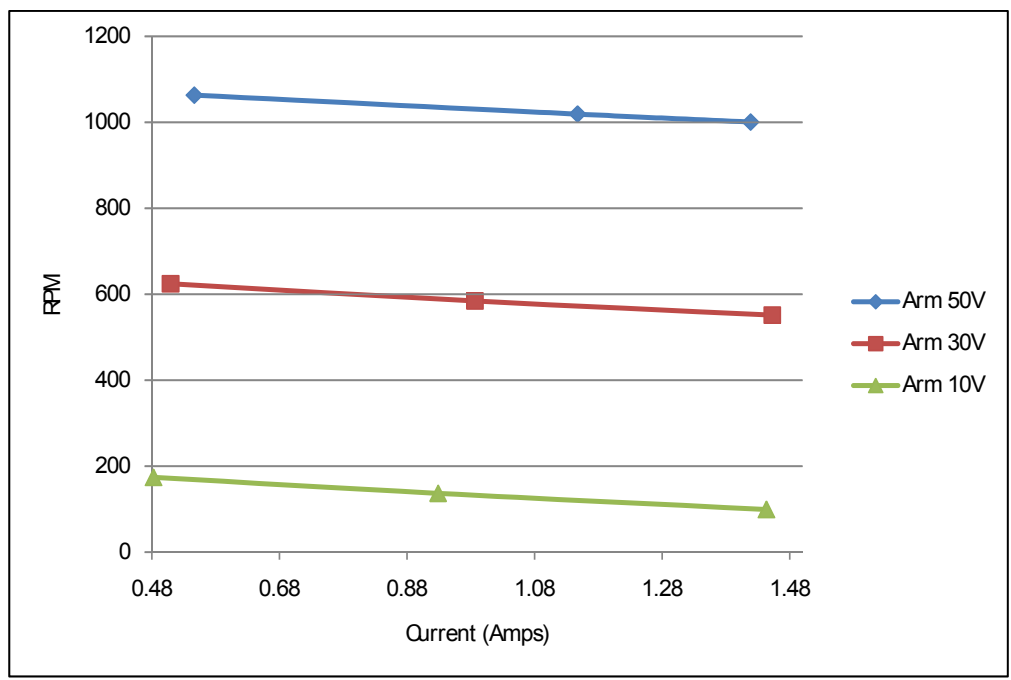

Figure A.5: Electic Motor RPM Characterization

\section{A.2.4 Load Test - Field And Armature Trends}

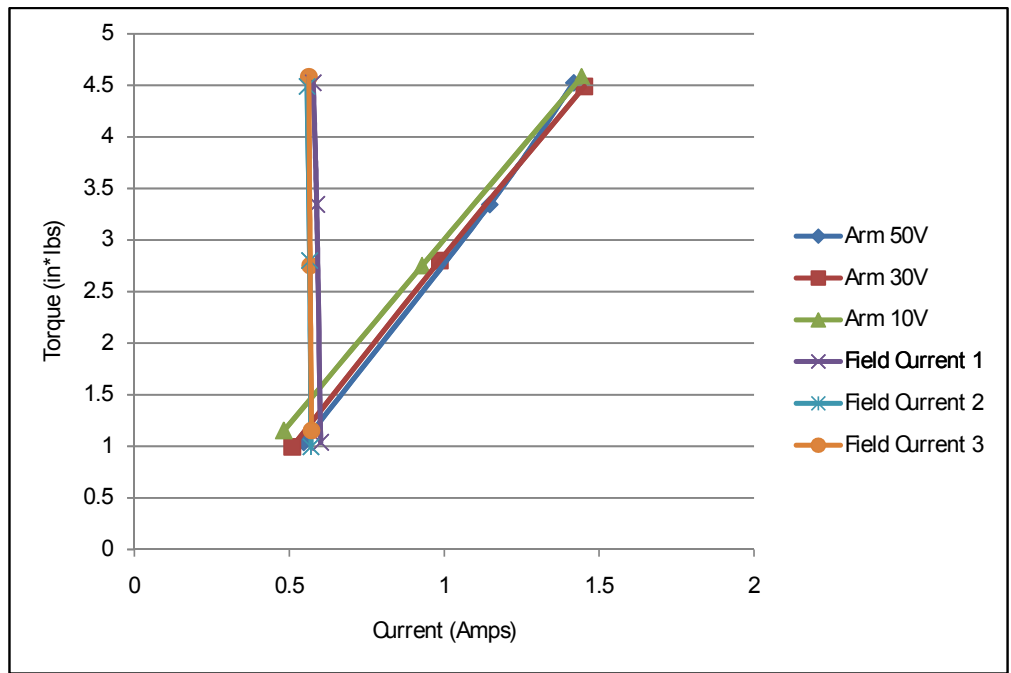

Figure A.6: Electic Motor Torque Characterization

Figures A.5 and A.6 show that regardless of rpm it is the current to the armature that dictates the output torque of the motor. 


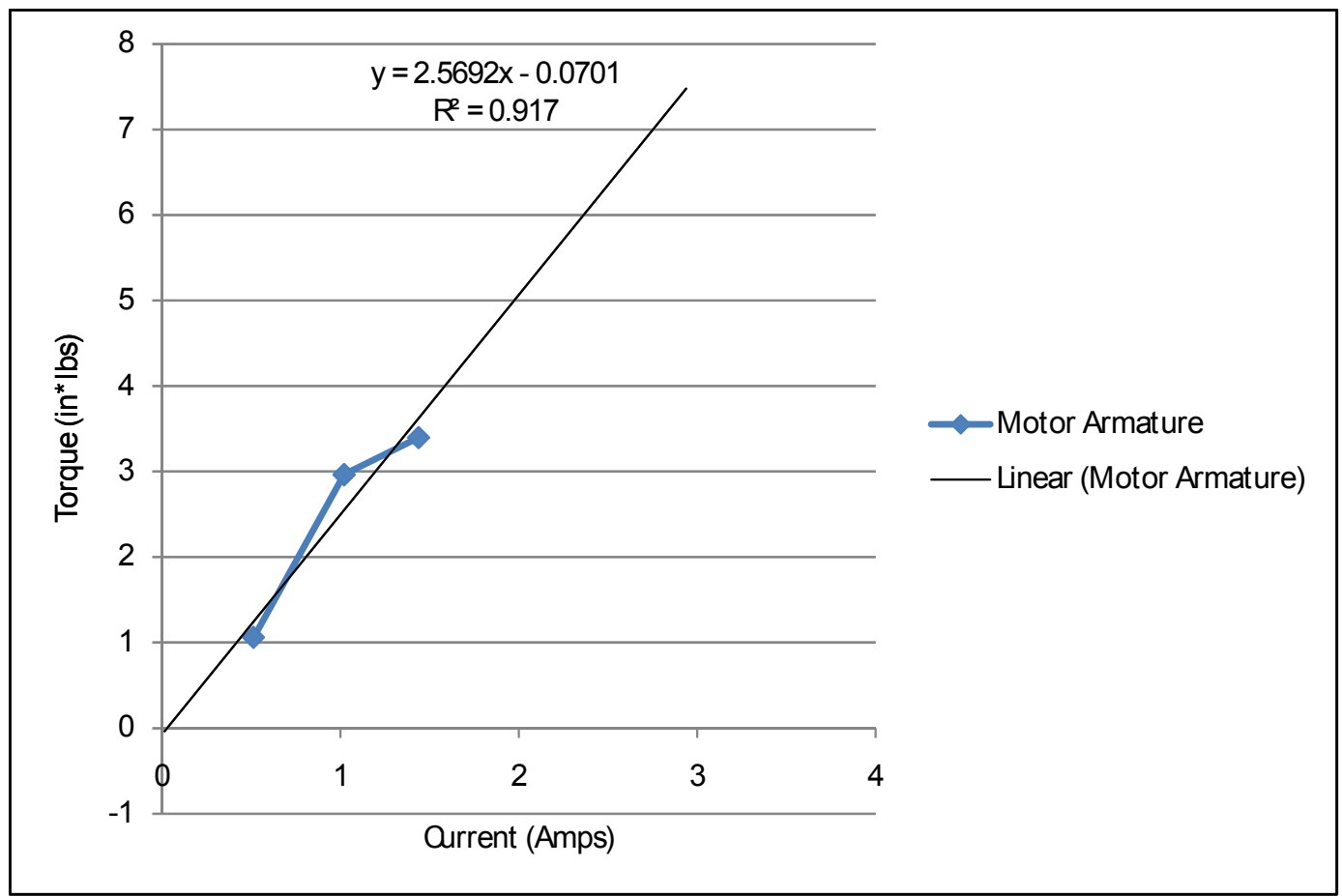

Figure A.7: Electic Motor Torque Characterization: Trend Line

Figure A.7 shows the trend line used to correlate current to the armature with motor torque for subsequent tests. 